\title{
Determinants and outcomes of group coordination and decision-making in red-fronted lemurs (Eulemur rufifrons)
}

\author{
Dissertation \\ zur Erlangung des mathematisch-naturwissenschaftlichen Doktorgrades \\ „Doctor rerum naturalium" \\ der Georg-August-Universität Göttingen
}

vorgelegt von

Lennart W. Pyritz

aus

Bielefeld

Göttingen 2011 

Referent: Prof. Dr. Peter M. Kappeler Korreferent: Prof. Dr. Eckhard W. Heymann

Tag der mündlichen Prüfung: 30. Juni 2011 



\section{PREFACE}

Chapter 1 of this thesis comprises three articles (Chapter 1.1-1.3) that deal with similar issues. Each of the other chapters comprises one article. The majority of papers presented in this thesis (Chapter 1.1-1.3, Chapter 3 and Chapter 5) have been accepted by different scientific journals, all of them peer-reviewed except for a book chapter (Chapter 1.1) and a short communication (Chapter 5). Chapters 2 and 4 are currently under revision. Chapters 1.1, 1.2 and 5 have already been published. The two other accepted articles will be published later this year in a special issue on group coordination and decision-making of the International Journal of Primatology that is based on a symposium at the XXIII Congress of the International Primatological Society in Kyoto, Japan, September 2010. The symposium was organised by Andrew J. King and Cédric Sueur with whom I collaborated on a paper on terminology and concepts used in coordination and decision-making research as a consequence of discussions during the congress (Chapter 1.3). I collected most of the field data presented in this thesis in cooperation with Jean-Pierre Tolojanahary, a Malagasy field assistant at the research station of the German Primate Center, who also continued data collection when I was not in Madagascar. Data presented in Chapter 4 were collected with the help of three additional Malagasy assistants: Patrick de Beroboka, Rémy d’Ampataka and Tianasoa Andrianjanahary. With the latter I also observed the collective anti-predator behaviour described in Chapter 5. Claudia Fichtel and Peter Kappeler developed the project idea and supervised the thesis in the field and in Göttingen and are co-authors on the other articles. Claudia Fichtel is first author of the book chapter (Chapter 1.1) because it is based on a presentation she gave at the workshop "Assessment methods of coordination processes" in Reinhausen, December 2007. Elise Huchard contributed importantly to data analyses in Chapter 4. The chapters of this work correspond to the published or submitted articles with one exception. In the original versions of Chapters 1.1, 1.2 and 5 the old scientific name of red-fronted lemurs is used (Eulemur fulvus rufus), which I changed to the new name (Eulemur rufifrons: Mittermeier et al. 2008) in this thesis. 


\section{CONTENTS}

GENERAL INTRODUCTION

\section{ChAPTER 1: ReVIEW, CONCEPTS AND TERMINOLOGY}

1.1 Coordination of group movements in non-human primates

with Claudia Fichtel and Peter M. Kappeler

in Boos M, Kolbe M, Kappeler PM, Ellwart T (eds) Coordination in human and primate groups. Springer, Heidelberg, pp 37-56 (2011)

1.2 Conceptual and methodological issues in the comparative study of collective group movements

with Claudia Fichtel and Peter M. Kappeler.

Behavioural Processes 84: 681-684 (2010)

1.3 Reaching a consensus: terminology and concepts used in coordination and decisionmaking research

with Andrew J. King, Cédric Sueur and Claudia Fichtel.

International Journal of Primatology, Special Issue: Group Coordination \& DecisionMaking, in press (2011)

\section{Chapter 2: LARge-SCAle RANGing PATterns}

Data from GPS collars highlight effects of sampling and calculation method, climatic seasonality and sample size on home range estimates in a wild primate

With Claudia Fichtel and Peter M. Kappeler. .55

International Journal of Primatology: submitted

\section{Chapter 3: GROUp CoORdination under Natural Conditions}

Coordination of group movements in wild red-fronted lemurs: Processes and influence of ecological and reproductive seasonality

with Peter M. Kappeler and Claudia Fichtel...... .75

International Journal of Primatology, Special Issue: Group Coordination \& DecisionMaking, accepted with minor revisions (2011) 
Chapter 4: Group CoORdinAtion duRING A Foraging Experiment

Determinants and outcomes of decision-making, group coordination and social interactions during a foraging experiment in a wild primate

with Claudia Fichtel, Elise Huchard and Peter M. Kappeler.. 103

Behavioral Ecology and Sociobiology: submitted

Chapter 5: Collective Anti-Predator Behaviour

Collective mobbing of a boa by a group of red-fronted lemurs (Eulemur rufifrons)

with Tianasoa Andrianjanahary.

Lemur News 15:15-17 (2010)

GENERAL DisCUSSION

SUMMARY

ZUSAMMENFASSUNG.

REFERENCES

APPENDIX 


\section{GENERAL INTRODUCTION}

This thesis investigates conceptual and empirical questions regarding coordination and decision-making in the context of (primate) group movements. In this general introduction, I will provide a short overview of the costs, benefits and inter-individual conflicts that mould sociality and require coordination and collective decisions in animal groups in the first place. Subsequently, I will outline why group movements emerged as the major paradigm in studies on group coordination and decision-making, how they can be analysed systematically and why primates provide an excellent model taxon. Next, I will briefly address a number of conceptual and terminological problems that have hampered a broader integration of coordination studies in the past. Finally, I will highlight which conceptual and empirical aspects of coordination and decision-making I explored in the single chapters of this thesis and which approaches I used to do so.

\section{Why do animal groups need coordination and decision-making?}

Maynard Smith and Szathmáry (1995) rated the shift from a solitary lifestyle to colonies, i.e. individuals living in permanent groups with regular social interactions, as one of the eight major transitions in evolution. Sociality is a universal feature in the animal kingdom and an impressive variability in group characteristics in terms of size, social structure, (temporal) cohesion, permanence and species composition within groups can be found. A large body of literature exists that explores the evolutionary pathways towards sociality, i.e. the trade-offs between costs and benefits of grouping (see Krause and Ruxton 2002 for an overview). In most species, reduced per capita predation risk seems to be the major selective force favouring group-living through shared vigilance, predator confusion or collective anti-predator behaviour (Alexander 1974; van Schaik 1983; Caro 2005). Further species-specific benefits include joint defence of a territory or foraging patches, cooperative hunting, communal care of offspring and enhanced thermoregulation (Wrangham 1980; Brown 1987; Goldizen 1987; Boesch 1994; Scantlebury et al. 2006). However, group-living also entails costs due to inter-individual conflicts among group members. These costs include intra-group competition over resources and mating opportunities as well as facilitated transmission of pathogens (Bertram 1978; van Schaik 1989; Altizer et al. 2003). Conflicts within a group are also expected to arise from 
differences among individuals in terms of physiological constitution - promoting different activity budgets and foraging patterns - or differing mating strategies among females and males (Bercovitch 1983; Fischhoff et al. 2007).

Despite these costs and conflicts, group-living animals have to stay in close spatial proximity and synchronise their activity patterns to reap the benefits of sociality, requiring coordination and collective decisions of group members (Rands et al. 2003; Conradt and Roper 2005). In simple scenarios, individual interests overlap and external stimuli can synchronise individual decisions, automatically resulting in a collective behaviour of group members. Coordination can also be achieved by self-organisation based on local communication, i.e. individuals follow simple behavioural rules and copy the behaviour of their next neighbours without the need for global control (Camazine et al. 2001; Couzin et al. 2005; Sumpter 2006). However, if individual interests diverge and a decision has to be made between two or more mutually exclusive actions, consensus decisions are required that usually involve more complex mechanisms, such as leadership, negotiation and signalling behaviour (King and Cowlishaw 2009; Kerth 2010a; Fischer and Zinner 2011). Consensus decisions can be (partially) shared with several or all individuals contributing to the decision outcome, or unshared, i.e. one individual decides without considering preferences or behavioural responses of other group members (Conradt and Roper 2005, 2007).

In general, fitness consequences of consensus decisions do not only relate to group cohesiveness and mediation of consensus costs among group members, but also comprise information pooling, and speed/accuracy of decisions (Seeley and Buhrman 2001; Franks et al. 2002; Conradt and Roper 2003). For instance, Ward et al. (2011) showed that larger shoals of mosquitofish (Gambusia holbrooki) made faster and more accurate decisions regarding the avoidance of a predator model than smaller shoals, a phenomenon called "wisdom of the crowd" (Conradt 2011).

\section{Why and how to study group movements?}

Most recent theoretical (Rands et al. 2003; Couzin et al. 2005) and empirical studies (Stueckle and Zinner 2008; Ramseyer et al 2008a, b, c; Bourjade et al. 2009; Jacobs et al. 2011) have focused on collective movements to explore coordination and decisionmaking in animal groups (including humans: Dyer et al. 2008). In fact, group movements 
provide an outstanding model in this context (see Kappeler 2011 for a summary). First, they occur regularly because most group-living species have to search for food, travel between suitable resting sites, avoid predator encounters or patrol territorial borders on a daily basis. Second, group movements provide an ecologically highly significant context during which group members have to synchronise and coordinate activity schedules despite different physiological needs (Rands et al. 2003; Petit and Bon 2010). Previous studies were short-termed and focused on single aspects of coordination during group movements, such as initiation signals and travel calls, leadership in specific situations or mechanisms mediating group decisions (Boinski 1991; Bonanni et al. 2010; Bousquet et al. 2010; Sueur et al. 2010). However, group movements rely on a number of interacting behavioural processes (Trillmich et al. 2004) and are characterised by dynamics operating at multiple levels, each of which provides valuable information to understand how animal groups coordinate during collective movements.

\subsection{Spatio-temporal patterns}

The most basic way to explore group movements is to analyse variability of spatiotemporal ranging patterns of a group in its natural habitat throughout different socioecological conditions. Numerous studies showed that ranging behaviour is influenced by the availability and distribution of resources, climatic seasonality or inter-group encounters (e.g., snub-nosed monkeys, Rhinopithecus roxellana: Li et al. 2000; lions, Panthera leo: Spong 2002; meerkats, Suricata suricatta: Furrer et al. 2011). In turn, decreasing resource abundance can lead to increased intra-group competition (Koenig 2002), or inter-group encounters can yield different costs or benefits for females and males (Fashing 2001), resulting in inter-individual conflicts that affect coordination processes and decision-making. Analyses of spatio-temporal ranging patterns can therefore help to identify the reasons underlying context-dependent variation in the coordination of group movements.

\subsection{Processes and leadership}

The next step relates to the analysis of the basic behavioural processes underlying coordinated group movements, i.e. which individuals initiate, lead and terminate a movement, and how many group members follow whom in which time frame (Trillmich 
et al. 2004). Movements of large, anonymous groups are regulated by local communication and often self-coordinated by simple behavioural rules (honey bees, Apis mellifera: Seeley and Buhrmann 2001; overview on vertebrates: Parrish et al. 2002; Couzin and Krause 2003; but see Conradt et al. 2009). In contrast, small, non-anonymous groups are usually characterised by global communication and leadership by specific individuals (green woodhoopoes; Phoeniculus purpureus: Radford 2004; harem groups of plains zebras, Equus burchellii: Fischhoff et al. 2007; dogs, Canis lupus familiaris: Bonanni et al. 2010; bottlenose dolphins, Tursiops truncatus: Lewis et al. 2011).

In addition, recruitment success of leaders can be judged with respect to individual characteristics, such as dominance rank, social connectivity, motivation, knowledge and personality (see King et al. 2009 for a summary). In fact, several of these characteristics may coincide within an individual (e.g., dominance and strong affiliative network relations: King et al. 2008) or different individuals may be motivated most or informed best depending on the context of the collective action (state-dependent leadership: Rands et al. 2008). For instance, in chacma baboons (Papio ursinus) consistent leadership of the dominant male of the group was observed during movements to artificial foraging patches (King et al. 2008), whereas Stueckle and Zinner (2008) reported distributed leadership of males and females during morning departures from a sleeping site in the same species. In summary, the analysis of coordination processes in a natural habitat is indispensable to identify species-specific socio-ecological determinants of leadership and followership.

\subsection{Mechanisms of group coordination}

Initiators of group movements may use a variety of visual and acoustic signals to recruit followers including intention movements, back-glances or travel calls (e.g., swans, Cygnus cygnus and Cygnus columbianus bewickii: Black 1988; African elephants, Loxodonta africana: Poole 1988; primates: Leca et al. 2003; Meunier et al. 2008; Sueur and Petit 2010). In addition, a number of mechanisms, such as voting and mimetism (e.g., Sueur et al. 2010), can govern follower behaviour during departure. Voting in the form of a quorum response has been described in self-organised systems with local communication (nest choice in honey bees: Seeley and Buhrman 2001; house-hunting in ants: Franks et al. 2003), but also in small groups with global communication (hamadryas baboons, Papio 
hamadryas: Stolba 1979; white-faced capuchins, Cebus capucinus: Petit et al. 2009; meerkats: Bousquet et al. 2010; Tonkean macaques, Macaca tonkeana: Sueur and Petit 2008a; Sueur et al. 2010).

Another mechanism mediating follower behaviour is mimetism or amplification, i.e. the probability of an individual taking part in a collective action depends on the number of group members already performing this behaviour (Camazine et al. 2001; Sumpter 2006). If the identity of group members does not influence the joining probability of an individual, mimetism is anonymous (white-faced capuchin monkeys: Meunier et al. 2006). On the other hand, an individual's decision to join may depend on the behaviour of its preferred social partners or kin (selective mimetism: Sueur et al. 2009). Joining according to affiliative relationships has been described in captive groups of Tonkean macaques (Sueur et al. 2010) and brown lemurs (Eulemur fulvus fulvus: Jacobs et al. 2011). Mimetism and quorum decisions are not mutually exclusive but may occur simultaneously during initiation of group movements (Petit et al. 2009; Sueur et al. 2010). Exploring these mechanisms is crucial to understanding how coordination processes are proximately controlled. A comparison of coordination mechanisms among different species, ranging from bees to primates, also illuminates the impact of cognitive abilities on group coordination (Sueur and Deneubourg 2011).

\subsection{Decision types}

Finally, the mechanisms mediating group coordination determine the type of decisionmaking during a collective action. As stated earlier, consensus decisions can either be (partially) shared or unshared, depending on the number of group members contributing to the decision outcome (Conradt and Roper 2005). Based on this definition, decisions via a quorum are (partially) shared because a subset of or all group members vote for a specific action in order to reach a consensus (Sueur and Petit 2008a, b). The classification of mimetism in the framework of consensus decisions is more difficult. One could argue that mimetism is a self-organised mechanism that amplifies automatically and, hence, group members do not need to achieve a consensus actively (Sueur and Deneubourg 2011). However, several or all group members contribute significantly to the decision outcome when mimetism is involved. From this perspective, the decisions mediated by mimetism are (equally) shared. 
Conradt and Roper (2007) argued that completely unshared decision-making is less likely to evolve than shared decision-making due to high consensus costs for followers. In fact, it has been described for only a few species based on observations of consistent leadership by dominant individuals in specific contexts (wolves, Canis lupus: Mech 1970; dwarf mongooses, Helogale parvula: Rasa 1987; mountain gorillas, Gorilla beringei beringei: Watts 2000; chacma baboons: King et al. 2008 and Guy Cowlishaw pers. comm.). However, observations of follower behaviour in mountain gorillas revealed an increase in grunt rates by several group members just prior to a coordinated group departure (Stewart and Harcourt 1994). Thus, apparently not only the dominant individual decided when to move off, but the other group members also participated in the decision by signaling their readiness to depart in a vocal quorum. Similar mechanisms may also be in place in wolves, mongooses and baboons but systematic studies have not been conducted yet (but see Fischer and Zinner 2011 for preliminary results in baboons).

\section{Why study primates?}

Primates provide an excellent model to conduct comparative studies on group coordination for several reasons. First, most primates are group-living, and their groups comprise individuals of different age and sex classes that are connected in complex social networks with varying rank systems (Dunbar 1988; Mitani et al., in press). These characteristics can be expected to promote inter-individual conflicts of interest, challenge group cohesion and, thus, require coordination of collective activities in the first place. Furthermore, primate habitats differ enormously in terms of resource abundance, climatic seasonality and predation risk (Wolfheim 1983). Such pronounced variability allows exploring diverse ecological determinants of group coordination and decisionmaking. Finally, primates are also a convenient model due to their high cognitive abilities and complex communication (Reader and Laland 2002; Barrett and Henzi 2005; Tomasello and Zuberbühler 2002) - traits that (theoretically) enable them to make sophisticated decisions and coordinate collective activities using a wide spectrum of different mechanisms. 


\section{Conceptual and methodological problems}

The small collection of studies presented in this general introduction indicates that processes and mechanisms involved in the coordination of group movements are complex, and that researchers have approached the problem with various methods. In fact, it is not possible to integrate all studies on group coordination into one of the existing frameworks because they either focus on the mode of communication and the degree of conflict among group members, on determinants of leadership or on mechanisms of decision-making during different stages of group departure, and they only partly overlap (Conradt and Roper 2005; Conradt and List 2009; King et al. 2009; Bourjade and Sueur 2010). Furthermore, terminology and concepts are not always used consistently, especially comparing studies on coordination in animals and humans (see Dumont et al. 2005; Fischer and Zinner 2011 for further discussion). Therefore, I will also address a number of conceptual and terminological issues in this thesis.

\section{Aims and approaches of this thesis}

The overall aim of this thesis is twofold: As indicated above, I critically review current concepts, methods and terminology used in coordination research and highlight a number of concrete suggestions that could improve comparability among future studies and facilitate the identification of coordination patterns shared among different species. In applying these standards, I provide a comprehensive analysis of collective movements in groups of wild red-fronted lemurs (Eulemur rufifrons) by exploring (i) spatio-temporal patterns of ranging behaviour; (ii) coordination processes of group movements across different ecological and reproductive seasons; and (iii) coordination of group movements and decision outcomes during a foraging experiment with artificially altered conflict potential. Coordination mechanisms - the fourth level characterising coordination of collective movements - were studied simultaneously as part of a diploma thesis (Pflüger 2010) that I will address briefly in the general discussion.

Red-fronted lemurs are a convenient study species due to a number of reasons. (1) Most research on coordination in small animal groups dealt with species living in hierarchically organised groups and revealed strong effects of dominance rank on leadership (Boinski 1993; King et al. 2008; Bonanni et al. 2010; Šárová et al. 2010). In contrast, red-fronted lemurs live in small, multi-male-multi-female groups characterised 
by an egalitarian social structure (Kappeler 1991; Pereira and McGlynn 1997), which offers the opportunity to test effects of alternative determinants of leadership. (2) Our study groups reproduce seasonally, reside in a habitat with pronounced ecological seasonality and experience veritable predation risk (Barthold et al. 2009; Sorg and Rohner 1996; Rasoloarison et al. 1995). All of these factors can possibly affect coordination processes and, thus, help to identify crucial determinants of leadership and decisionmaking (Kerth 2010a). (3) Finally, lemurs provide comparative data on the evolution of group coordination in primates compared to anthropoid taxa due to their unique evolutionary history (Kappeler 1999).

Chapter 1.1 starts with a review of the literature on coordination of group movements in primates structured according to the four different levels outlined above. The focus then shifts to methodological issues, and I discuss definitions of group movements used in previous studies and propose taxon-specific definitions based on empirical data. Finally, I briefly discuss to which extent studies on groups of non-human primates are transferable to human groups and vice versa. This last issue is included because the chapter is published in a book that examines coordination in human and nonhuman primates in an interdisciplinary approach. Chapter $\mathbf{1 . 2}$ is a commentary that critically discusses comparability of data on coordination collected in captive and freeranging groups, respectively. Here, I further discuss the limitations of transferring concepts from game theoretical and agent-based models to empirical contexts as a result of the importance of variables such as "assertiveness" that usually cannot be assessed in real animals. Then, I introduce a taxon-specific approach to operationalise group movements in red-fronted lemurs by presenting data from a pilot study and highlight the future importance of field experiments. In Chapter 1.3, I identify a number of ambiguous terms and conceptual problems in coordination research and propose ways to improve comparability among future studies.

Chapter 2 elaborates on the analysis of spatio-temporal ranging patterns in primates and describes a purely methodological approach: I illustrate the effects of sampling method, analytical method, climatic seasonality and sample size on home range estimates in red-fronted lemurs in order to highlight the influence of these variables on the comparability among studies. 
The following chapters deal with behavioural data collected during field work in Madagascar. In Chapter 3, I analyse coordination processes in four groups of red-fronted lemurs throughout a whole year in order to explore consequences of ecological and reproductive seasonality on leadership and follower behaviour. Chapter 4 presents results from a foraging experiment with artificial drinking platforms that was designed to create decision conflicts within a group by providing either one individual with a large amount or several group members with smaller amounts of a high-value resource on different platforms. Given varying degrees of conflict among group members through different baiting patterns, I examine determinants of decision-making, coordination, group fission and aggressive behaviour at the foraging platforms and outcomes in terms of individual resource intake.

Chapter 5 reports a case of collective mobbing of a predator by a group of redfronted lemurs that I observed by chance at Kirindy Forest. I decided to include this short communication in my thesis because it illustrates the fact that collective behaviour does not only play a role during group movements, but that it is required in various behavioural contexts, including conflict resolution and collective vigilance (Aureli et al. 2002; Sirot and Touzalin 2009).

Finally, I summarise the major results of the thesis, discuss them in relation to findings from other studies, and provide an outlook on future research needed to complement our understanding of coordination and decision-making in animal groups. 
CHAPTER 1.1

COORdinAtion of GRoup MOVEMENTS In NON-HUMAN PRIMATES with Claudia Fichtel and Peter M. Kappeler

in Boos $M$, Kolbe M, Kappeler PM, Ellwart T (eds) Coordination in human and primate groups. Springer, Heidelberg, pp 37-56 (2011) 


\begin{abstract}
Many animals are organised into social groups. Because individuals have different preferences and diverging needs, conflicts of interests exist; these conflicts are particularly revealed and negotiated in the context of group movements. Thus, group movements provide an excellent example to study coordination processes in non-human primates. In this chapter we review several aspects related to group movements in nonhuman primates. We first summarise the current understanding of variation in spacing patterns, types of leadership, and decision-making processes. We then focus on methodological issues and discuss various operational definitions of group movements, and we propose an operational definition that has already been applied successfully in studies of small free-ranging groups. We conclude by discussing the possibilities and limitations of transferring concepts and methods from studies of non-human primate groups to research on human groups.
\end{abstract}

\title{
1 Introduction
}

Many animals are organised into permanent social groups. The shift from an originally solitary to a gregarious lifestyle is considered to be one of the major evolutionary transitions (Maynard Smith and Szathmáry 1995). These social groups differ enormously in size, composition, permanence, and cohesion (Parrish and Edelstein-Keshet 1999). Their members can be anonymous to each other, or they can recognise group or even individual identity. The ultimate reasons for why animals might be group-living as well as the respective optimal group size have been investigated in detail in diverse taxa (e.g., Bertram 1978; van Schaik 1983; Zemel and Lubin 1995). These evolutionary benefits include reduced individual predation risk, joint resource defence, cooperative foraging, shared vigilance, and information transfer (Alexander 1974; Bertram 1978).

Living in a group also leads to interindividual conflicts and costs, such as competition over resources and mates, as well as increased pathogen transmission. These factors limit the size of groups and act as a centrifugal force on group cohesion (Alexander 1974; Bertram 1978). First and foremost, individual foraging strategies and schedules are expected to be heterogeneous and are therefore a source of conflict. Growing juveniles, pregnant or lactating females, and adult males often have divergent overall activity budgets and different dietary needs, such as types of food items eaten and time devoted 
to foraging for each item (see, e.g., Altmann 1980; Dunbar and Dunbar 1988). Depending on the type and distribution of particular resources, intra-group feeding competition can threaten group cohesion and influence individual and subgroup movements (van Schaik 1989; van Nordwijk et al. 1993; Pulliam and Caraco 1984). A conflict of interest may also arise between the sexes when inter-group encounters have different costs and/or benefits for males vs. females (Cheney 1987) or when mating competition interferes with foraging efforts (Alberts et al. 1996).

In order to maintain group cohesion and social stability despite these conflicts, individuals need to synchronise and coordinate their activities such as foraging, resting, social interactions, and collective movements if they want to reap the benefits of gregariousness (Conradt and Roper 2003, 2007; Rands et al. 2003; Kerth et al. 2006). How this trade-off is achieved and implemented at the behavioural level is not easily studied. That said, natural group movements among resources provide an operationally accessible and ecologically relevant context to study these fundamental mechanisms of social coordination. In the context of group movements, it is possible to quantify how members of a group achieve a communal decision about which activities will be carried out, where, and for how long (Boinski and Garber 2000).

Because group movements are characterised by dynamics operating at multiple levels, it is heuristically useful to consider group movements on four different levels: (1) normative details of the spatio-temporal patterns of space use of a group as an entity such as travel routes within the home range and their variability according to seasonal changes and climatic conditions, resource availability, predation risk, and/or the likelihood of inter-group encounters; (2) behavioural processes describing who initiates, leads, and terminates a group movement and how many members follow whom; (3) communication mechanisms that control the processes proximately, such as vocal or visual signals used to initiate a movement and to maintain group cohesion; and (4) whether leadership is distributed or monopolised. If leadership is distributed, all group members are said to contribute to a democratic decision. If a single individual leads the group and the other group members merely follow, the decisions are said to be despotic (Conradt and Roper 2003, 2005). Because information on all four aspects is not available for most species (honey bees being an exception; see, for example, Seeley and Visscher 2004), general principles are currently best inferred from inter-specific comparisons. We 
adopt this approach and focus on one relatively well-studied taxon with interesting variation in social organisation: non-human primates. In this chapter we review the currently available information on group movements in non-human primates with special emphasis on the four levels described above. We then raise the issue of how group movements in animals can be operationalised by human observers in the field. Final thoughts provide a current context and future outlook on inter-disciplinary research in human and non-human primates.

\section{Group movements in non-human primates}

The more than 300 species of non-human primates are interesting subjects for the study of group movements for at least four reasons. First, they exhibit more variation in social organisation than most other vertebrate taxa. Primate groups range in size from two to several hundred individuals of both sexes and multiple generations (Smuts et al. 1986). Second, primates occupy a wide range of habitats, from semi-deserts to tropical rain forests and temperate mountain forests, resulting in movements that appear to be guided by these widely differing ecological needs (Eisenberg 1981). Third, non-human primates have larger brains relative to their body size than other mammals and vertebrates, suggesting that behavioural aspects of group movements may be influenced by their unusual cognitive abilities (Reader and Laland 2002; Dunbar and Shultz 2007). Finally, primates vary across species in dominance styles and predominant communication modalities (Seyfarth 1986; Zeller 1986; Sterck et al. 1997), offering interesting behavioural variation in the social component of group movements.

\subsection{Patterns of group movements}

Beside abiotic variables, ecological factors such as seasonal differences in resource distribution or predation risk, as well as social influences from neighbouring groups, affect daily ranging patterns of primate groups. We illustrate these effects with a few examples below.

The spatio-temporal distribution and availability of resources not only influence the size and cohesion but also the ranging patterns of primate groups (van Schaik 1983; Chapman et al. 1995). For instance, food availability has been observed to significantly affect activity profiles and habitat use of red-fronted lemurs (Eulemur rufifrons) and red- 
bellied lemurs (Eulemur rubriventer) in Ranomafana National Park, a rainforest in southeastern Madagascar (Overdorff 1993a, 1996). During periods of food scarcity, both species fed more and dedicated less time to travelling and resting. Red-fronted lemurs in Ranomafana also conducted group movements of up to $5 \mathrm{~km}$ away from their usual ranges during a period of fruit scarcity in order to exploit extraordinary food abundance (a guava plantation) elsewhere. The ranging behaviour of red-fronted lemurs was also affected by the differential availability of water during the dry and rainy seasons in the Kirindy Forest, a dry deciduous forest in western Madagascar. During the 8 month dry season, groups living close to ephemeral water holes made daily excursions of up to two to three home range diameters to drink, whereas groups living farther away from the river shifted their ranges nearer the water holes for several weeks or months and moved very little during this time (Scholz and Kappeler 2004). We also observed a group with permanent access to a water hole in their usual home range extending its range away from the riverbed (Pyritz et al., unpub. data). Presumably, lemurs exhibit this behaviour in order to avoid encounters with conspecifics from other groups or predators that are attracted by the lemurs gathering at the water holes in large numbers.

Resource availability has also been observed to influence travelling patterns in chacma baboons (Papio ursinus) (Noser and Byrne 2007a). During the dry season, the study group followed linear paths over great distances in the morning to reach sparse fruit trees and ephemeral waterholes. In the afternoons, when the baboons fed on seeds, group movements were shorter and sinuous. During the rainy season, food distribution determined the onset of group movements. The baboons left their sleeping sites earlier when visiting patchily distributed fig trees than when moving towards evenly distributed fruit resources. Therefore, these baboons seem to plan movements according to the type of feeding goal.

The presence of conspecific groups has also been observed to be an additional factor impacting the ranging behaviour of chacma baboons (Noser and Byrne 2007b). When neighbouring groups were present within a 500-m radius, the routes conducted by the focal group were less linear, the baboons travelled faster, and they covered larger distances between different resources. These changes in travelling behaviour are interpreted as measures to avoid group encounters, which can proceed quite aggressively in this species. 


\subsection{Processes and leadership}

The process of group movements depends on the species, group composition, and permanence. For example, fish swarms and bird flocks are often so large that members seem to neither know each other individually nor know which individuals possess decisive information, and they also appear to lack recruiting signals (Couzin et al. 2002). Cohesion and coordinated movements in such groups are often maintained by self-coordination such as individuals following the simple rule of 'keep a certain safe distance to the next neighbour (Parrish and Edelstein-Keshet 1999; Hemelrijk 2002; Couzin et al. 2002). In contrast, in groups where members know each other individually, such as primates, certain individuals may adopt different roles and initiate and terminate a group movement (Boinski and Garber 2000).

Studies of several primate species revealed that age, rank, or sex can be defining characteristics of group leaders. In many species, adult and therefore more experienced and knowledgeable individuals initiate and lead group movements more often than juveniles (Japanese monkeys, Macaca fuscata: Itani 1963; Costa Rican squirrel monkeys, Saimiri oerstedii: Boinski 1991; chimpanzees, Pan troglodytes: Boesch 1991a; white-faced capuchin monkeys, Cebus capucinus: Boinski and Campbell 1995; mountain gorillas, Gorilla gorilla: Stewart and Harcourt 1994). In some species, dominant animals rather than the most experienced lead groups more often than subordinate individuals (hamadryas baboons, Papio hamadryas: Kummer 1968; mountain gorillas: Watts 1994; white-faced capuchin monkeys: Boinski 1993; ringtailed lemurs, Lemur catta: Sauther and Sussman 1993). However, rank is often confounded with age or sex, which handicaps untangling the relative importance of these variables in structuring group leadership.

Many studies showed females to lead groups more often than males (see Table 1; Neville 1968; Rowell 1969; Struhsaker 1967a; Dunbar and Dunbar 1975; Oates 1977; van Nordwijk and van Schaik 1987; Boinski 1988; Mitchell et al. 1991; Erhart and Overdorff 1999; Leca et al. 2003; Trillmich et al. 2004). This sex difference is usually attributed to higher nutritional needs of females due to the energetic costs of gestation and lactation (Boinski 1988, 1991; Erhart and Overdorff 1999; Trillmich et al. 2004).

Reasons for male leadership are surmised to include dominance or mating competition (Table 1). For example, male mountain gorillas initiate group movements after contact with a rival (Watts 1994), and in spider monkeys (Ateles geoffroyi), males 
frequently lead their group to the edge of the home range presumably to make contact with females from other groups (Chapman 1990). Sex differences in leadership of groups have also been explained by sex-specific patterns of residency and dispersal and a corresponding improved information status of the philopatric sex regarding the distribution and availability of different resources (Struhsaker 1967b; Goodall 1968; Sigg and Stolba 1981; van Nordwijk and van Schaik 1987; Watts 1994; Trillmich et al. 2004).

Animals were identified as leaders when they had been observed initiating movement and were therefore at the forefront of collective movements. However, the initiating individual did not always remain in the leading position during the entire movement, meaning that either changes in their forefront positioning occurred (hamadryas baboons: Kummer 1968; guinea baboons, Papio papio: Byrne 1981, 2000) or the movement was terminated by an individual different from the initiator (indris, Indri indri: Pollock 1997). There are also reports of distributed leadership where all group members equally initiated and led movements (Leca et al. 2003; Meunier et al. 2006; Jacobs et al. 2008).

How and why leadership and followership evolved and how such a system can be stable have been the subject of a number of recent studies (e.g., Conradt and Roper 2005; Couzin et al. 2005; van Vugt 2006; Rands et al. 2008; Sueur and Petit 2008a). On the one hand, leadership is interpreted as a byproduct of dominance and submission in animal groups (e.g., Alexander 1987). Several other studies that mainly focused on non-primate species with no clear dominance hierarchy identified correlates of leaders, including intrinsic factors such as size or physiological state (Krause et al. 1998; Rands et al. 2003; Fischhoff et al. 2007), personality characteristics such as activity (Beauchamp 2000) and boldness (Ward et al. 2004; Leblond and Reebs 2006), positive social feedback between group members (Harcourt et al. 2009), and asymmetries in information or knowledge (Reebs 2000, 2001; Dyer et al. 2009 as an example for human groups). Because in most non-human primates, several individuals of a group may act as leaders, a combination of dominance, physiological state, personality characteristics, and also knowledge may explain why several individuals emerge as principal leaders of a group.

Although leadership also involves costs such as reduced attention (Piyapong et al. 2007), individuals that lead group movements have the advantage of promoting their own interests compared to followers. Hence, conflicts over the leading position would 
seem likely to arise (reviewed in Conradt and Roper 2005), but they are, in fact, rarely observed. Leading and following animals may simply differ in the degree of their incentives (Erhart and Overdorff 1998), or the long-term fitness benefits related to social ties or kinship could compensate for the short-term costs of following a leader in a given situation (Silk et al. 2003; Cheney and Seyfarth 2007; King et al. 2008; Sueur and Petit 2008a). Alternatively, following may simply not be costly in each and every case, so that these conflicts do not arise permanently.

\subsection{Mechanisms of group coordination}

Visual or acoustical displays are obvious signals to initiate group movements. Visual displays such as staring or intentional movements in the direction of the adopted course have been reported in several primate species (Table 1; reviewed by Boinski 2000). For example, the dominant male in mountain gorillas usually uses a simple characteristic gesture to initiate a movement: He walks stiff-leggedly and rapidly in a certain direction (Schaller 1963). Acoustical displays used to coordinate group movements, so-called travel calls (Boinski 1991), have also been reported for a number of primate species primarily from the New World (Boinski 1991, 1993; Boinski et al. 1994; Boinski and Campbell 1995; Boinski and Cropp 1999; Leca et al. 2003). In squirrel and capuchin monkeys, travelling is initiated when an individual (occasionally two or three) moves to the edge of the group and produces a specific travel call. Byrne (1981) observed the use of vocalisations in the context of group movements in Guinea baboons ( $P$. papio). The individuals exchange barks to stay cohesive as a group in areas of poor visibility (dense grass, thickets), as well as to coordinate themselves before the group splits up into subgroups or fusions.

Some species combine visual and acoustic displays. Barbary macaques (Macaca sylvanus) shake twigs or drum on dead wood (Mehlman 1996), and bonobos (Pan paniscus) have been observed dragging branches behind them to make their conspecifics move (Ingmanson 1996). However, in other species such as sifakas, the initiation of group movements is not accompanied by any acoustical or visual displays (Trillmich et al. 2004). The above description of inter-specific variation in the existence and type of initiation signals is extremely abbreviated. It is conceivable that future studies of additional species may reveal that the existence of initiation signals is a function of group size and cohesion, with species living in larger groups exhibiting specific calls to initiate travel, and that the 
existence of multiple signals is related to habitat characteristics that influence the propagation of certain signals.

\subsection{Decision types}

Group decisions can be defined as 'when the members of a group choose between two or more mutually exclusive actions with the aim of reaching a consensus' (Table 1; see also Conradt and Roper 2005). Decisions can principally be shared, unshared, or based on selforganised processes (Hemelrijk 2002; Conradt and Roper 2003, 2007). In all cases, decisions to perform a certain activity or to travel in a certain direction appear to ultimately be made by single individuals, but their consequences are manifested on the level of the group in the form of a communal decision. Only a few studies to date have described decision-making processes in non-human primates. In capuchin monkeys and Tonkean macaques (Macaca tonkeana), each individual can principally influence the travel direction, resulting in a shared-consensus decision-making process; whereas in Rhesus macaques, dominant and older group members take a prominent role, resulting in only partially shared consensus decisions (Leca et al. 2003; Meunier et al. 2006; Sueur and Petit 2008a, b).

A despotic decision-making process has been described in mountain gorillas. In this species, the entire daily routine - the time of rising, the distance and direction of travel, as well as the place and time of nest building - is determined by the silverback male. When he starts moving in a certain direction, the whole group, which seems to be constantly aware of the location and activity of the dominant male, follows (Schaller 1963). Conflicting results have been reported regarding decision-making processes in baboons. In one population, King et al. (2008) conducted a foraging experiment with two wild chacma baboon groups and found that the dominant male of the group consistently led all foraging movements to experimental feeding sites. Social ties are held responsible for subordinate individuals following the despotic leader. In contrast, Stueckle and Zinner (2008) observed in another population of chacma baboons a democratic decision-making process during their departure from the sleeping site, with adult males contributing more to the decision outcome than adult females. Thus, differences in decision making processes either might be related to taxonomic differences or may vary according to the decision that has to be made: It was observed that going to a feeding site that can be 
monopolised by the dominant male resulted in a despotic decision, whereas the departure from the sleeping site at dawn, which probably all group members want to leave to move on to forage, resulted in a democratic decision. Divergent or common context-dependent interests of group members may therefore result in different decision processes.

\section{Operationalisation of group movements in the field}

Because coordination processes are mostly studied in the context of group movements, we would like to raise the issue of how human observers can identify and operationally define such a movement. In fact, group movements do not always proceed in a coordinated manner and, therefore, cannot always be easily captured by a single definition. For example, several or all animals of a group sometimes travel during foraging activities ('feed-as-you-go'), resulting in amoeboid-like movements that do not necessarily require an initiator or coordination among group members (e.g., bonobos, Pan paniscus: Wrangham 2000). Therefore, it is important to separate those movements from directed movements between sleeping and feeding sites or movements to patrol the border of the home range which require a certain degree of coordination among group members (Boinski and Garber 2000; Kappeler 2000; Pyritz et al. 2010).

Early studies addressing questions about leadership, coordination processes, and communication mechanisms in collective movements employed rather basic and unspecific definitions. One of the first definitions was provided by Altmann (1979), who defined a group movement simply as 'a displacement of the centre of the group.' Because a displacement of the centre also occurs during the rather amoeboid-like foraging movements, this definition does not allow differentiating between the latter and more coordinated movements. In other studies, group movements were defined by the departure of the group from a resting or feeding site (Schaller 1963; Stewart and Harcourt 1994; King et al. 2008), but this definition may not capture all movements. According to definitions of more recent studies that specifically address questions about the coordination of group movements, a group movement starts when an individual moves a certain distance towards the edge of the troop in a defined time period, e.g., $10 \mathrm{~m}$ within 40 s (Leca et al. 2003; Sueur and Petit 2008a, b; Stueckle and Zinner 2008), and is followed by at least one conspecific. Although these definitions have the advantages of 
being more precise, less presumptuous regarding resting and foraging motives, and intersubjectively comprehensible, the distance that had to be travelled in a certain timeframe to initiate a group movement was not established empirically with regard to the speciesspecific travel pattern. Boinski $(1991,1993,2000)$ defined group movements in a number of New World monkeys by a specific travel call uttered by the initiating individual, but because not all species produce specific travel calls, this definition is not generally applicable. Some researchers therefore use combinations of the definitions described above (e.g., Erhart and Overdorff 1999).

In general, a definition of group movements has to include a number of different travelling types: Primates do not only move between feeding and resting sites, but also to patrol home range boundaries and/or to search for or to avoid neighbouring groups. Because groups of different primate taxa vary widely in size, composition, and cohesion, which has consequences for home range size and travel distances, the minimum meaningful distance an individual has to cover to initiate a movement as well as the definition of the corresponding followers behaviour have to be species specific (Pyritz et al. 2010). Below we suggest a procedure to generate an operational definition of group movements for different taxa built upon empirical data collected during a pilot study (Pyritz et al. 2010).

In order to define objective rules for directed vs. amoeboid-like movements, we suggest observing a number of randomly chosen focal animals for a period of several days. During such a pilot study, any movement of more than a body length can be recorded and estimated to the nearest metre. In addition, the latency between two movements, the total distance covered, as well as the distance to the nearest neighbours after the end of the movement can be noted. Based on meaningful breaks in the corresponding frequency distributions, a movement can be defined as follows:

Start: An individual has been stationary for at least $\mathrm{x}$ minutes and then moves a minimum of $x$ metres in a directed manner without pausing.

Initiator: The individual that started the movement is the initiator.

Leadership: The individual at the forefront of the moving group is considered to lead the group movement.

Takeover: An individual overtakes the leader by more than several body lengths without diverging more than $45^{\circ}$ from the initial trajectory of travel. 
Followers: Group members moving behind the leader are termed followers unless their movements diverge more than $45^{\circ}$ from the leader's trajectory. If they differ by more, the individual's movement is regarded as a separate movement. Followers have to arrive within an $\mathrm{x}$-metre radius around the terminator, no later than $\mathrm{x}$ minutes after termination of the movement.

Termination: The end of the movement occurs when the leader is stationary again for at least $\mathrm{x}$ minutes (see above definition for 'Start').

Regarding these definitions, it is important to keep in mind that the initiator does not always remain the leader during the entire movement (hamadryas baboons: Kummer 1968; Guinea baboons: Byrne 1981, 2000) and that the terminator can differ from the initiating individual (indris: Pollock 1997). In the Kirindy Forest, we recorded group movements of red-fronted lemurs according to the above definition with two observers: one following the initiator, the other following the leader in case a change of leadership occurred (Pyritz et al., unpub. data). A number of times the overtaking animal was only followed by a portion of group mates. This subgroup later returned to the other individuals, which were grouped around the original initiator, who had continued leading the rest of the group. Hence, the initiator still functioned as the pace-maker of the movement, even after being temporarily overtaken by a new leader. Hidden leadership such as this has to be taken into account when defining the decision type of a certain species. Furthermore, it highlights the importance of at least two observers following groups on the move.

We also studied group movements in Verreaux's sifakas using the method introduced above. The two species are syntopic but differ in both group and home range size. The virtually exclusively arboreal Verreaux's sifakas in the Kirindy live in multi-male, multi-female groups, with an average of 4.1 adult individuals per group that occupy home ranges averaging 7.3 ha (Benadi et al. 2008; Kappeler and Schäffler 2008). The cathemeral red-fronted lemurs also live in multi-male, multi-female groups composed of on average 5.6 adult individuals (Kappeler and Port 2008). Average home range size of this species is 18 ha in the Kirindy (Pyritz et al., unpub. data). Red-fronted lemurs spend a significant proportion of their time on the ground, especially during long group movements.

In Verreaux's sifakas, we employed the following group movement definition: A start attempt is made when an individual is stationary for at least $4 \mathrm{~min}$, then moves at 
least $5 \mathrm{~m}$, and is followed by at least one group mate. Other group members were termed followers unless their movement diverged more than $45^{\circ}$ from the trajectory of the movement of the initiator. A movement was considered terminated when the leading individual was again stationary for at least 4 min (Trillmich et al. 2004). By applying this definition, we found that both sexes initiated group movements but that females did so more often, led groups over greater distances, and enlisted more followers than males. Presumably, this more active role enables females to positively influence their individual foraging efficiency and nutritional intake, especially during gestation and lactation (see Boinski 1991; Erhart and Overdorff 1999). However, the sex of the leader had no effect on the probability that a group would feed or rest after a successful movement. A certain vocalisation, the so-called grumble, was emitted by both leaders and followers at high rates, both before and during group progressions, but grumbles uttered just before an individual moved were characterised by a significantly steeper frequency modulation at the beginning of the call and higher call frequencies in both females and males (Trillmich et al. 2004). The results of this study indicate that sifakas converge with many other group-living primates in several fundamental proximate aspects of group coordination and cohesion. In contrast to many other primates, however, sifakas do not use a particular call or other signals to initiate or control group movements.

Our earlier pilot study suggested a group movement definition similar to the one employed for sifakas for the ongoing study on coordination of group movements in redfronted lemurs: A movement is initiated when an individual is stationary for at least 4 min, then moves at least $15 \mathrm{~m}$, and is followed by at least one group mate. A movement was considered terminated when the leading individual was again stationary for at least 4 min. Followers are defined as individuals moving behind the initiator without diverging more than $45^{\circ}$ from the trajectory, arriving within 6 metres proximity to the terminator, and no later than $10 \mathrm{~min}$ after termination. Preliminary results suggest that adults of both sexes initiated movements but that females do so significantly more often, both during the day and at night. Socially powerful males, so-called central males (Ostner and Kappeler 1999), did not initiate or lead group movements more often than other males. Female prevalence concerning the initiation of group movements may be due to higher and more complex nutritional needs during times of reproduction or female philopatry, 
but this should be true for most primates and other mammals. No specific initiation movements or travel calls have been observed thus far.

Comparing the operational definitions used in these two studies revealed that only the criteria for the start of a group movement and for the followers varied between sifakas and red-fronted lemurs: The distance travelled for initiation of a group movement varied between $5 \mathrm{~m}$ in sifakas to $15 \mathrm{~m}$ in red-fronted lemurs, and time intervals used to determine followers at the end of a group movement varied between $4 \mathrm{~min}$ in sifakas to $10 \mathrm{~min}$ in red-fronted lemurs. Both sets of criteria clearly reflect the difference in daily path length as well as home range size [daily path length for sifakas: $1.1 \mathrm{~km}$, home range size: 4.5 ha (Trillmich et al. 2004); daily path length for red-fronted lemurs: $2 \mathrm{~km}$, home range size: 18 ha (Pyritz et al., unpub. data)] and group size between species in the Kirindy, indicating that the use of such an operational group definition indeed helps to develop an appropriate way to quantify species-specific group movements. We therefore hope that future studies of primate group movements will continue to use, and eventually converge upon, similar criteria, increasing the potential for meaningful interspecific comparisons.

\section{Interdisciplinary outlook}

Although group cohesion and group decision making, both among humans as well as in non-human primates, are interesting in their own right, evolutionary theory suggests that both have to be functional with regard to environmental factors. In this respect, primatology and anthropology, on the one hand, and social psychology, on the other hand, differ considerably in their approaches. Primatology and anthropology focus on the long-term success of group cohesion and group decision making; that is, they ask what patterns are functional for group stability and the survival of group members. In contrast, psychological research focuses more on the short-term success of group cohesion and the mechanisms and processes underlying group decision making. For example, social psychologists are interested in whether group processes in terms of information exchange or mutual understanding benefit from cohesion or specific types of cohesion (Cornelius and Boos 2003), or how highquality decisions can be achieved in groups (Schulz-Hardt et al. 2006). 
Hence, comparative research on the consequences of group cohesion, group decision making, and other group processes on performance criteria in human vs. nonhuman primate groups could offer new insights for both disciplines. For instance, the short-term consequences of group processes on performance could be investigated in non-human primate groups. For example, it remains unknown to what extent the same process losses and gains that have been found in human groups also exist among nonhuman primates. Such an investigation of group specific influences on non-human primates' task-related performance would be interesting in itself (e.g., studying capability gains among non-human primates as a function of social learning in a group), and might also significantly contribute to our understanding of human group performance. An open question in research on motivation gains in groups is why group members exert extra effort in a group situation under specific conditions. Whereas some approaches trace this behaviour back to a selfish motive (e.g., winning the performance competition and thereby gaining status in the group), other approaches postulate a more prosocial motive (e.g., caring for the group's welfare).

Since most primate species most likely lack collectivistic motivations or prosocial tendencies, whereas individualistic motives such as striving for status can be frequently found among them, comparative studies of group vs. individual performance in tasks such as predator mobbing or inter-group encounters where performance almost exclusively depends on effort could provide interesting new evidence for this open question. It also seems feasible that studies of human groups could take advantage of the long-term perspective adopted in non-human primate group research. By more extensively studying real groups in the field over extended periods of time, a more adequate picture of 'successful' human group behaviour might arise. Specifically, we might learn to what extent processes that directly impede the short-term performance of groups might nevertheless be facilitative or even essential for the stability and survival of a group in the long run. 


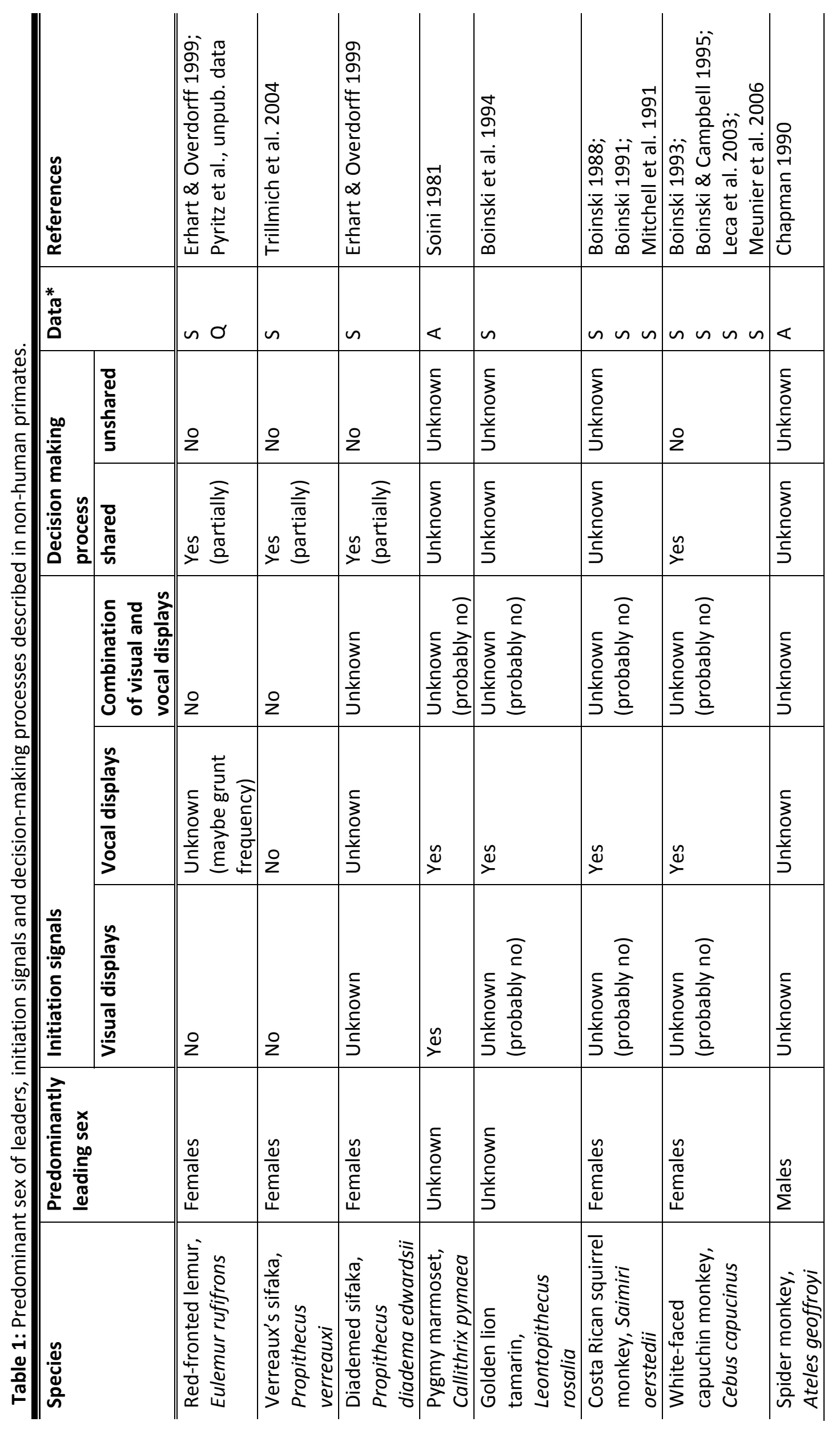




\begin{tabular}{|c|c|c|c|c|c|c|c|c|c|c|}
\hline \multicolumn{2}{|l|}{ 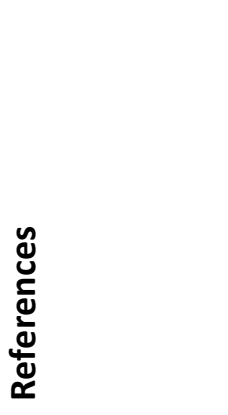 } & 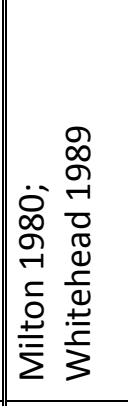 & 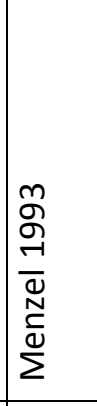 & 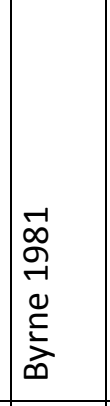 & 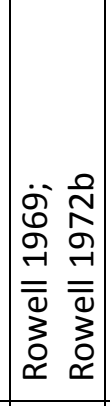 & 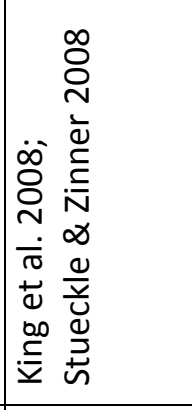 & 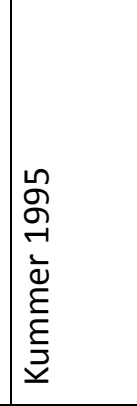 & 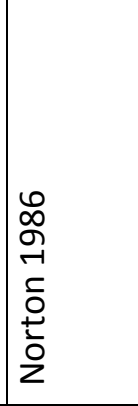 & 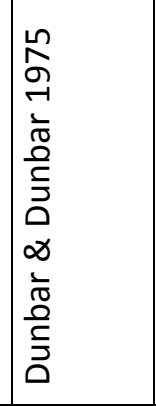 & 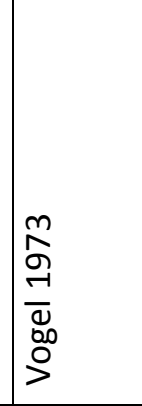 \\
\hline \multicolumn{2}{|l|}{ 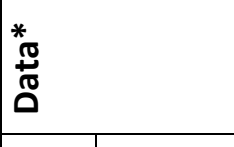 } & $\ll<$ & $\backsim$ & us & $\ll<$ & $n n$ & $n$ & $\varangle$ & $\ll$ & $\ll$ \\
\hline \multirow{2}{*}{ 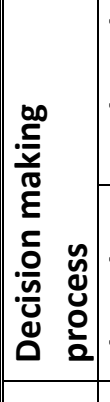 } & $\begin{array}{l}\frac{8}{d} \\
\frac{d}{\pi} \\
\frac{5}{n} \\
\frac{5}{5}\end{array}$ & \begin{tabular}{|l}
$\frac{5}{3}$ \\
0 \\
$\frac{5}{5}$ \\
5 \\
5
\end{tabular} & $\begin{array}{l}\frac{5}{3} \\
0 \\
\frac{5}{5} \\
\frac{5}{5} \\
\end{array}$ & $\begin{array}{l}\frac{5}{3} \\
0 \\
\frac{5}{5} \\
5 \\
5\end{array}$ & $\begin{array}{l}\frac{5}{3} \\
0 \\
\frac{5}{5} \\
5 \\
5\end{array}$ & 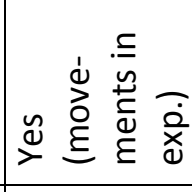 & $\frac{1}{2}$ & $\begin{array}{l}\frac{5}{3} \\
0 \\
\frac{5}{5} \\
\frac{5}{5}\end{array}$ & $\begin{array}{l}\frac{c}{3} \\
0 \\
\frac{5}{5} \\
\frac{5}{5} \\
\end{array}$ & 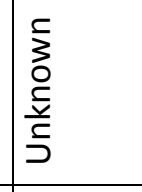 \\
\hline & & \begin{tabular}{|l} 
\\
$\frac{5}{2}$ \\
$\frac{5}{5}$ \\
$\frac{5}{5}$ \\
\end{tabular} & \begin{tabular}{|l}
$\frac{5}{3}$ \\
0 \\
$\frac{5}{5}$ \\
$\frac{5}{5}$ \\
\end{tabular} & \begin{tabular}{|l} 
\\
$\vdots$ \\
0 \\
$\frac{5}{5}$ \\
5 \\
5 \\
\end{tabular} & 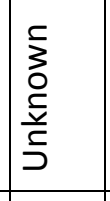 & 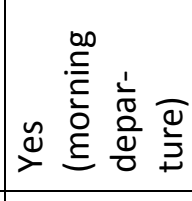 & 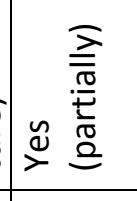 & \begin{tabular}{|l}
$\frac{5}{3}$ \\
0 \\
$\frac{5}{5}$ \\
$\frac{5}{5}$ \\
\end{tabular} & \begin{tabular}{|l}
$\frac{5}{3}$ \\
0 \\
$\frac{c}{5}$ \\
$\frac{5}{5}$ \\
\end{tabular} & \begin{tabular}{|l}
$\frac{5}{3}$ \\
0 \\
$\frac{c}{5}$ \\
$\frac{5}{5}$ \\
\end{tabular} \\
\hline \multirow[b]{3}{*}{ 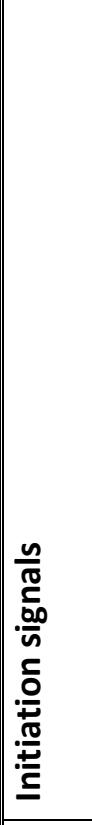 } & 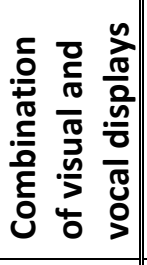 & 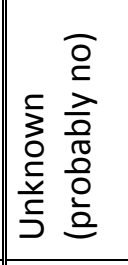 & 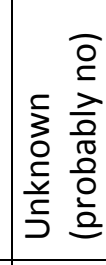 & 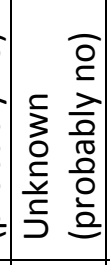 & \begin{tabular}{|l|l|} 
& 0 \\
5 & 0 \\
3 & 0 \\
0 & 0 \\
0 \\
$\frac{5}{5}$ & 0 \\
5 & 0 \\
5 & 0
\end{tabular} & \begin{tabular}{|l} 
\\
$\frac{1}{3}$ \\
0 \\
$\frac{1}{5}$ \\
$\frac{5}{5}$ \\
\end{tabular} & 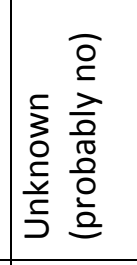 & 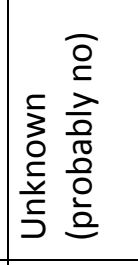 & 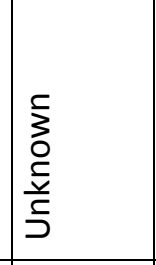 & 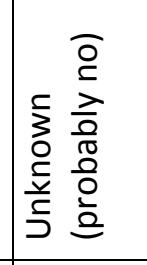 \\
\hline & 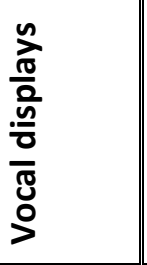 & 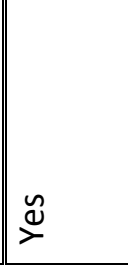 & 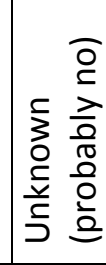 & $=\stackrel{\infty}{\infty}$ & 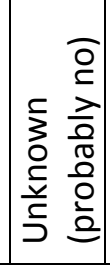 & 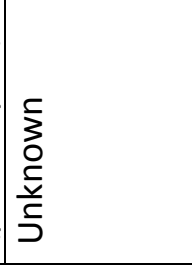 & 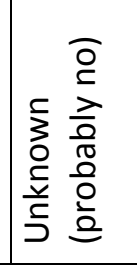 & 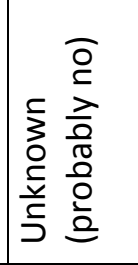 & 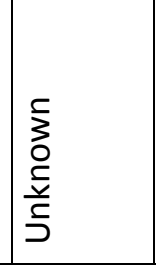 & 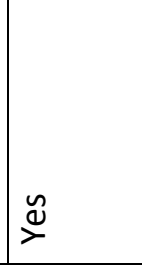 \\
\hline & 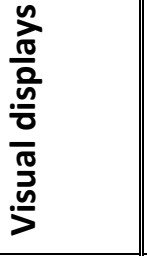 & 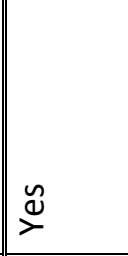 & $\stackrel{\tilde{\Delta}}{\rightleftharpoons}$ & 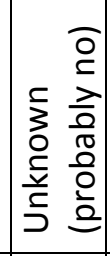 & $\stackrel{\infty}{-}$ & \begin{tabular}{|l}
$\frac{F}{3}$ \\
0 \\
$\frac{5}{5}$ \\
J \\
\end{tabular} & $\stackrel{\varrho}{\rightleftharpoons}$ & $\stackrel{\Perp}{\rightleftharpoons}$ & $\begin{array}{l}\frac{5}{3} \\
\frac{0}{5} \\
\frac{5}{5} \\
\end{array}$ & 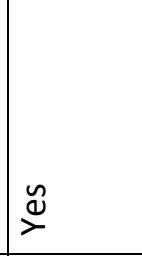 \\
\hline \multicolumn{2}{|c|}{ 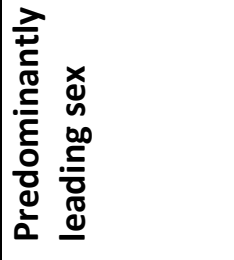 } & 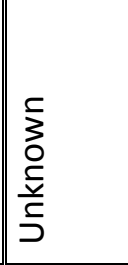 & $\begin{array}{l}\frac{5}{3} \\
\text { o } \\
\frac{5}{5} \\
د\end{array}$ & 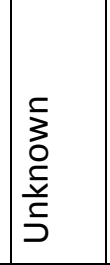 & \begin{tabular}{|l}
$\frac{\tilde{\omega}}{\tilde{\sigma}}$ \\
$\tilde{\Xi}$ \\
$\Psi$ \\
\end{tabular} & \begin{tabular}{|l}
$\frac{5}{3}$ \\
0 \\
$\frac{5}{5}$ \\
J
\end{tabular} & 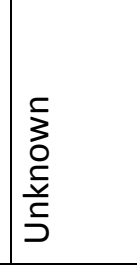 & 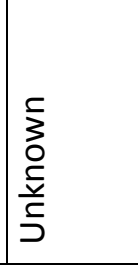 & 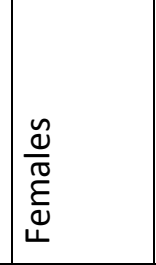 & 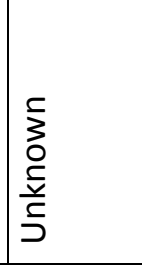 \\
\hline \multicolumn{2}{|l|}{ 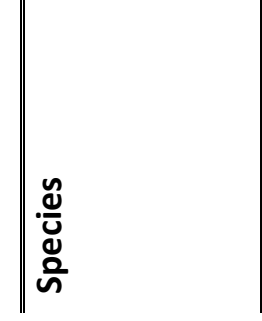 } & 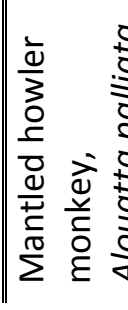 & 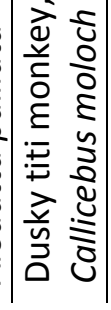 & 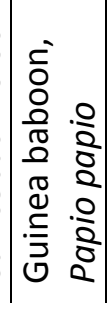 & 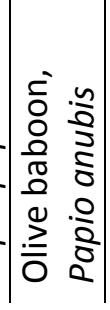 & 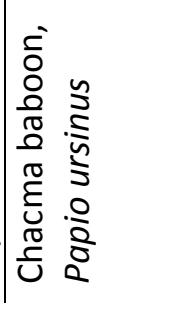 & 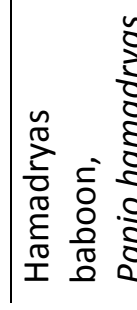 & 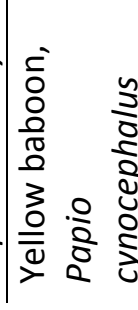 & 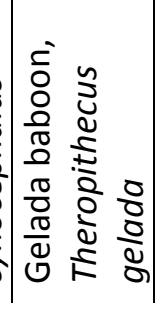 & 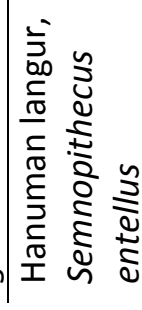 \\
\hline
\end{tabular}




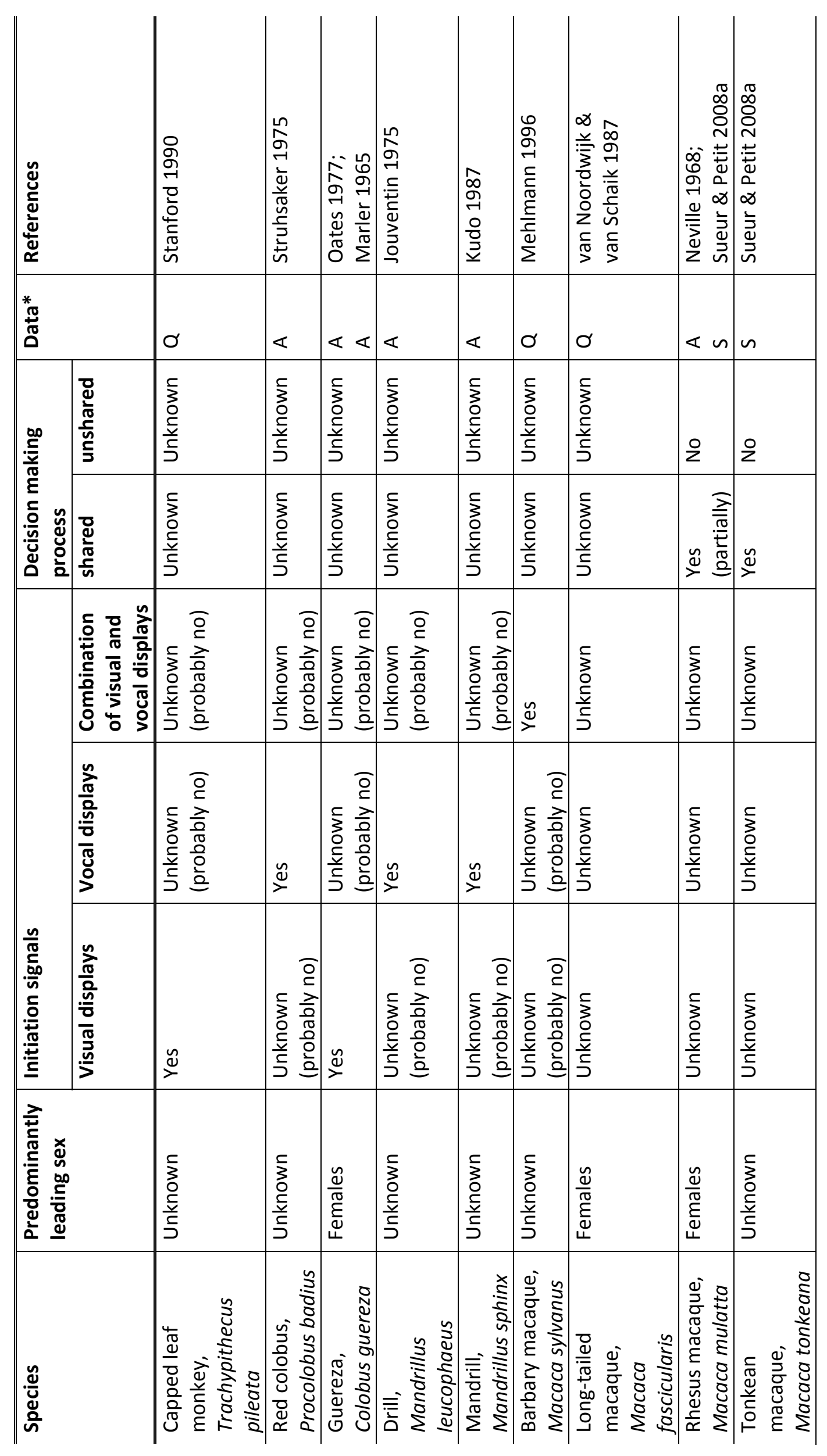




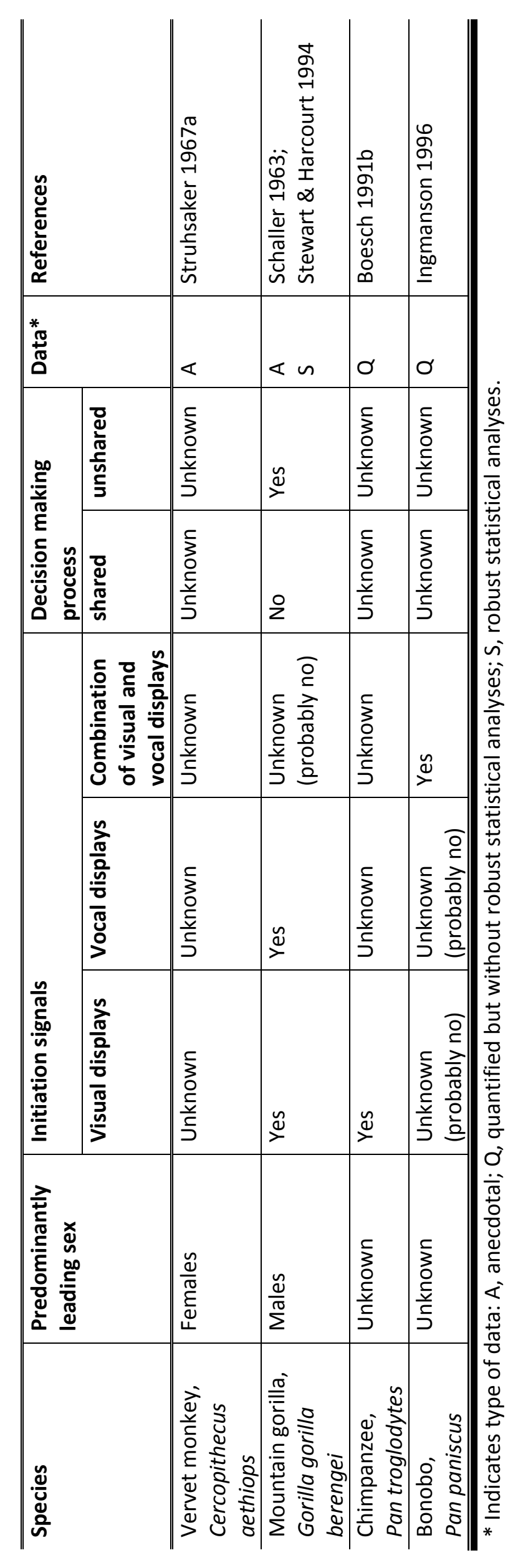


CHAPTER 1.2

Conceptual and Methodological IsSUes in the Comparative Study of Collective Group Movements

with Claudia Fichtel and Peter M. Kappeler

Behavioural Processes 84: 681-684 (2010) 


\begin{abstract}
In our commentary, we highlight several conceptual and methodological problems that have hampered broader integration of studies of collective group movements. Specifically, we argue that studies of captive animals should only be used to elucidate behavioural mechanisms. Moreover, the diversity of physical environments in which group movements occur as well as the social diversity of groups deserve more consideration in integrative studies. Furthermore, tests of predictions based on modelling studies are often hampered by the fact that models include variables that are difficult or impossible to measure in real animals. We also advocate the use of an empirical, rather than subjective establishment of operational definitions of group movements and the associated individual roles. Finally, we emphasize the utility of controlled experiments in the study of collective decision-making and group movements and encourage their wider application.
\end{abstract}

\title{
1 Introduction
}

Mobility is the key defining feature of animals that distinguishes them from plants. In many animal species two or (many) more individuals swim, walk or fly together in a coordinated fashion. Collective animal movements have been identified as an interesting topic in animal behaviour, cognition and ecology about a decade ago (Boinski and Garber 2000), and they continue to attract a lot of theoretical and empirical research (summarized in Petit and Bon, this volume; see also Conradt and List 2009; Kerth 2010). Despite the existence of several theoretical models and empirical studies of a number of species, the study of collective group movements is still in a phase of establishing consensus on a common nomenclature and methodology. The main aim of this commentary is, therefore, to highlight some of the current methodological and conceptual problems and challenges of studying group movements, and to offer some constructive suggestions.

\section{Conceptual obstacles to integration}

The target paper by Petit and Bon (2010) emphasizes similarities in the diversity of observed group movements in different species. These authors conclude and postulate that "the same scenario probably underpins nearly all cases of collective movements, but 
the balance between individualized and self-organized mechanisms to the global decision-making process will differ according to the environmental context, group size, the degree of sociality of the considered species and its cognitive capacities". We underscore the second part of this conclusion, emphasizing the fact that species- and context-specific variables will continue to introduce unexplained variability and idiosyncrasies that continue to hamper successful integration.

Here, we want to highlight two such points that we feel have not received sufficient consideration in existing reviews. First, the combination of results from studies in the wild and in captivity into a single analytical framework is problematic at some levels. Whereas captive studies can offer an advantage in terms of studying details of behavioural mechanisms underlying decision-making or the subsequent group movements, they clearly lack ecological relevance, so that studies of initiation and leadership, for example, can only generate preliminary insights, at best. We propose that group movements in an area of a few percent of a natural home range, in the absence of predators and with ad libitum food have no external validity. They should therefore be treated with caution or excluded from future comparative studies of group movements that do not deal directly with mechanisms of communication.

Second, fundamental species-specific constraints acting upon mobility and group cohesion deserve more attention. Aquatic and volant animals move in a threedimensional world. So do arboreal terrestrial species. Strictly terrestrial species or those requiring specific surfaces for locomotion (e.g., arboreal ants) move in a two-dimensional environment, however. This difference not only influences the animals' abilities to achieve and maintain cohesion, but also our abilities to operationalize and to quantify group movements. Moreover, in some environments or circumstances not moving is not an option. For example, the ecologically most spectacular examples of group movements involving pelagic crustaceans or fish, and migratory locusts or birds are predominantly characterized by their continuity, so that some questions typically asked about group movements are simply irrelevant. Finally, group size and stability, the degree of individual recognition and the dominant mode of communication are a set of similarly important species specific features that can act as constraints on some aspect of group movements. To us, these appear to be the minimum number of variables that need to be controlled for in future comparative studies attempting integration across species. 


\section{Theory meets biology}

As in the evolution of most other topics in behavioural biology, an initial descriptive phenomenological phase in the study of group movements has now given way to a hypodeductive stage. Models deduced from initial observations have not only formalized regularities, but also generated additional, new predictions that await testing. At this stage, it is particularly important to intensify the dialogue between theoreticians and empiricists and to scrutinize the biological relevance of the variables used in modelling approaches, in particular.

Although the number of empirical studies has been growing steadily, there is still a gap between some of the assumptions derived from theoretical models and the data available on coordination mechanisms in free-ranging animals, especially in vertebrates. Therefore, more field studies on non-anonymous groups are urgently needed to provide a broader basis for future modelling. Furthermore, most of the current theoretical models address only single correlates of leadership, i.e. energetic reserves (Rands et al. 2003), or assume simple behavioural rules that govern coordination (i.e. Couzin et al. 2005), while neglecting more sophisticated cognitive abilities. However, empirical studies revealed that coordination in animal groups can be highly complex and that it depends on several variables (Franks et al. 2003; Biro et al. 2006; Kerth et al. 2006; King et al. 2008). Therefore, long-term and comparative empirical studies are needed to identify reliably the mechanisms underlying leadership and decision-making in different contexts.

Another problem relates to operationalization, i.e. the principal measurability of theoretical variables in practical situations. Parameters like individual states of information or knowledge, individual degrees of assertiveness, energy level or susceptibility to predation (Rands et al. 2003, 2008; Couzin et al. 2005; Conradt et al. 2009) cannot be tested at all or can only be assessed very indirectly or crudely in the field. Therefore, theoretical models should be based on variables that can be observed and quantified empirically, such as nutritional intake, for example. On the other hand, practical studies have to incorporate more experimental approaches that allow for targeted analyses of single variables in an otherwise controlled environment or should compare a species' behaviour in different contexts. At the next higher level of comparison, a consensual methodological framework is desirable, to the extent that is can be meaningfully applied to species sharing similar environments and social features 
(see above). In their target paper, Petit and Bon (2010) have proposed a definition of a collective group movement. We complement this proposal with a methodological framework for operational approaches, using small groups of non-anonymous primates moving both on the ground and in a three-dimensional forest habitat as our example.

\section{Operational group movement definitions}

How human observers can reliably identify and operationally define a collective group movement in the field remains a major practical difficulty in many studies. In fact, group movements do not always proceed in a coordinated manner, but are sometimes also amoeboid, and can, therefore, not always be easily captured by a single definition (for details see Fichtel et al. 2011). According to definitions of recent studies which specifically addressed questions about the coordination of group movements, a collective movement starts when an individual moves a certain distance towards the edge of the troop (1) in a defined time period (e.g., 10 m within 40 s) (e.g., Leca et al. 2003; Sueur and Petit 2008a, b; Stueckle and Zinner 2008) or (2) without stopping and with the head raised (Bourjade et al., 2009; Ramseyer et al., 2009a, b, c) and is followed by a certain number of conspecifics. Although these definitions are quite precise and inter-subjectively comprehensible, the distance that had to be travelled in a certain time frame to initiate a group movement and criteria for a successful recruitment process were not established empirically with regard to the species-specific movement pattern. Boinski defined group movements in a number of New World monkeys by the occurrence of a specific travel call uttered by the initiating individual (Boinski 1991, 1993), but this definition is also not universally applicable because not all species produce specific travel calls (Fichtel et al. 2011; Petit and Bon 2010).

In general, a definition of group movements has to account for a number of different travelling types, i.e. animals move between feeding and resting sites, but also patrol home range boundaries or try to avoid neighboring groups. Additionally, different taxa vary widely in the environment in which they move (see above), as well as the size, composition, and cohesion of the group, which also effects home range size and travel distances. Therefore, the minimum meaningful distance an individual has to cover to initiate a movement as well as the definition of the corresponding followers' behaviour have to be species-specific. Below we suggest a procedure to generate an operational 
definition of group movements for different taxa built upon empirical data collected during a pilot study.

In order to approach an objective differentiation between directed and amoeboid movements, we suggest observing a number of randomly chosen focal animals for a period of at least several days. This requires that animals can be distinguished individually. During such a pilot study, any movement of more than a body length can be recorded and estimated to the nearest meter or other relevant unit. In addition, the latency between two movements (Fig. 1a), the total distance covered (Fig. 1b), as well as the distance to the nearest neighbour after the end of a movement (Fig. 1c) can be noted. Based on meaningful breaks in the corresponding frequency distributions (see the arrows in Fig. 1a-c) a number of parameters for a group movement can be estimated as follows:

Start: An individual has been stationary for at least $\mathrm{x}$ minutes and then moves a minimum of $x$ metres in a directed manner without pausing.

Initiator: The individual that started the movement is the initiator. The initiation may be difficult to observe in large or low-cohesion groups or in dense habitats, however.

Leadership: The individual at the forefront of the moving group is considered to lead the group movement.

Takeover: An individual overtakes the leader by more than several body lengths without diverging more than $45^{\circ}$ from the initial trajectory of travel.

Followers: Group members moving behind the leader are called followers unless their movements diverge more than $45^{\circ}$ from the leader's trajectory. If they differ by more, the individual's movement is regarded as a separate movement. Followers have to arrive within a $\mathrm{x}$ meter-radius around the terminator, no later than $\mathrm{x}$ minutes after the termination of the movement.

Termination: The end of the movement occurs when the leader is stationary again for at least $x$ minutes (see Start).

Hidden leadership: The individual that initiates a movement also terminates it, independent of whether it moves in the front of the group.

Regarding these definitions, it is important to keep in mind that the initiator does not always remain at the head of the group during the entire movement and that the terminator can differ from the initiating individual. We employed the above definition in a study of group movements of red-fronted lemurs with two observers: one following the 
initiator, the other following the leader in case a change of leadership occurred. A number of times the overtaking animal was only followed by a subgroup but not all group members. This proportion of group mates later returned to the other individuals that were grouped around the original initiator who had continued leading the rest of the group. Hence, the initiator still functioned as the pace-maker of the movement, even after being overtaken by a new leader. Hidden leadership such as this has to be taken into account when defining the decision type of a certain species. Furthermore, it highlights the importance of at least two observers following groups on the move.

We also studied group movements in Verreaux's sifakas (Propithecus verreauxi) using the method introduced above (for details see Trillmich et al. 2004). The two species are syntopic but differ in both group and home range size. The virtually exclusively arboreal Verreaux's sifakas live in multi-male, multi-female groups with an average of 4.1 adult individuals per group that occupy home ranges averaging 7.3 ha (Benadi et al. 2008; Kappeler and Schäffler 2008). The cathemeral red-fronted lemurs also live in multi-male, multi-female groups composed of on average 5.6 adult individuals (Kappeler and Port 2008). Average home range size of this species is 18 ha (Pyritz et al., unpub. data). Only red-fronted lemurs spend a significant proportion of their time on the ground, especially during long distance group movements.

Comparing the operational definitions used in these two studies revealed that only criteria for the start of a group movement and for the followers varied between sifakas and red-fronted lemurs: distance travelled for the initiation of a group movement varied between $5 \mathrm{~min}$ sifakas to $15 \mathrm{~min}$ in red-fronted lemurs, and time intervals used to determine followers at the end of a group movement varied between $4 \mathrm{~min}$ in sifakas to $10 \mathrm{~min}$ in red-fronted lemurs. Both sets of criteria clearly reflect the species difference in daily path length as well as home range size (daily path length for sifakas: $1.1 \mathrm{~km}$, home range size: 4.5 ha (Trillmich et al. 2004); daily path length for red-fronted lemurs: $2 \mathrm{~km}$, home range size: 18 ha (Pyritz et al., unpub. data) and group size, indicating that the use of such an operational group definition indeed helps to develop an appropriate way to quantify species-specific group movements. We therefore hope that future studies of animal group movements will continue to use, and eventually converge upon, similar criteria, increasing the potential for meaningful inter-specific comparisons. 

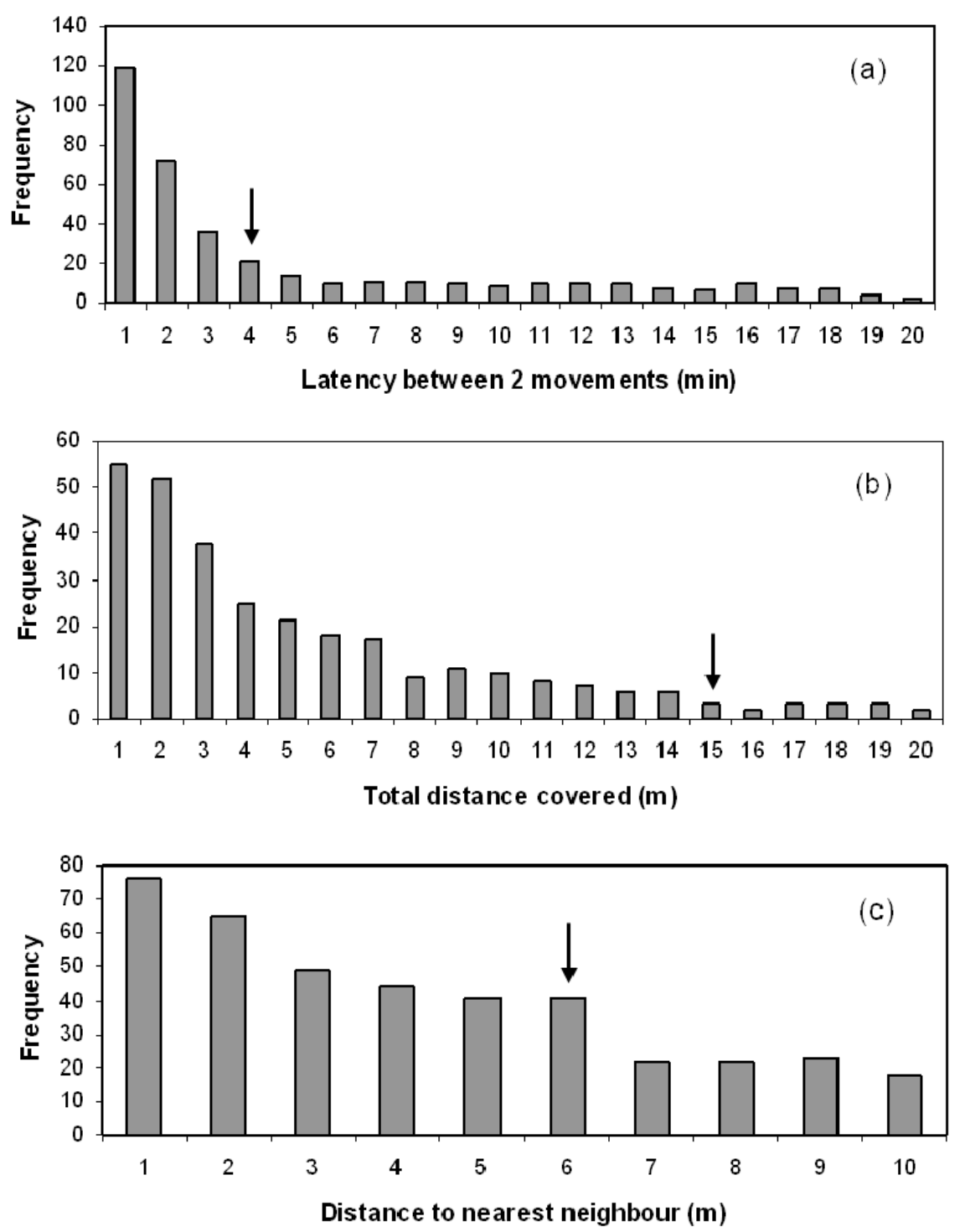

Fig. 1: Frequency distributions of (a) latencies between two movements $(n=381),(b)$ the total distance covered $(n=299)$ and (c) the distance to the nearest neighbor after the end of the movement ( $n=401$ ) collected during a pilot study on red-fronted lemurs (Eulemur rufifrons) at the field station of the German Primate Center in the Kirindy Forest, Western Madagascar by Pyritz in 2007. The arrows indicate the estimated thresholds for the operational group movement definition derived from the frequency distributions (a: 4min, b: $15 \mathrm{~m}, \mathrm{c}: 6 \mathrm{~m}$ ).

\section{Field experiments: The next frontier}

Group decisions can be provoked and analyzed by manipulating the level of interindividual conflict among group members (e.g., Biro et al. 2006; Kerth et al. 2006; King et al. 2008). In most species, however, it will be difficult or impossible to change group composition or to artificially augment encounters with other groups or predators to systematically test the effects of conflicts on decision-making and group movements. But 
varying information regarding resources provides a promising experimental tool in field studies, given that intrinsic differences regarding the energy balance among group members are sources of conflict and probable incentives for leadership and decisionmaking (Rands et al. 2003; King et al. 2009).

Several studies have already moved on to study collective decision-making and group movements experimentally. Meunier et al. (2006) videotaped groups of capuchin monkeys (Cebus capucinus) trained to the sound of a whistle as they had to decide between two different mangers, one of them filled with figs, located in opposite corners of their enclosure. Individual foraging benefits could not be assessed in this study, though, because each manger allowed all monkeys of a group to feed simultaneously and individual food intake was not quantified. King et al. (2008) provided two groups of wild chacma baboons with experimental food patches in two different treatments (highcontest and low-contest competition). In both cases shape and size of the patch limited the number of group members being able to access the food. Foraging benefits were calculated from the time spent in the patch combined with the bite rate during this time. A combination and further development of such approaches would allow for even more detailed analyses. It is obvious from these studies that the location and the amount of food items are the influential setscrews that can be modified. Future studies on decisionmaking in wild animal groups should therefore incorporate experiments based on the variation of both of these variables. One possible design would be, for example, to set up a number of feeding platforms with a countable resource and systematically change the placement as well as the amount of food on each platform. In doing so, different incentives related to rank, age, sex or reproductive state, as well as differences in personality traits and cognitive abilities among the group members could be studied. If single individuals could additionally be trained to learn a certain distribution of resources, even deception and the impact of differential information level could be tested with this approach. 


\section{CHAPTER 1.3}

REACHING A CONSENSUS: TERMINOLOGY AND CONCEPTS USED IN COORDINATION AND DECISION-MAKING RESEARCH

with Andrew J. King, Cédric Sueur and Claudia Fichtel

International Journal of Primatology, Special Issue:

Group Coordination \& Decision-Making, in press (2011) 


\begin{abstract}
Research on coordination and decision-making in humans and non-human primates has increased considerably throughout the last decade. However, terminology has been used inconsistently hampering the broader integration of results from different studies. In this short article, we provide a glossary containing the central terms of coordination and decision-making research. The glossary is based on previous definitions that have been critically revised and annotated by the participants of the symposium "Where next? Coordination and decision-making in primate groups" at the XXIII congress of the International Primatological Society (IPS) in Kyoto, Japan. We discuss a number of conceptual and methodological issues and highlight consequences for their implementation. In summary, we recommend that future studies on coordination and decision-making in animal groups do not to use the terms "combined decision" and "democratic/despotic decision-making". This will avoid ambiguity as well as anthropocentric connotations. Furthermore, we demonstrate the importance of (1) taxon-specific definitions of coordination parameters (initiation, leadership, followership, termination), (2) differentiation between coordination research on individual-level process and group-level outcome, (3) analyses of collective action processes including initiation and termination and (4) operationalisation of successful group movements in the field in order to collect meaningful and comparable data across different species.
\end{abstract}

Keywords: terminology, decision-making, coordination, animal groups

\title{
1 Introduction
}

The number of studies on group coordination, leadership and decision-making in humans and non-human primates has increased considerably during the last decade (reviewed in Conradt and Roper 2005; Conradt and List 2009; King et al. 2009; Petit and Bon 2010; Fichtel et al. 2011). However, integration of results from different studies has been impeded by ambiguous terminology and conceptual/methodological shortcomings (see Jacobs 2010; Petit and Bon 2010; Pyritz et al. 2010 for recent discussions). We discussed these inconsistencies with participants during the symposium "Where next? Coordination and decision-making in primate groups" at the XXIII Congress of the International Primatological Society (IPS) in Kyoto, Japan, in September 2010. Due to the strong 
positive feedback, we decided to initiate a public discussion about these terms via an interactive online platform joined by participants of the symposium and associated researchers. The glossary contained 20 central terms regarding coordination and decision-making research, most of which were previously compiled in a comprehensive review by Conradt and Roper (2005). Each participant had the opportunity to contribute additional terms, definitions, references and commentaries. Table 1 provides the final glossary based on the summary of all entries. Additionally, we identified a number of conceptual and methodological issues that have been discussed rarely or controversially in the literature so far. In the following, we discuss the most debated terms of the glossary and a number of crucial conceptual issues in more detail.

\section{Terminology}

\subsection{Combined decisions}

Conradt and Roper (2005) defined a "combined decision" as the sum of individual group members` decisions that - unintentionally - affects the group as a whole (Table 1). However, this definition is ambiguous and cannot be separated clearly from the often used and well established term of quorum decisions in which group members also choose individually between different options until a certain threshold is reached that affects the entire group (Seeley et al. 2006; Ward et al. 2008; Table 1). In fact, the mechanisms of reaching a group decision employed in a large colony of ants (Leptothorax albipennis) choosing between several new nest sites (Franks et al. 2002) are principally the same as those employed by Tonkean macaques (Macaca tonkeana) in a pre-departure quorum (Sueur et al. 2010) or meerkats (Suricata suricatta) increasing travel speed via a vocal voting mechanism (Bousquet et al. 2010). In these species, each group member decides individually where and when to move, even if this decision is influenced by the behaviours of conspecifics. Furthermore, there is no easy way to test whether individuals aim to reach a group consensus, which was the criteria Conradt and Roper (2005) used to distinguish consensus decisions from combined decisions. As a consequence, we recommend not using the term "combined decision" in future research. 


\subsection{Democratic and despotic decisions}

The terms "democratic" and "despotic" have been used equivalent to "shared" and "unshared" in a number of recent studies on decision-making (Conradt and Roper 2003; King et al. 2008; King and Cowlishaw 2009). "Democracy" describes a consensus reached by the majority principle, whereas "despotism" characterises a consensus reached by following the choices of specific leaders in these studies. However, in primatology the term "despotic" is also used to characterise the social structure of a species, opposed to "egalitarian" or "tolerant" societies (e.g., Matsumura 1999), and the social structure of a species undoubtedly influences the process and outcome of consensus decision-making. For instance, Sueur and colleagues studied the decisions of two macaque species - with contrasting social structures - beginning a group movement following a resting period (Sueur and Petit 2008a, b). They found that egalitarian Tonkean macaques displayed an equally shared consensus whilst more despotic rhesus macaques (Macaca mulatta) displayed a partially shared consensus. These differences were attributable to the fact that in the despotic rhesus macaques, dominant individuals had a disproportionate influence on the movement decisions of group-mates: they were followed more often (than those lower-ranked individuals). In contrast, each individual in the more egalitarian Tonkean macaques had a similar influence on one another's decisions to move and the decision was equally shared. However, the social structure of a primate group can more broadly be viewed as both the cause and consequence (reinforced via a feedback loop) of social interactions such as intensity of aggression, grooming or reconciliatory patterns. These social interactions may be independent of group level decision-making processes. Thus, in order not to confound characteristics of the social system and collective decisionmaking in a group or causal relationships between these two realms, we suggest that future decision-making research should use only the terms "shared" and "unshared", and not "democratic" and "despotic".

\subsection{Leadership}

The definition of a leader should not be restricted to its spatial position during a group movement because individuals may also lead from behind, i.e. initiate and terminate a movement without being at the forefront of the group (Kummer 1968; called "hidden leadership" in Pyritz et al. 2010). Instead, a leader should be defined as an individual that 
elicits follower behaviour (from a majority of/all group members) and exerts social influence on group members either by its rank, experience, social status/connectedness or specific behaviour (King 2010; Sueur and Petit 2008b; Petit and Bon 2010). Furthermore, an individual that leads may not do so intentionally, i.e. leadership can be a passive process (King et al. 2009; King and Sueur, in press; Fischer and Zinner, this issue). For instance, in sheep (Ovis aries), individuals triggered follower behaviour by merely moving away from their group following a sound they had been trained to, thus eliciting group movements as incidental leaders (Pillot et al. 2010).

\section{Concepts and methods}

\subsection{Taxon-specific definitions}

It remains a major practical challenge for human observers to reliably identify and operationally define a group movement in the field. The most detailed definitions employed in recent studies on the coordination of group movements in different species go as follows: An individual moves a certain distance towards the edge of the group either in a defined time period (e.g., $10 \mathrm{~m}$ within 40 s) (e.g., Leca et al. 2003; Sueur and Petit 2008a, b; Stueckle and Zinner 2008), or without stopping and feeding (Bourjade et al. 2009; Ramseyer et al. 2009a, b, c), and is followed by a certain number of group members. However, even in these fairly detailed definitions, most parameters were not assessed empirically. In fact, a definition of group movements must account for a number of taxon-specific characteristics such as different travelling types (directed movements versus 'feed-as-you-go', amoeboid-like movements that do not necessarily require an initiator or coordination among group members, e.g., bonobos, Pan paniscus: Wrangham 2000), mean travel distances, ecological conditions (resource abundance, predation risk), as well as the size, composition, and cohesion of the group. Therefore, we encourage the use of operational group movement definitions for different taxa that are built upon empirical data collected prior to the study period used for the analyses of group movements. In the long term, these empirical details will generate a comprehensive database for multiple species and different contexts. Researchers will then be able to compare and contrast the results in order to see if general patterns are shared among different species. For practical details of how to operationalise definitions see Trillmich et al. (2004) and Pyritz et al. (2010). 


\subsection{Individual-level process and group-level outcome}

Studies of group coordination and decision-making examine two different levels that should be clearly distinguished. First, studies can focus on the group level, i.e. the question of whether there is consistent or variable leadership among several group movements or other collective actions (e.g., Erhart and Overdorff 1999; Jacobs et al. 2008; King et al. 2008; Pyritz et al., this issue). Second, studies can focus on the level of single movements and study the process, i.e. the question of whether decisions are shared or unshared and if they are mediated by mimetism, affiliative/genetic network relations, quorum thresholds or self-organised processes (King and Sueur, in press; Sueur and Petit 2008a, b, 2010; Sueur et al. 2009, 2010; Petit and Bon 2010). It is important to separate these levels and clearly highlight the level at which the research is focused. King and Sueur (in press) and Sueur and Deneubourg (2011) explain how a consistent leadership or a consistent order of individuals could be observed even though the decision process is shared. For example, imagine $\mathrm{n} \%$ of group members have to follow the first-moving individual (i.e. initiator) within $\mathrm{n}$ minutes for a group movement to occur, otherwise the initiator stops moving and the whole group remains stationary. A study observing movement patterns during departure would conclude that the process is 'shared' since multiple individuals contribute to the decision of the group to move. A study that only observed the order of departure when the group chose to move would observe group movements that were always led by the same individuals, the decision appears 'unshared'. Since the outcome at the group level (i.e. departure order) is easier to observe, it is no surprise that most early studies in the area of coordination focused on who leads (see King 2010 for a discussion). However, identifying who leads may not tell the whole story. For instance, an individual can always, or in most cases, be the leader of a group because his energetic reserves are always the first to be depleted (and he has to move in order to feed), but might have the same (or at least no stronger) influence on the joining process as other group members (e.g., in the case of an anonymous mimetism). In fact, a number of recent findings (e.g., macaques: Sueur and Petit 2008a, b, 2010; Sueur et al. 2009; horses: Bourjade et al. 2009) suggest shared consensus between group members at the movement level, even though a single leader or a few leaders, i.e. an unshared consensus with consistent leadership, has previously been reported (e.g., 
macaques: Reinhardt et al. 1987; horses: Feist and Mc Cullough 1976). For a further discussion of this topic see Bourjade and Sueur (2010).

\subsection{Initiation and termination of collective actions}

During the course of a group movement animals have to make two basic decisions, (a) initiation: when and in which direction to move and (b) termination: when and where to stop. Theoretically, the decision where to stop may be a second consensus decision independent from the decision during initiation and the two decisions can be initiated by the same or different leaders (Boinski 1991; Erhart and Overdorff 1999; Pyritz et al. 2010). Although this issue has been addressed rarely, it yields important implications for the decision type, i.e. there could be unshared decision-making at departure that is mitigated by individuals overtaking the initial leader and terminating the movement resulting in a shared decision-making with regard to the whole movement process. Accordingly, we suggest making the distinction between these two events, and exploring the consistency of leadership during travel. For instance, one might classify leadership as "stable", i.e. the initiator leads the movement until termination, or "unstable", i.e. the terminator is different from the initiator (Pyritz et al., this issue).

In general, it is important to study the entire process of group movements from initiation to termination in order to gain a realistic image of coordination, leadership and decision-making in a given species (Trillmich et al. 2004; Pyritz et al. 2010; Fichtel et al. 2011). Indeed, such detail will be crucial if we are to determine the temporal scale over which decisions are made, and outcomes occur. Take an example where the same individual, $X$, both initiates and terminates the movement. This could imply that (a) $X$ made the decision to move and the decision to stop, which was followed by group-mates, or (b) X made the decision to move in a particular direction of travel (e.g., Sueur et al. 2010; Noser and Byrne 2007a) in a "goal-directed fashion" toward a particular destination, and was first to arrive, but did not make the decision to terminate the movement, since all group-mates that followed were aware of the target destination at the initiation (Pyritz et al, in prep.). In other words, the decision of where to terminate had been made at the initiation. 


\section{4 "Successful" group movements}

Early studies set a threshold of $50 \%$ of group members having to follow an initiator (within a certain time frame) to consider a movement as successful (e.g., Erhart and Overdorff 1999). More recent studies considered an initiation attempt as failed only if no individual followed (Trillmich et al. 2004; Sueur and Petit, 2008a; Jacobs et al. 2008). However, the mean number of followers that determines a successful group movement is presumably taxon-specific and may change with ecological season or resource abundance (King et al. 2008; Pyritz et al., this issue). Furthermore, fission into subgroups - and an accordingly lower number of followers for single initiators - may represent the most beneficial group decision under certain conditions (Jacobs 2010; Kerth 2010). Hence, it is difficult to provide a general definition for successful or unsuccessful movements. We suggest giving a taxon- (and season-) specific threshold of success regarding followers below which the initiator does not stop and show recruitment behaviour (Table 1), or "give-up" on the movement entirely (e.g., 5 followers in chacma baboons, Papio hamadryas: Stueckle and Zinner 2008; 3 followers in Verreaux's sifakas, Propithecus verreauxis: Trillmich et al. 2004; white-faced capuchins, Cebus capucinus: Petit et al. 2009; meerkats, Suricata suricatta: Bousquet et al. 2010; Tonkean macaques: Sueur and Petit 2010). Combining this with operational group movement definitions for different taxa (above), it may be possible to uncover a common threshold - a universal - to primate group movements. In fact, examination of the studies listed above indicates that a threshold of three followers seems to be sufficient to elicit a group movement, whatever the species. This number may provide sufficient protection against predators or enough collective knowledge in order to orientate within the home range and detect resources, for example.

\section{Conclusions}

Following a public discussion about terms used in coordination and decision making research via an interactive online platform, we have come to a number of conclusions which will aid future research on this topic. First, we highlighted a number of ambiguous terms (combined decision; despotic/democratic decision) and suggested that these not be used in future coordination research. We also suggest maintaining the terms 'leader' and 'leadership', since although they can sometimes be ambiguous, as long as an 
operational definition is provided these terms are useful to broader scientific communication and integration (King 2010; Pyritz et al. 2010). Furthermore, we discussed a number of conceptual issues (group versus movement level; operationalised successful group movements; collective action process including initiation and termination). Ultimately, the implementation of our suggestions should provide comparable data on coordination and decision-making in different species and facilitate the identification of general patterns that are shared among different taxa.

\section{Acknowledgements}

We thank the participants of the symposium "Where next? Coordination and decision making in primate groups" at the XXIII Congress of the International Primatological Society (IPS) for valuable discussions and comments on the glossary and conceptual issues. Henning Lahmann kindly implemented the online glossary. We are also grateful to Joanna Setchell and the International Journal of Primatology for providing financial support and making this special issue possible. LWP is funded by the German Research Foundation (DFG: KA 1082/16-1), AJK by a NERC Postdoctoral Fellowship (NE/H016600/2) and CS by the Japan Society for the Promotion of Science. Joanna Setchell and two anonymous reviewers gave valuable comments on the manuscript. 


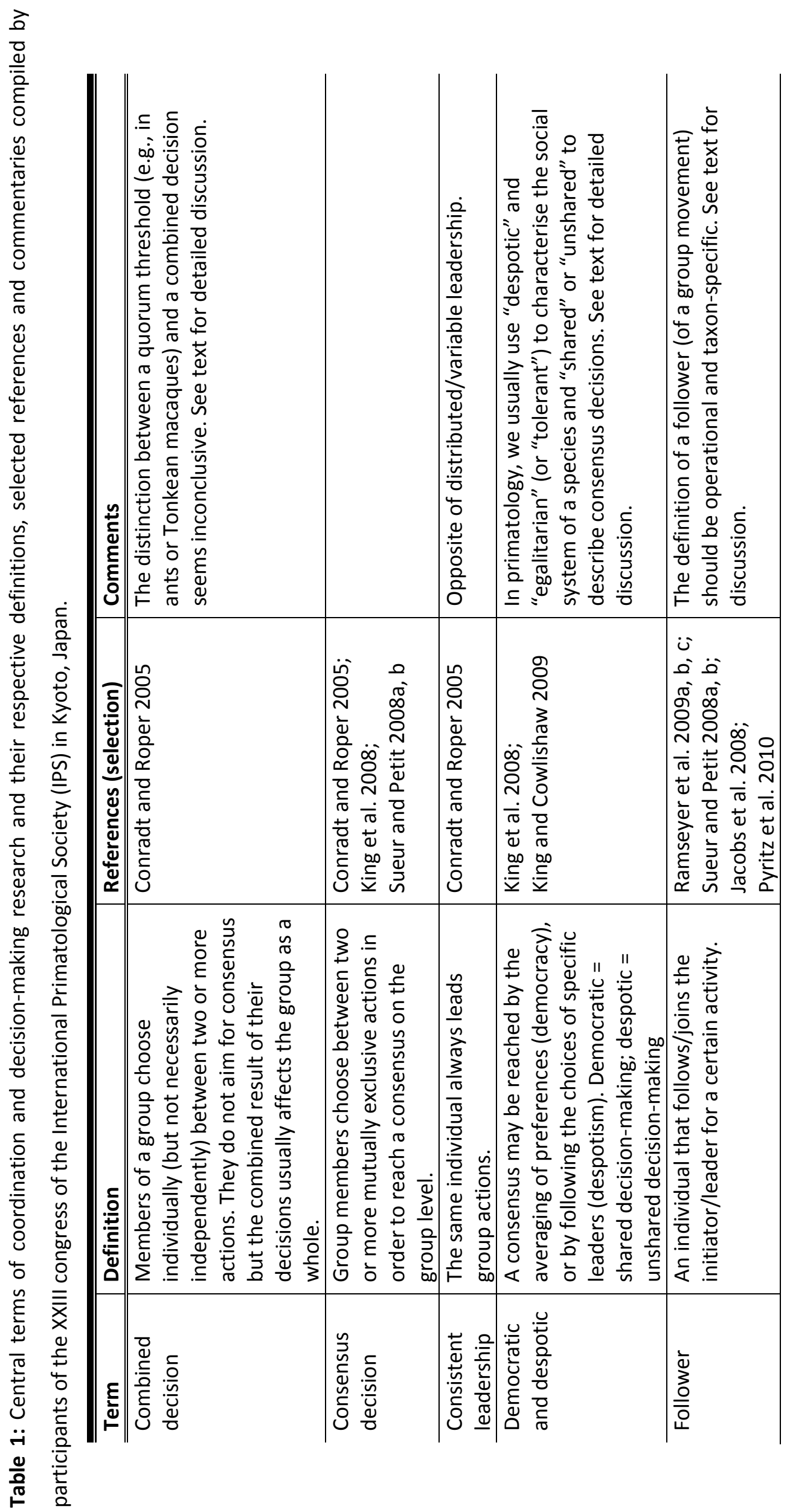




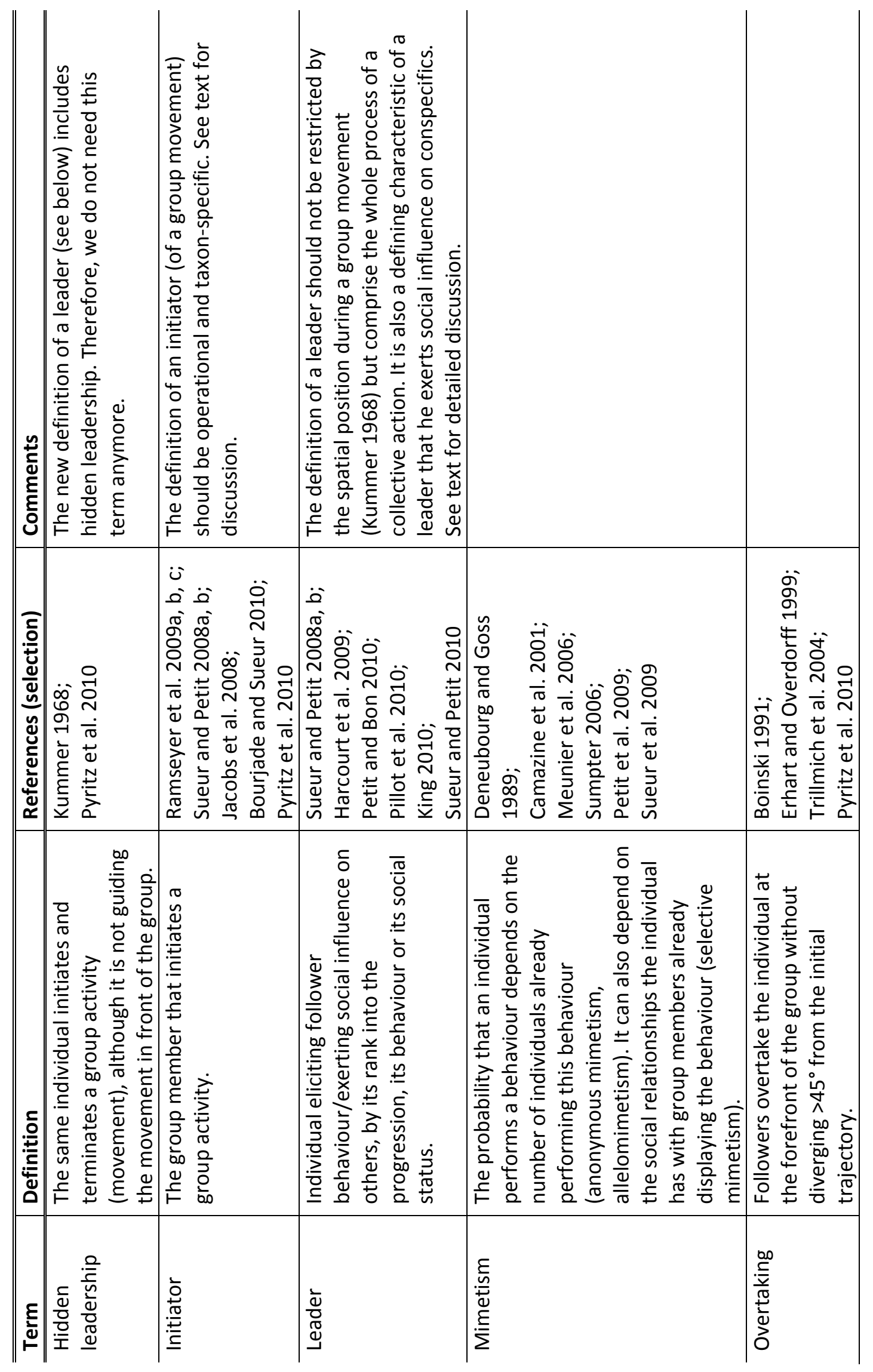




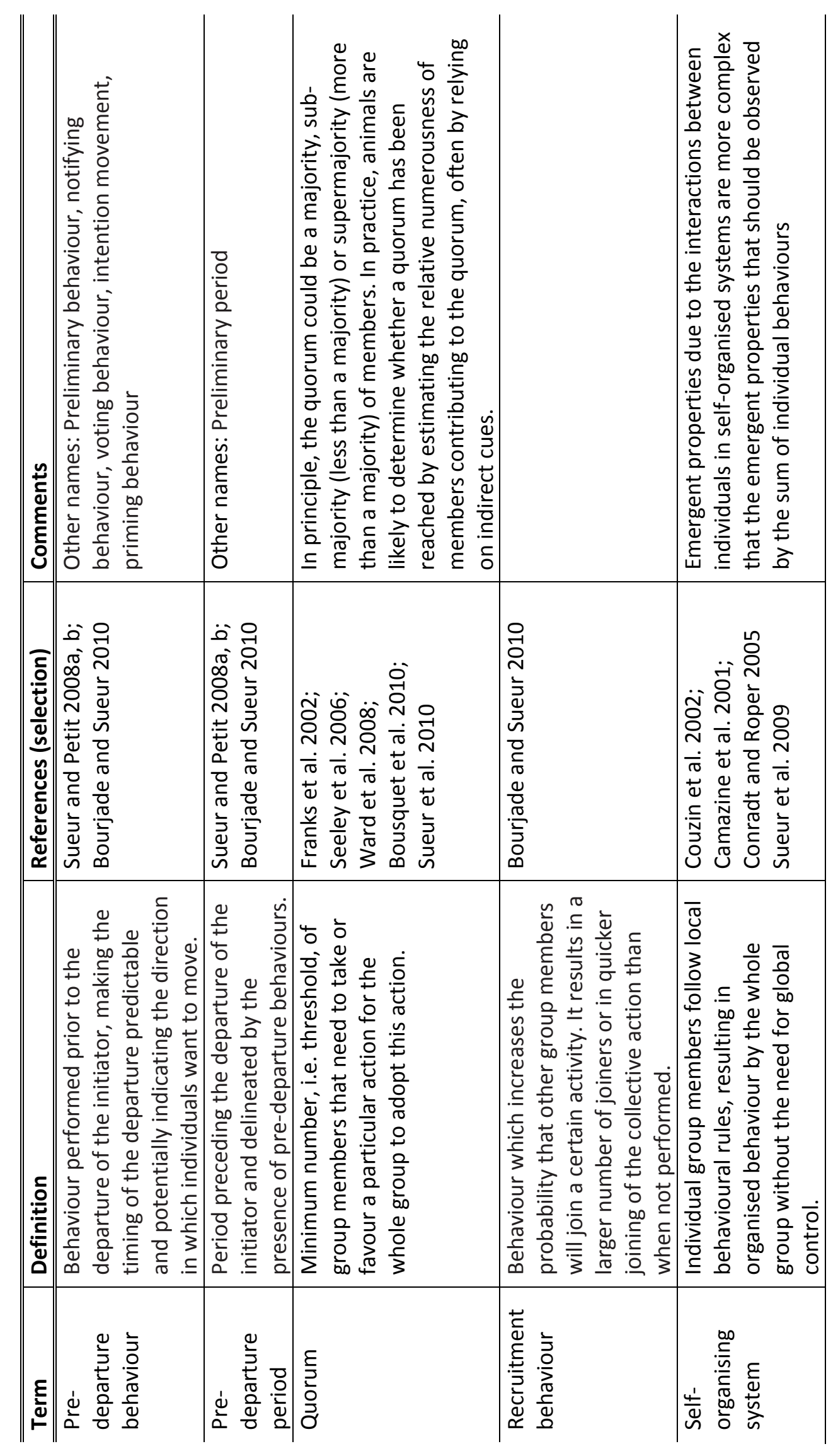




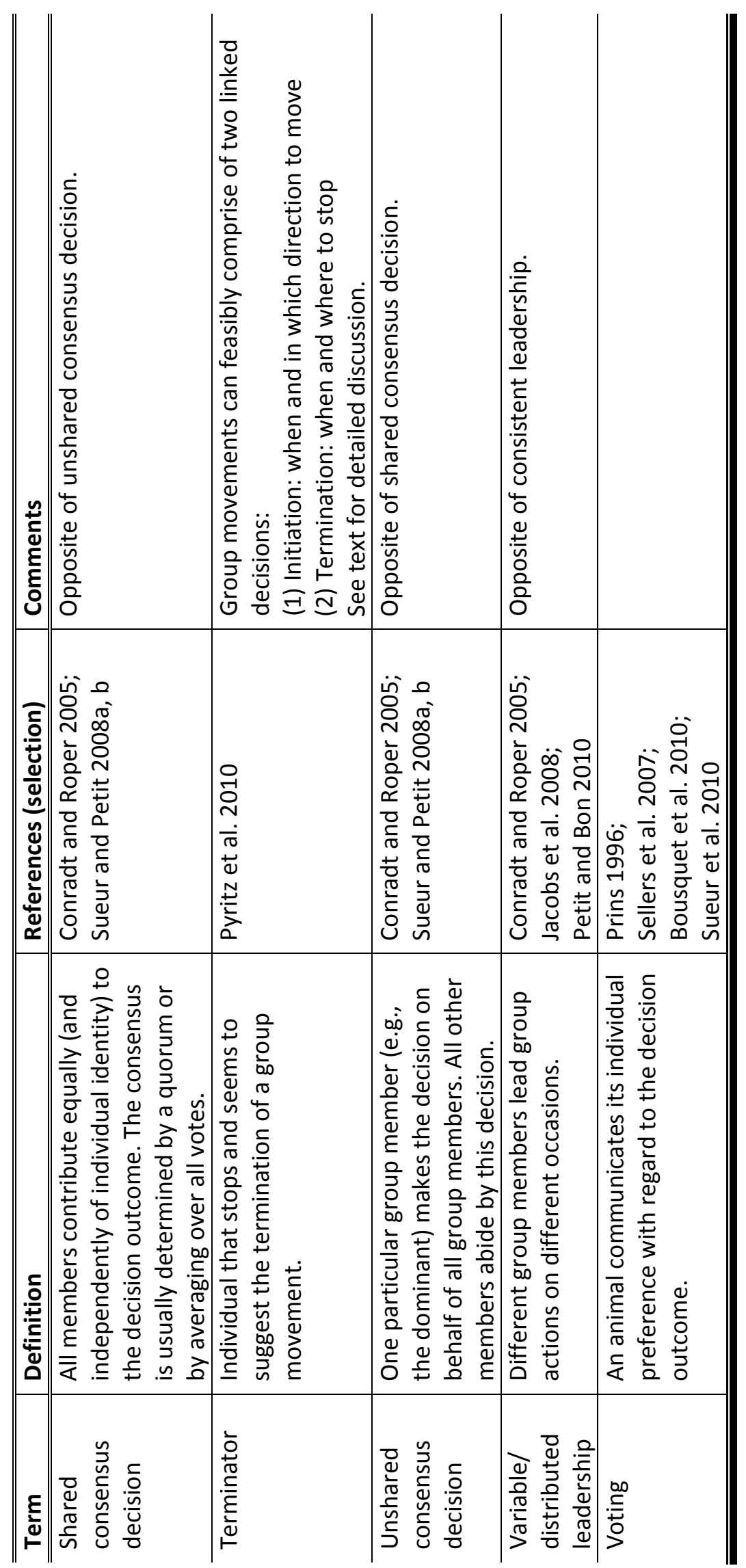




\title{
CHAPTER 2
}

\author{
DATA FROM GPS-COLLARS HIGHLIGHT EFFECTS OF \\ Sampling and Calculation Method, Climatic Seasonality and \\ Sample Size on Home Range Estimates in a Wild Primate
}

with Claudia Fichtel and Peter M. Kappeler

International Journal of Primatology: submitted 


\begin{abstract}
Accurate home range estimates are essential to address a variety of fundamental biological and conservation-related questions. However, home range estimates often differ between climatic seasons and vary with sampling method, sample size and calculation method (=estimator). Moreover, general guidelines for reporting results are lacking, which hampers comparisons among studies. The major potential of GPS collars as a method to analyze primate ranging behaviour has been stressed but most previous studies were impeded by technical difficulties. We successfully collected data with GPS collars (1 fix/30 $\mathrm{min}$ ) in four groups of red-fronted lemurs (Eulemur rufifrons) at Kirindy, Madagascar, during 6 months of field work covering two climatic seasons. Acquisition rate was high (93.86-98.92\%) and did not differ significantly between seasons despite dense leaf cover during the rainy season. In order to highlight exemplarily the effects of sampling method, estimator and climatic seasonality on home range size, we calculated differences in monthly home range estimates $(\Delta h)$ of the groups between the GPS data and simulated census data, using minimum convex polygon (MCP), adaptive (AK) and fixed kernel (FK) methods. $\Delta \mathrm{h}$ differed significantly, depending on season and estimator and was also affected by the interaction of both variables. Additionally, cumulative home range size for different estimators was calculated for the GPS data of one group. Depending on the season, different estimates reached an asymptote first. Our study demonstrates the feasibility of applying GPS collars in research on forest-living primates. However, home range sizes that we calculated varied strongly depending on several (interacting) variables. We present a number of examples from the literature to illustrate the wider implications of this finding. Finally, we make a number of suggestions to improve accuracy and comparability among future studies (choose estimator specifically for study species, study objectives and sampling parameters; report results from different estimators).
\end{abstract}

Keywords: home range size; kernel (fixed, adaptive); minimum convex polygon (MCP); red-fronted lemurs 


\section{Introduction}

Precise information on a species' home range size and ranging patterns is crucial to understanding its behavioural ecology as well as to plan conservation measures (e.g., Caro 1998; Sutherland 1998; Burgman and Fox 2003). However, there is a variety of different estimators to choose from to calculate home ranges (e.g., grid cell method: White and Garrot 1990; kernel method: Worton 1989; minimum convex polygon [MCP] method: White and Garrot 1990; Powell 2000). In fact, the choice of estimator can have dramatic effects on home range calculations (e.g., Powell 2000; Girard et al. 2002), which has been shown in recent primate studies (northern bearded saki monkeys, Chiropotes satanas chiropotes: Boyle et al. 2009; snub-nosed monkeys, Rhinopithecus bieti: Grüter et al. 2009). Furthermore, results for single estimators can differ considerably depending on a number of parameters such as sample size (Boulanger and White 1990; Seaman et al. 1999; Blundell et al. 2001), sampling effort (Swihart and Slade 1985; Girard et al. 2002; Börger et al. 2006), technical variables (smoothing parameters calculated by least-squares cross-validation: Worton 1989; Blundell et al. 2001; Gitzen and Millspaugh 2003; Hemson et al. 2005) and specific ecological factors (season: Doran et al. 1997; Li et al. 2000; Baker 2001; population density: Hoset et al. 2008).

Different estimators have different limitations regarding the above parameters. For instance, MCP estimates based on a small sample size often overestimate home range size due to high outlier sensitivity and, consequently, comprise large areas that are never frequented by the animals (Powell 2000; Burgman and Fox 2003; Grüter et al. 2009). On the other hand, MCP was more accurate than kernel estimators, which overestimated home range size for small sample sizes in a study on saki monkeys and has been suggested as the best calculation method for territorial species that regularly patrol the borders of their home ranges (Boyle et al. 2009). Kernel estimators (adaptive kernel, AK: values for smoothing parameters vary according to point concentration; fixed kernel, FK: fixed value for smoothing parameters; Worton 1989) have often been described as more accurate than MCPs (Powell 2000; Börger 2006). However, kernel methods have also been criticized in a number of studies for overestimating ranges (AK: Seaman and Powell 1996; Boyle et al. 2009; FK: Girard et al. 2002). Furthermore, the selection of smoothing factors for kernels is problematic (Worton 1989). For instance, least-squares crossvalidation often resulted in inconsistent home range estimates for small (herpetofauna: 
Row and Blouin-Demers 2006) as well as large sample sizes (Hemson et al. 2005). In summary, evaluation of different estimators is not straightforward and depends on the species as well as the specific ecological and technical variables.

Numerous new opportunities and challenges for home range estimation have been opened by the application of GPS-based technologies in the last 15 years or so and technology is advancing rapidly (Cagnacci et al. 2010; Osborne and Glew 2011). The principal advantages of these technologies comprise high spatial accuracy and reliability, high sampling rate independent of light/weather conditions or man-power, the possibility to collect data on the ranging behaviour of multiple groups simultaneously and convenient technical and statistical tools for analyses (summarized in Tomkiewicz et al. 2010). GPS collars have already been used successfully in a range of different mammal taxa (moose, Alces alces: Rempel et al. 1995; Girard et al. 2002; white-tailed deer, Odocoileus virginianus: Bowman et al. 2000; black bears, Ursus americanus: Obbard et al. 1998; brown bears, Ursus arctos: Arthur and Schwartz 1999; jaguars, Panthera onca: Cavalcanti and Gese 2009).

However, the use of GPS-collars in primatological studies has long been limited by technical difficulties, often related to the dense forest habitat of many primate species that restricts sampling success of GPS devices (e.g., Phillips et al. 1998; Dominy and Duncan 2001). In a first study using GPS collars in primates, spatial data of one adult female Japanese macaque (Macaca fuscata) were collected over a 9-day study period (Sprague et al. 2004). Total acquisition rate of fixes was $20 \%$ with almost all failures occurring in forest areas. In a second GPS study on free-ranging Japanese macaques, a total of 142 fixes with a success rate of $38 \%$ was sampled with vegetation density still negatively affecting the GPS performance (Takenoshita et al. 2005). Data logging attempts were also unsuccessful when the fixing unit was oriented downward, which happened frequently through manual manipulation of the collars by the macaques. Longer sampling periods and high percentages of successful fixes were only accomplished recently in studies on baboons at Amboseli, Kenya (Papio cynocephalus; 90 days, 99.3\%: Markham and Altmann 2008) and snub-nosed monkeys at Jinsichang, China (10 months, 82.2\%: Ren et al. 2008). In both studies, one adult individual could be fitted with a collar. However, vegetation was not a challenge in the baboon study due to open savannah habitat and 
fixing rate was rather low ( 5 fixes/day) for the snub-nosed monkeys that live in montane coniferous forest.

In this study, we do not aim to explore in detail the best estimator for a given sampling method, sample size and season for our study species (red-fronted lemurs, Eulemur rufifrons, at Kirindy Forest, western Madagascar). Instead, our approach is supposed to highlight a number of chances and pitfalls in current research on primate ranging behaviour in general. First, we demonstrate the feasibility of applying custommade GPS collars to collect continuous spatial data of several groups of a forest-living primate over a prolonged period of time throughout different climatic seasons (rainy, dry). Second, we compare the effects of different estimators (AK, FK, MCP) and climatic seasonality on home range estimates on the basis of different sampling methods, i.e. GPS collar data as a reliable basis and a subset of these data simulating a minimal daily census. Third, we explore the effect of sample size on cumulative home range estimates for the GPS data of one group over different seasons. Finally, we place our results in a wider framework by providing comparisons with other studies on primate ranging behaviour and stress a number of implications that could improve accuracy and comparability of future studies in this context.

\section{Materials and Methods}

\subsection{Study site}

We collected data at the field station of the German Primate Center (DPZ) at Kirindy Forest, a dry deciduous forest located at $44^{\circ} 39^{`} \mathrm{E}$ and $20^{\circ} 03^{\prime} \mathrm{S}$ about $60 \mathrm{~km}$ north of Morondava, western Madagascar (Sorg et al. 2003). The site is managed within a forestry concession operated by the Centre National de Formation, d'Etudes et de Recherche en Environnement et Foresterie (CNFEREF), Morondava. The forest is characterized by a pronounced seasonality with a hot rainy season between December and April and a cooler dry season between May and November (Sorg and Rohner 1996), during which most trees shed their leaves and only a couple of small water holes remain in the bed of the Kirindy river (Scholz and Kappeler 2004). Red-fronted lemurs live in multi-male, multifemale groups comprising 5-12 individuals (Overdorff et al. 1999). Long-term research on several groups of red-fronted lemurs has been ongoing at Kirindy since 1996 (e.g., Wimmer and Kappeler 2002; Ostner and Kappeler 2004). 


\subsection{Study animals and data collection}

We followed four habituated groups (individuals per group during the study period: A: 78; B: 8-10; F: 8-9; J: 6-7) of individually marked red-fronted lemurs inhabiting a 60 ha study area within Kirindy Forest and observed them on a daily basis between March-May and September-November 2008 with one or two researchers, respectively, as part of a comprehensive study on group coordination processes (Pyritz et al. in prep.). We conducted observations between approx. 7:00-10:00h and 14:00-17:00h each day, and each group was observed every second day, alternating between the morning and the afternoon, respectively. In mid-March and mid-September, we fitted one adult male per group with a custom-made GPS-RF collar (for system specification see Kümmeth and Heidrich 2007; Fig. 1) during brief anaesthesia induced by applying $0.2 \mathrm{ml} \mathrm{GMII}$ (Rensing 1999), following blow-pipe darting by an experienced Malagasy technician. This happened also within the framework of the coordination study, i.e. we collected spatial data not only for home range estimates but also to study group encounters and small-scale movement patterns.

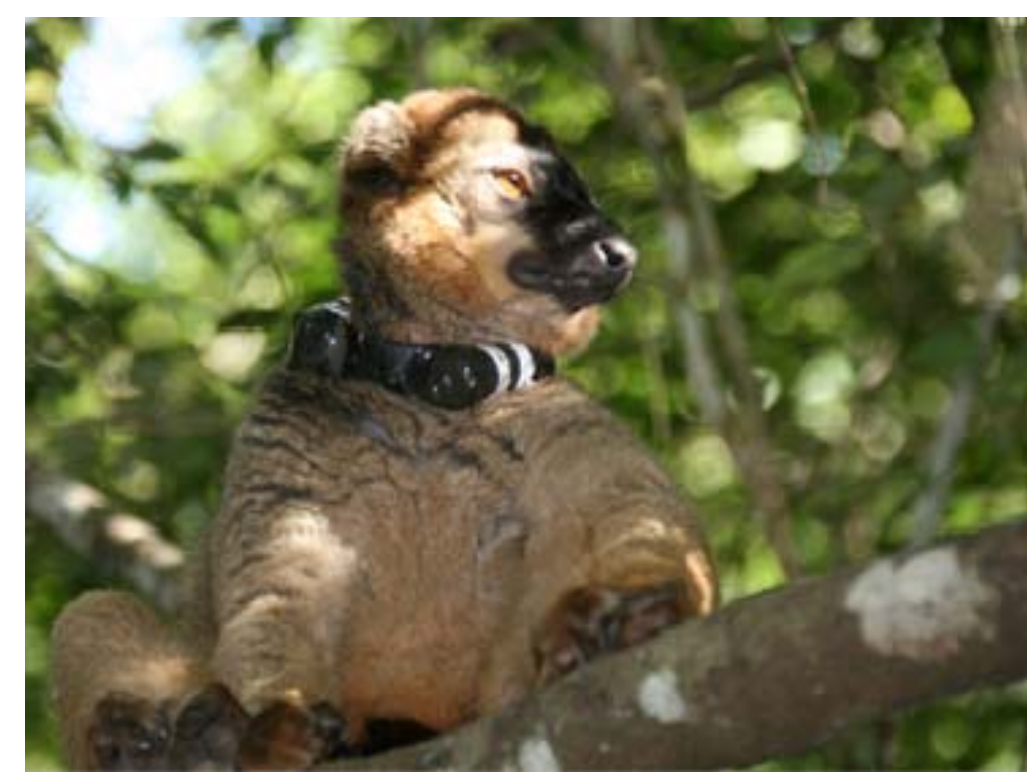

Fig. 1: Red-fronted lemur adult male at Kirindy forest carrying a GPS collar (photo by LP).

The collars consisted of a GPS-fixing unit ( $39 \mathrm{~mm} \times 22 \mathrm{~mm} \times 14 \mathrm{~mm}$ ) and a battery affixed to a soft neck collar with a waterproof coating and had a total mass of $49 \mathrm{~g}(=2.8 \%$ of average body mass). The battery ( $27 \mathrm{~g}$ ) was heavier than the fixing unit (13 g), therefore, fixing units were automatically oriented upward on the neck of the animals. 
The collars logged the spatial position of the animals every 30 min for a continuous time period of approx. 2.5 months (Table 1). We could download data via a radio link from a distance between 3-30 $\mathrm{m}$ in the forest, depending on leaf cover density, using a handheld base station (Kümmeth and Heidrich 2007).

Anaesthetized individuals recovered within two hours following darting and were returned to their social groups. We did not observe any injuries or adverse effects on behaviour as a result of the capture procedure or the GPS collars, i.e. collared individuals were foraging, resting, travelling and engaging in social interactions with their group members like before. In groups B and F, we fitted the same male with a GPS collar in both study periods. In groups A and J, we were not able to capture the same males again in September and collared two other males of the respective groups instead. However, the small groups of red-fronted lemurs at Kirindy are highly cohesive (Pyritz et al. 2010; Pyritz et al., in prep.). Hence, we assumed the GPS data based on movements of any group member to provide a realistic proxy of the movement patterns of the entire group. All GPS collars were removed within six months after the batteries expired. Our research was approved by the Malagasy Ministère de l'Environnement et des Eaux et Forêts.

Table 1: Total logging periods and performances of the eight GPS collars used in the study.

\begin{tabular}{|c|c|c|c|c|c|}
\hline Season & Group & Logging time period & $\begin{array}{l}\text { Total no. of } \\
\text { logged } \\
\text { GPS points }\end{array}$ & $\begin{array}{l}\text { Total no. } \\
\text { of failed } \\
\text { logging events }\end{array}$ & $\begin{array}{l}\text { Percentage } \\
\text { of failed } \\
\text { logging events }\end{array}$ \\
\hline \multirow{4}{*}{ Rainy } & $A$ & Mar 18 - May 28, 2008 & 3422 & 37 & 2.80 \\
\hline & $B$ & Mar 18 - May 28, 2008 & 3424 & 96 & 1.08 \\
\hline & $\mathrm{F}$ & Mar 18 - May 28, 2008 & 3416 & 86 & 2.52 \\
\hline & $\mathrm{J}$ & Mar 18 - Jun 2, 2008 & 3700 & 54 & 1.46 \\
\hline \multirow{4}{*}{ Dry } & $A$ & Sep 15 - Dec 2, 2008 & 3716 & 104 & 2.79 \\
\hline & $B$ & Sep 15 - Nov 30, 2008 & 3692 & 103 & 2.80 \\
\hline & $\mathrm{F}$ & Sep 15 - Nov 26, 2008 & 3494 & 114 & 3.26 \\
\hline & $\mathrm{J}$ & Sep $16-\operatorname{Dec} 3,2008$ & 3781 & 232 & 6.14 \\
\hline
\end{tabular}




\subsection{Data analyses}

We assessed general performance of the GPS collars via the percentage of failed logging events from the original data sets (Table 1) and calculated an exact Wilcoxon matchedpairs signed rank test in order to compare the performance of the devices between the rainy and the dry season using PASW Statistics 18 (SPSS Inc., Chicago, IL, 2009). Since the collars were activated slightly time-shifted in mid-March and mid-September, respectively, and ran for slightly different time spans in each group (approx. 75 days), we generated six statistical months for further computations of home ranges, each comprising 18.5 days during which the loggers for all groups were active (March: Mar 20 Apr 08, 2008; April: Apr 10 - Apr 28, 2008; May: May 01 - May 19, 2008; September: Sep 17 - Oct 05, 2008; October: Oct 08 - Oct 26, 2008; November: Oct 29 - Nov 16, 2008). The impact of autocorrelation on home range estimates has long been discussed controversially (e.g., Swihart and Slade 1985, 1997; Blundell et al. 2001) and is context-, scale- and system-dependent (Fortin and Dale 2009). As the focus of this study was on the effects of different estimators, sampling methods and seasonality, rather than small-scale movement patterns, only points logged at $2 \mathrm{~h}$ intervals - red-fronted lemurs rarely stay resting or feeding at the same place for $>60 \mathrm{~min}$ - were included in the home range estimates for the collar data to avoid potential autocorrelation effects ( 12 points/day = 222 points/statistical month). For the census data, we used a subset of the collar data (1 point/day taken between 6-8 am), resulting in 19 points per statistical month. We are well aware that this is a minimal daily census and that most primatologists base their home range calculations on much larger daily samples (e.g., 120 points/day: Boyle et al. 2009). However, there is also a number of recent studies reporting home range estimates based on small sample sizes (e.g., average of 3 points/day: Li et al. 2000; 5 points/day: Ren et al. 2008; 40-124 points/month [average=3 points/day]: Grueter et al. 2009). In general, we want to highlight exemplarily the impact of sampling method on home range estimates rather than providing realistic ranging data for our study species. Given this target, we feel that it is justified to sharpen contrasts by using minimal daily census data.

We estimated home range sizes for GPS collar and census data using adaptive kernel (AK), fixed kernel (FK) and minimum convex polygon (MCP) methods. Estimates from the kernel methods were taken at the $90 \%$ values (e.g., Boyle et al. 2009). MCP estimates were calculated with $90 \%$ (10\% outlier removal; MCP90) and $100 \%$ of fixed 
locations (MCP100), respectively. MCP90 is usually not used as a standard calculation method. Nonetheless, we included it in our calculations because MCP has often been reported to overestimate home range size due to high outlier sensitivity (e.g., Burgman and Fox 2003). By removing the outer $10 \%$ of locations, we aimed to broadly reduce this effect (see also Worton 1995b). All spatial computations were conducted using Home Range Extension (Rodgers and Carr 2002) for ArcView GIS 3.3 (Environmental System Research Institute [ESRI] 2007). We did not use least-squares cross-validation to assess kernel smoothing factors (Worton 1995a) because several studies reported inconsistent results (Hemson et al. 2005; Row and Blouin-Demers 2006) or excessive fragmentation of home ranges (Blundell et al. 2001).

In order to analyze effects of estimator and season on home range estimates against the background of different sampling methods (collar versus census), we assessed square root-transformed (sqrt; in order to normalize the data) differences in home range size $(\Delta h)$ between GPS collar and census data for each group $(A, B, F, J)$, each study month (March, April, May, September, October, November) and each estimator (AK, FK, MCP90, MCP100):

sqrt $\Delta h$ (group, month, estimator) $=\left|h_{\text {collar }}-h_{\text {census }}\right|$

Months were then grouped into two seasons ([end of] rainy season: March-May; [end of] dry season: September-November) and we calculated a linear mixed model (LMM; Zuur et al. 2009) with estimator, season and the interaction of estimator and season as fixed factors and group as random factor. We chose sqrt $\Delta \mathrm{h}$ as response variable in order to reduce the number of variables in the model. Otherwise, we would have had to include sampling method (collar or census) as additional fixed factor. Furthermore, it facilitates graphical interpretation of the results because it reduces the number of boxplots in Fig. 2 . Models were fitted using R software (R Development Core Team, Vienna, Austria, 2010) with the Ime4-package. We used Akaike's Information Criterion (Akaike 1974) to remove parameters in a step-wise fashion in order to select the most parsimonious model with the best fit. Factors were excluded only if this improved the model fit by $>2$ AIC units (Mundry and Nunn 2009). We used maximum likelihood ratio tests to test whether a fixed factor explained a significant amount of the variance in the presence of the other fixed faxtors and to test the final model with fixed factors against the null model including only the random factors (Faraway 2006). As there was high variance in the data of the four 
different groups, we also calculated a LMM with the same factors but $25 \%$ of the outliers removed to improve the data distribution for the model fitting. However, results were qualitatively the same for both data sets. Therefore, we report the statistics of the LMM incorporating all data but used the outlier-free data set for graphic implementation in order to improve the facility of inspection (Fig. 2).

In order to analyze the effect of sample size, we calculated cumulative home range estimates for the GPS collar data of one group (F). The data set was again split into two parts according to season (rainy season: March-April; dry season: October-November). Home ranges were calculated from 0 to 444 points (=2 months) for each season using 12point increments ( $=1$ day) to compare the development of single estimates and asymptote thresholds under different ecological conditions. We generated all graphs using PASW Statistics 18 and the significance level for all tests was set at 0.05 .

\section{Results}

\subsection{Performance of GPS collars}

During the entire study period, sampling success of the eight GPS collars was high. The devices did not miss more than six points, the equivalent of $3 \mathrm{~h}$, consecutively. The percentage of failed logging events was generally low and ranged between 1.08-6.14\% (Table 1). On average, failed events were slightly higher during the dry season from September to November (mean values \pm SD: $3.75 \pm 1.61 \%$ ) than during the rainy season from March to May $(1.97 \pm 0.83 \%)$ but the difference was not significant (exact Wilcoxon matched-pairs signed rank test: $Z=-1.461, p=0.144)$. Data download in the forest was unproblematic, even if data had not been downloaded for periods of up to 10 days, and downloading took up to $13 \mathrm{~min}$ using the handheld base station. During our observations, we never observed an animal manipulating the collars and the fixing unit was always oriented upward.

\subsection{Home range estimates}

Effects of sampling method, estimator and season

Our aim was to highlight effects of estimator, season and sampling method (collar versus census) on home range estimates. Home range sizes of single groups varied considerably with sampling method, estimator and season and ranged between 3.6 ha and 99.1 ha 
(overall mean=24.1 \pm 16.9 ha; Table 2). Estimates based on census data were smaller than estimates based on GPS collar data in the rainy (mean $_{\text {collar }}=18.3 \pm 5.4$ ha; mean $_{\text {census }}=16.4 \pm 9.8$ ha) but larger in the dry season $\left(\right.$ mean $_{\text {collar }}=28.3 \pm 12.5$ ha; mean $_{\text {census }}=33.2 \pm 26.2 \mathrm{ha}$ ) with standard deviations being larger for census than GPS collar estimates in both seasons. MCP90 methods consistently provided the smallest estimates for all groups, sampling methods and seasons. AK and MCP100 methods resulted in the largest estimates for GPS collar data in equal measure, whereas AK estimates were consistently the largest for the census data, except for one case (FK; Table 2). On average, home range estimates were larger for the dry (30.8 $20.6 \mathrm{ha})$ than for the rainy season (17.3 \pm 7.9 ha).

Table 2: Selected home range sizes of the four study groups calculated for different sampling methods, analytical methods and seasons.

\begin{tabular}{l|l|l|l|l|l}
\hline \hline \multirow{2}{*}{ Season } & \multirow{2}{*}{ Group } & \multicolumn{2}{|l|}{ Collar data } & \multicolumn{2}{l}{ Census data } \\
\cline { 3 - 6 } & & $\begin{array}{l}\text { Min estimate } \\
\text { (ha) }\end{array}$ & $\begin{array}{l}\text { Max estimate } \\
\text { (ha) }\end{array}$ & $\begin{array}{l}\text { Min estimate } \\
\text { (ha) }\end{array}$ & $\begin{array}{l}\text { Max estimate } \\
\text { (ha) }\end{array}$ \\
\hline \hline \multirow{5}{*}{ Rainy } & A & MCP90 (15.1) & AK (23.3) & MCP90 (4.4) & AK (30.7) \\
\cline { 2 - 6 } & B & MCP90 (8.9) & AK (22.4) & MCP90 (3.6) & AK (33.2) \\
\cline { 2 - 6 } & F & MCP90 (10.8) & MCP100 (40.5) & MCP90 (5.9) & AK (36.3) \\
\cline { 2 - 6 } & J A & MCP90 (10.1) & MCP100 (25.3) & MCP90 (5.2) & FK (35.3) \\
\cline { 2 - 6 } Dry & B & MCP90 (12.2) & MCP100 (20.7) & MCP90 (5.5) & AK (23.6) \\
\cline { 2 - 6 } & F & MCP90 (23.0) & MCP100 (53.0) & MCP90 (20.1) & AK (86.8) \\
\cline { 2 - 6 } & J & MCP90 (11.5) & MCP100 (66.1) & MCP90 (5.9) & AK (99.1) \\
\hline \hline
\end{tabular}

AK: Adaptive kernel (at 90\% values); FK: Fixed kernel (at 90\% values); MCP: Minimum convex polygon (at 90 and $100 \%$ values, respectively).

The LMM revealed significant effects for estimator (estimate: 0.93, SE: 1.01, $p<0.05$ ), season (estimate: $2.49, \mathrm{SE}: 0.63, \mathrm{p}<0.001$ ) and the interaction of estimator and season (estimate: $-0.62, \mathrm{SE}: 0.23, \mathrm{p}<0.05$ ) on differences in home range estimates between the two different sampling methods (sqrt $\Delta h$ ). The final model comprising these three factors had a significantly better fit than the null model $\left(n=96 ; \chi^{2}=18.32, d f=3\right.$, $p<0.001$; intercept: estimate: $-0.71, \mathrm{SE}: 1.01, \mathrm{p}=0.50)$. In the rainy season, $\Delta \mathrm{h}$ was largest for MCP100 (3.09 \pm 0.56$)$, however, variation among all estimators was generally small 


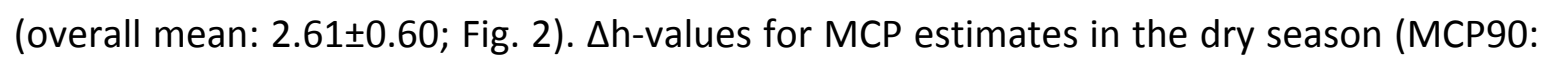
2.52 \pm 0.40 ; MCP100: $3.24 \pm 0.52$ ) were similar to those during the rainy season (MCP90: 2.34 $\pm 0.66 ;$ MCP100: 3.09 \pm 0.56$)$. Contrarily, $\Delta \mathrm{h}$ was much larger for dry-season kernel

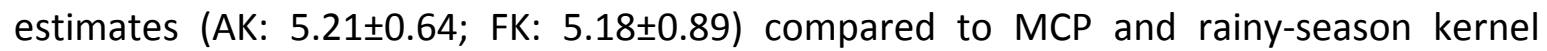
estimates (AK: 2.63 \pm 0.43 ; FK: 2.34 \pm 0.34 ; Fig. 2). Dispersion of $\Delta$ h was higher for kernel than MCP estimates during the dry season whereas the opposite pattern was found for the rainy season.

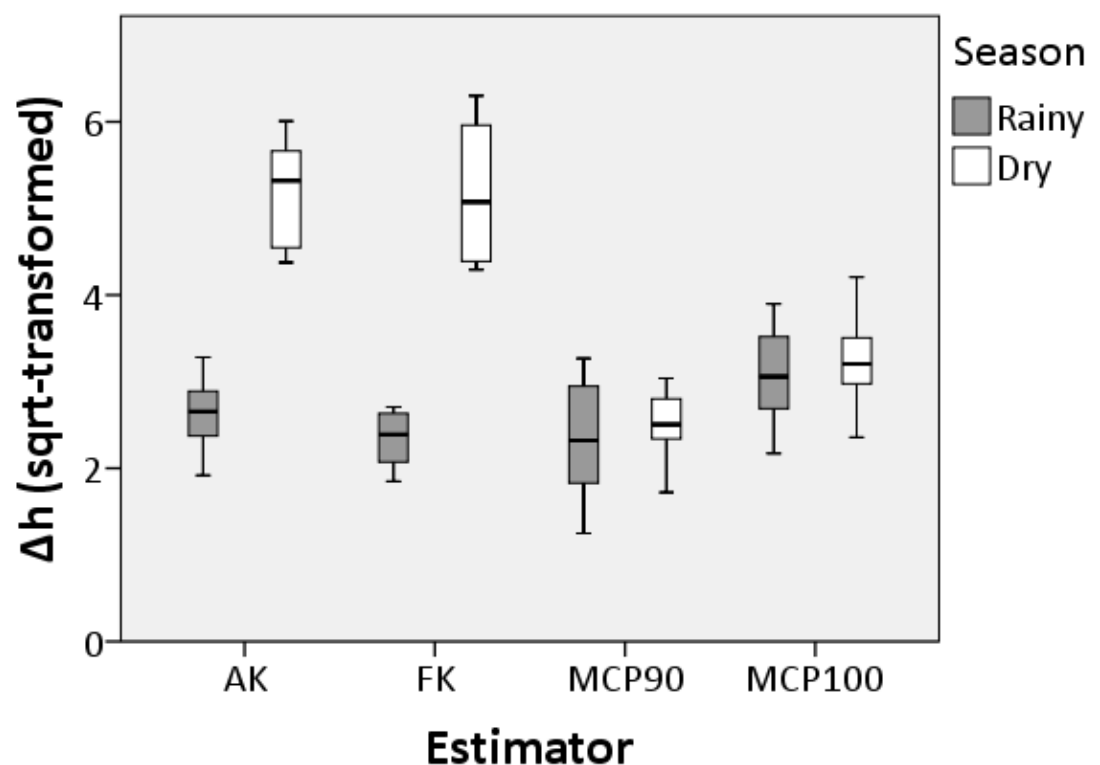

Fig. 2: Square root-transformed differences in home range size between GPS collar and census data $(\Delta \mathrm{h})$ for four groups of red-fronted lemurs varied significantly with estimator (AK, FK, MCP90, MCP100) and season (rainy, dry). Shown are medians, 25-75\% quartiles (box) and ranges (whiskers). AK: Adaptive kernel; FK: Fixed kernel; MCP90: Maximum convex polygon at 90\% values; MCP100: Maximum convex polygon at $100 \%$ values

\section{Effects of sample size and season}

We wanted to show how different estimators perform with increasing sample size and, thus, how many data points are needed for stable results of different calculation methods. Cumulative home range size varied strongly between the two seasons and was larger for each estimator in the dry season (Fig. 3a, b). MCP100 methods consistently provided the largest estimate, differing by 14.3 ha (25.7\%) between seasons. In contrast, MCP90 estimates were the smallest in both seasons with a difference of 15.7 ha (52.5\%). $A K$ and FK methods provided intermediate home range sizes (seasonal difference for AK: 
23.3 ha, i.e. 52.4\%; seasonal difference for FK: 21.1, i.e. 51.3\%) with AK estimates being slightly larger in both seasons (rainy: 1.2 ha, 5.7\%; dry: 3.3 ha, 7.5\%).
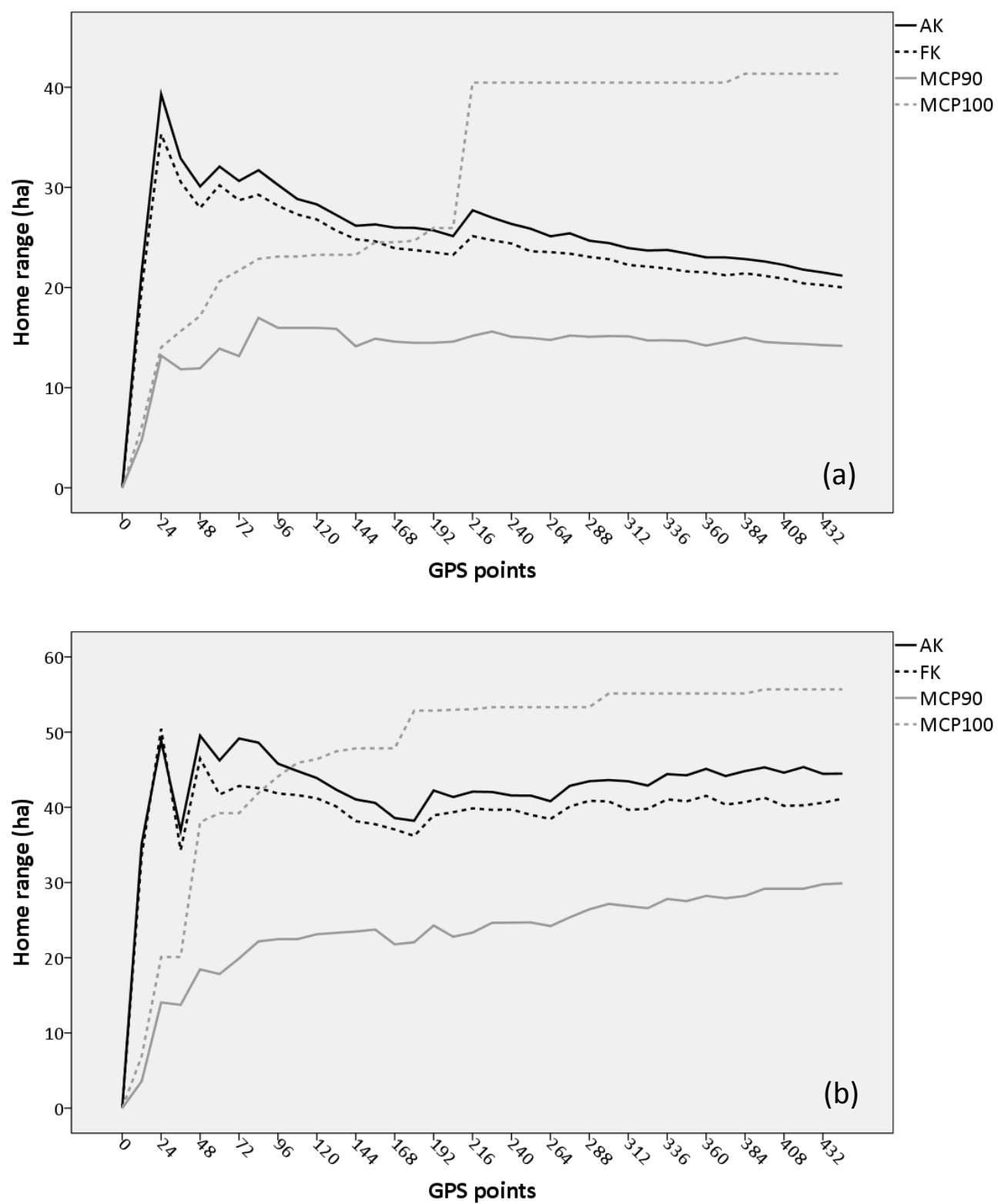

Fig. 3: Home range size varied depending on analytical method and cumulative number of GPS points. Shown are collar data of one group (F) during the rainy season (a) and during the dry season (b), respectively.

MCP100 estimates reached an asymptote at 384 (=32 days; rainy season) and 300 points (=25 days; dry season), respectively (Fig. 3a, b). However, fluctuations in estimates were only minor once 168 points (=14 days; rainy season) and 216 points (=18 days; dry season), respectively, were exceeded. MCP90 estimates levelled off at a low sample size of 144 points (=12 days) in the rainy season, while in the dry season the respective curve 
kept increasing until the final sample size of 444 points (=37 days) was reached. Sample size had to be larger to reach an asymptote for both kernel estimates in the rainy (>444 points) than in the dry season (AK: 336 points=28 days; FK: 276 points=23 days). Within single seasons, MCP90 estimates were the smallest throughout. Kernel methods produced larger estimates than MCP100 until an average of 176 points (=15 days) was exceeded in the rainy season (Fig. 3a) and an average of 102 points (=9 days) was exceeded in the dry season (Fig. 3b), respectively. AK estimates were on average $7.3 \%$ (rainy season) and 7.0\% (dry season) higher than FK estimates.

\section{Discussion}

\subsection{Performance of GPS collars}

Collars collected locations in $93.9-98.9 \%$ of logging attempts, which is a high sampling success compared to recent studies on other forest-living mammals (black bears, $32-65 \%$ : Obbard et al. 1998; white-tailed deer, 85\%: Bowman et al. 2000) and on performances of GPS collars tested under varying canopy conditions (75\%: Phillips et al. 1998; 93-98\%: Di Orio et al. 2003). Compared to other studies on forest-living primate species, we also achieved high acquisition rates (Japanese macaques, 20\%: Sprague et al. 2004; 38\%: Takenoshita et al. 2005; snub-nosed monkeys, 82.2\%: Ren et al. 2008). In fact, it is close to the acquisition rate accomplished in open savannah habitat (baboons, $99.3 \%$ : Markham and Altmann 2008). Performance did not differ significantly between seasons, i.e. dense foliage cover during the rainy season at our study site did not negatively affect acquisition rate, which is a contrast with previous studies (Sprague et al. 2004; Takenoshita et al. 2005). Hence, our results show that reliable ranging data of forest-living primates can be collected over several months using modern GPS collars. However, our study species did not bite or manually manipulate the loggers, i.e. they were not damaged, and the fixing unit was always oriented upward, which may not always be the case in other, more manipulative species (see, e.g., Takenoshita et al. 2005).

\subsection{Red-fronted lemur home range estimates}

\section{Effects of sampling method, estimator and season}

Mean home range size calculated in this study $(24.1 \pm 16.9$ ha) falls between those reported for two other sites in Madagascar that are characterized by clearly different 
habitat parameters (dry deciduous forest at Antserananomby: 0.75-1 ha [locations plotted on prepared maps], Sussman 1975; rain forest at Ranomafana: 95-100 ha [MCP], Overdorff 1993b). This strong geographical variation demonstrates the general impact of habitat quality on home range size. However, home range size at Kirindy also varied considerably with sampling method, estimator and season (Table 2). First, census estimates had larger standard deviations than estimates from GPS collars throughout, i.e. results were more variable. Presumably, the small sample size for census data and corresponding higher susceptibility for outliers produced this effect (e.g., Seaman et al. 1999; Boyle et al. 2009). Second, there was variation in the estimator providing the largest home range size but MCP90 estimates were the smallest throughout, indicating consistent underestimation due to inadequately high and biologically arbitrary outlier removal (White and Garrott 1990). In fact, for studies without a pure conceptual focus like ours, outlier removal should be handled carefully, be based on thorough inspection of the mapped data set and, if possible, be matched with parallel behavioural observations (Grüter et al. 2009). Third, home range size was on average larger during the dry season. This effect is presumably due to the behaviour of two groups $(B, F)$ that had to make long excursions to reach remaining water holes in the dry season on a daily basis, resulting in increased use of areas usually not frequented in the rainy season (Scholz and Kappeler 2004).

Our results showed not only single factors to affect home range estimates but the interaction of estimator and season was significant, too. In the rainy season, variation in $\Delta \mathrm{h}$ among estimators was rather small. Furthermore, differences between GPS collar and census data for MCP estimates were similar between seasons. However, $\Delta \mathrm{h}$ was much larger for kernel estimators in the dry season compared to all other calculation methods. Thus, MCP methods seemed to be more robust towards varying sampling methods and seasons than kernel methods in our study (Seaman and Powell 1996; Girard et al. 2002). On the other hand, the pronounced seasonal differences among the kernel estimators and their large range for $\Delta h$ during the dry season seem to better reflect the seasonal migrations to ephemeral water holes by some groups mentioned above (Scholz and Kappeler 2004). Hence, our results highlight the importance of analysing long-term spatial data using different estimators in order to reveal seasonal ranging patterns. 


\section{Effects of sample size and season}

MCP90 estimates levelled off first in the rainy season, whereas asymptotes were reached first for kernel and MCP100 methods in the dry season. Therefore, the most stable estimator for our data seemed to be season-dependent. However, MCP90 estimates were again the smallest throughout, presumably due to inadequate outlier removal (see above). Hence, kernel methods may also have provided the most accurate estimates for the rainy season and, therefore, be the more reliable choice regarding our study. In general, our findings concerning the minimum number of locations needed to reach an asymptote for home range estimates are in accordance with a study on moose, suggesting that $30-100$ locations seasonally and $100-300$ points annually were sufficient (Girard et al. 2002). In contrast, Boyle et al. (2009) demonstrated that at least 390 points (AK), 720 points (FK) and 760 points (MCP100), respectively, were needed to reach stable estimates for bearded saki monkeys. However, in this study daily increments of 120 randomly selected points ( $=1 \mathrm{fix} / 5 \mathrm{~min}$ over $10 \mathrm{~h}$ ) were used, therefore, the results are not directly comparable. Therefore, it appears that the minimum number of required locations to obtain a reliable estimate depends on sampling intervals as well as specific characteristics of the study species and its habitat.

\subsection{General constraints and future implications}

The main objective of this article is not to discuss in detail the best estimator for a given sampling method, sample size and season for our study species. Instead, we set out to highlight influential variables, constraints of current approaches and consequences for future studies from a general perspective. With regard to this objective, our results show that (in seasonal habitats) home range size strongly depends on climatic seasonality and a number of analytical parameters (sampling method, estimator and sample size). Furthermore, different variables may interact with each other, e.g., climatic season and estimator. Hence, the same variable might have different impacts on home range estimates depending on the time of data collection. Similar results were also generated in a study on snub-nosed monkeys (Grüter et al. 2009). In this case, MCP methods yielded more realistic estimates for monthly and seasonal home ranges. However, for estimates of total or annual home ranges, the grid cell method provided the most precise calculations. 


\section{Calculation methods and comparability among studies}

Many studies report home range size from one estimator only, which often makes it difficult to compare results and disentangle effects of different calculation methods among studies (Harris et al. 1990). For instance, in two recent studies on sportive lemurs, home range area for red-tailed sportive lemurs (Lepilemur ruficaudatus) and the only slightly smaller Milne-Edward's sportive lemurs (Lepilemur edwardsi) were calculated using kernel analysis (Zinner et al. 2003) and MCP analysis (Warren and Crompton 1997), respectively. Furthermore, sampling regimes were different within and between studies. Some of the home ranges calculated by Zinner et al. (2003) are based on sample sizes $<50$ points, others on sample sizes $>100$ points collected in different study periods between 1995 and 2001. Warren and Crompton (1997) conducted at least 4 all-night follows (=12 h) for each individual over an 18-month study period collecting 144 points/night on which home range estimates are based.

The mean MCP estimate for the Milne-Edward's sportive lemurs (1.1 ha) was larger than the mean kernel estimate for the red-tailed sportive lemurs ( $0.8 \mathrm{ha})$. These differences in home range size for the sister taxa could be due to ecological factors: the studies were conducted in two forests in western Madagascar that differ in various ecological factors (Sorg and Rohner 1996; Schmelting et al. 2000; Ganzhorn and Sorg 1996; Ganzhorn et al. 1999). However, home range size estimates for L. edwardsi may also be larger because MCP methods often result in overestimation (Powell 2000; Burgman and Fox 2003; Grüter et al. 2009). Alternatively, sampling regimes could be responsible for the difference. In fact, Warren and Crompton (1997) reported that field conditions hampered statistical sampling of seasonality in their study, i.e. data were mainly collected in the dry season.

\section{Taxon-specific parameters}

Comparing results of other primate studies additionally suggests that taxon-specific characteristics are influential. In fact, the optimal home range estimator may even vary for different species living under similar ecological conditions. For instance, MCPs yielded the most accurate estimates for home ranges of northern bearded saki monkeys, whereas the AK method overestimated the area (Boyle et al. 2009). However, the opposite was found for three small, nocturnal species living in rainforest habitats in Central Africa and 
South Asia (central pottos, Perodicticus potto edwardsi and Cross River Allen's galagos, Sciurocheirus cameronensis: Pimley et al. 2005; red slender loris, Loris tardigradus: Kar Gupta 2007). In these studies, kernel estimates were more reliable whereas MCPs tended to overestimate the range sizes. As the habitat characteristics are similar for these species, accuracy of estimates seems to depend rather on taxon-specific traits such as body size, feeding ecology, circadian activity patterns and spatial behaviour (e.g., patrols of territorial boundaries, avoidance of intergroup encounters).

\section{Future implications}

It is obvious from the rapid technological advancement that more and more primate taxa will be equipped with GPS collars in the near future and detailed ranging data of multiple species will become available soon. Therefore, we encourage researchers to take into consideration the following issues when choosing a calculation method and reporting their results:

1. Estimators should correspond to the sample size. Kernel methods seem to be the better choice for small sample sizes (Powell 2000; Burgman and Fox 2003; Grüter et al. 2009; this study) but there are also exceptions (e.g., Boyle et al. 2009). A thorough inspection of the spatial data in ArcView or a similar program helps detecting outliers that could result in overestimation for MCP (e.g., Williams et al. 2008).

2. Appropriateness of an estimator might also depend on the study species'socioecology and the questions that are supposed to be answered. Studies on ecological impact factors, small scale ranging patterns or overall home range size needed to preserve different species demand different solutions. For instance, kernel estimators are probably the better choice to reveal regular seasonal ranging patterns (this study) or for species that concentrate their activity in special areas of the habitat (Row and Blouin-Demers 2006; Boyle et al. 2009). Contrarily, MCP estimates should provide a better image of the total space required by a species, particularly regarding conservation-related questions, or for strongly territorial species that regularly patrol home range borders (Boyle et al. 2009).

3. Results from different estimators (kernel, MCP) and details of the sampling regime (sampling method and sample size over different seasons) show the degree of 
variance in the data and facilitate direct comparisons among different species and study sites (see, e.g., Ostner and Kappeler 2004; Pimley et al. 2005; Harris and Chapman 2007; Irwin 2008; Boyle et al. 2009). If researchers routinely provide these data, effects of different taxon-specific and analytical parameters could be disentangled more easily and, eventually, more precise predictions as well as general conclusions on primate ranging behaviour might be drawn.

\section{Acknowledgments}

We thank the Département de Biologie Animale, Université d'Antananarivo, and the CAFF of the Direction des Eaux et Forêts and the CNFEREF in Morondava for the authorization and support of this study. For assistance in the field we are thankful to Jean-Pierre Tolojanahary, Léonard Razafimanantsoa and the Equipe Kirindy. Franz Kümmeth (e-obs digital telemetry, Grünwald, Germany) provided the GPS-RF collars and excellent technical support. This manuscript benefited greatly from helpful comments by Cornelia Kraus, Melanie Dammhahn, Susanne Schliehe-Diecks and Manfred Eberle. The German Research Foundation (DFG: KA 1082/16-1) kindly provided financial support to LP. 


\section{CHAPTER 3}

CoORdinAtion of GRoup MovementS IN WILD RED-Fronted LEMURS: PROCESSES AND INFLUENCE OF

\section{ECOlogical ANd Reproductive SeAsonality}

with Peter M. Kappeler and Claudia Fichtel

International Journal of Primatology, Special Issue:

Group Coordination \& Decision-Making, accepted with minor revisions (2011) 


\begin{abstract}
Group-living species have to coordinate collective actions to maintain cohesion. In primates, spatial movements represent a meaningful model to study group coordination processes across different socio-ecological contexts. We studied four groups of redfronted lemurs (Eulemur rufifrons) in Kirindy Forest, Madagascar, between 2008 and 2010 across different ecological and reproductive seasons. We collected data on ranging patterns using GPS collars and observational data on different pre-defined parameters of group movements, including initiation, leadership, followership, overtaking events, termination and travel distances. Cohesion of these relatively small, egalitarian lemur groups was high year-round, but daily path length and home range size varied considerably between ecological seasons; presumably due to long distance migrations of some groups at the beginning of the rainy season. Individuals of different age and sex classes successfully initiated group movements. However, stable female leadership prevailed year-round, irrespective of ecological and reproductive season, which might be due to higher or more specific energetic requirements of reproduction. In contrast to lemur species with a more despotic social structure, female red-fronted lemurs did not recruit more followers than males. Adult leaders recruited more followers than subadult ones. Furthermore, recruitment success was higher during the peak of the dry season when predation risk appeared to be higher. Distances of single group movements did neither depend on the initiator's sex and age nor ecological seasons. Our results provide new insights into seasonal variability of coordination processes and the role of social dominance in lemur group movements, thereby contributing to a comparative perspective from a primate radiation that evolved group-living independently of anthropoids.
\end{abstract}

Keywords: leadership; followership; overtaking; cohesion; Eulemur rufifrons

\title{
1 Introduction
}

Living in groups offers a number of individual benefits, including reduced per capita predation risk through shared vigilance or predator confusion (Bertram 1978), as well as opportunities to cooperate with kin, for example in the context of resource defence (Wrangham 1980). However, group-living is also associated with costs because it requires 
consensus decisions in order to coordinate collective actions and to maintain cohesion, both prerequisites for the maintenance of stable social units (Conradt and Roper 2003; King et al. 2008). Throughout the last years, a large body of work on consensus decisionmaking has been published comprising both theoretical (e.g., Conradt and Roper 2003, 2005) and empirical studies (e.g., Kerth et al. 2006; King et al. 2008; Sueur and Petit 2008a, b; Jacobs et al. 2011). The concept of leadership has also attracted much attention (e.g., Rands et al. 2003, 2008; Stueckle and Zinner 2008; King et al. 2009; Lewis et al. 2011). In contrast, only few studies explored in detail the processes underlying coordinated collective actions from initiation to termination (e.g., Trillmich et al. 2004) and their socio-ecological determinants (see also Pyritz et al., in press).

Most group-living species have to move regularly between different foraging patches and sleeping sites. During traveling, groups have to stay cohesive despite interindividual conflicts of interests due to differences in motivation, knowledge or physiological requirements (Conradt and Roper 2005). Therefore, spatial movements represent a salient and meaningful model to explore empirical and theoretical aspects of group coordination (e.g., Petit and Bon 2010; Kappeler 2011). In fact, most empirical studies so far investigated group coordination in the context of collective movements (e.g., Trillmich et al. 2004; Sueur and Petit 2008a, b; Ramseyer 2009; Bousquet et al. 2010; Jacobs et al. 2011). Furthermore, large-scale ranging behaviour and, thus, home range sizes of many species are strongly affected by ecological seasonality (e.g., Doran 1997; Brockman and van Schaik 2004; Scholz and Kappeler 2004), which raises the question whether and how coordination processes of single group movements are also subject to seasonal changes.

Because ranging patterns and inter-individual conflicts among group members vary according to resource availability, predation risk or behavioural seasonality (e.g., Isbell et al. 1990; Doran 1997; Li et al. 2000; Koenig 2002), coordination processes should also depend strongly on these parameters. However, comprehensive studies on group coordination covering different socio-ecological seasons are lacking for primates. For instance, studies on different taxa revealed that rank, sex, age, experience or residence patterns can be defining characteristics of leaders of group-movements (reviewed in King et al. 2009; Fichtel et al. 2011). One possible reason for male leadership is mating competition. For example, male mountain gorillas (Gorilla gorilla berengei) initiate group 
movements after contact with a rival (Watts 1994), and male spider monkeys (Ateles geoffroyi) frequently lead their group to the edge of the home range presumably to make contact with females from other groups (Chapman 1990). On the other hand, female leadership has been attributed to higher energetic needs of females due to gestation and lactation or superior knowledge of resource availability due to female philopatry (Boinski 1988, 1991; Erhart and Overdorff 1999; Trillmich et al. 2004). Because reproduction in many primate species is seasonal (Lindburg 1987), studies should cover reproductive and non-reproductive phases in order to disentangle potential effects of sex, physiological condition, social dominance, residence patterns or experience on leadership (see also Trillmich et al. 2004).

An important first step in this direction is the description of fundamental coordination processes, i.e. which individuals initiate and lead group movements, how many group members follow in which time frame, how far does the group travel and who terminates the movement (e.g., Trillmich et al. 2004). A leader has been defined as an individual that exerts social influence on group members and elicits follower behaviour (King 2010; Petit and Bon 2010; Pyritz et al., in press). In principle, leadership can be distributed over several/all group members or one individual can lead the group consistently (Conradt and Roper 2005; King et al. 2008; Stueckle and Zinner 2008). Leadership in the context of group movements is labelled stable if overtaking during travelling occurs rarely, and as unstable if the leading individual routinely changes within one travel event (Pyritz et al., in press). To fully describe group movements, it is important to study not only which individuals initiate and lead, and how many group members follow because animals face two decisions during group movements, namely (1) when to go and in which direction and (2) where to stop. Thus, it is equally informative to focus on regularities in the termination of group movements (e.g., Trillmich et al. 2004, Pyritz et al., in press).

In the present study, we investigated ranging patterns (home range sizes and daily path lengths) and coordination processes (leadership, overtaking, followership and distances) in movements of four groups of wild red-fronted lemurs (Eulemur rufifrons) throughout a complete annual cycle. Our study species provides a valuable model in this context for at least four reasons having to do with their ecology, reproduction, social structure and evolutionary history. 


\section{Ecology and seasonality}

The study groups are free-ranging and co-reside with a number of different predators (Rasoloarison et al. 1995; Pyritz and Andrianjanahary 2010), which should theoretically provide the necessary level of selective pressure favouring group cohesion, coordination and consensus decision-making (but see Fichtel et al., this issue). The habitat is also strongly seasonal (Sorg and Rohner 1996), and earlier studies revealed pronounced seasonal variation in ranging behaviour according to resource availability, including extraordinary seasonal migrations to places several kilometres outside their usual home range (Kappeler 2000; Scholz \& Kappeler 2004; see also: Overdorff 1993). In addition, redfronted lemurs reproduce seasonally and give birth during the peak of the dry season (Wimmer and Kappeler 2002). Hence, these animals offer the possibility to study effects of ecological variability and related variation in ranging behaviour, as well as reproductive seasonality on coordination processes. We predict that daily path length should be longer during the dry season due to longer movements to scattered resources (e.g., Anderson 1981). We also assume that female leadership should prevail at least during times of gestation and lactation due to increased energetic demands (Erhart and Overdorff 1999; Trillmich et al. 2004).

\section{Social structure}

Most primate species studied so far in coordination research exhibit (relatively) strong dominance hierarchies (e.g., baboons: King et al. 2008; macaques: Sueur and Petit 2008a, b but see Jacobs et al. 2008, 2011), whereas red-fronted lemurs live in small groups characterised by a relatively egalitarian social structure (Kappeler 1991; Pereira and McGlynn 1997). Many previous primate studies showed rank to be a determinant of leadership (white-faced capuchins, Cebus capucinus: Boinski 1993; ringtailed lemurs, Lemur catta: Sauther and Sussman 1993; mountain gorillas: Watts 1994; chacma baboons: Papio ursinus: King et al. 2008), suggesting that social structure has a pervasive impact on coordination processes. Given the lack of clear dominance hierarchies in redfronted lemurs, we expect at least occasionally successful initiations by members of different sex and age classes (see also Jacobs et al. 2008). 


\section{Evolutionary history}

Living in bisexual groups evolved at least twice independently among Malagasy lemurs and only once among the ancestral anthropoids (Kappeler 1999), on which most coordination studies have been conducted to date (e.g., Stewart and Harcourt 1994; Boinski and Campbell 1995; Stueckle and Zinner 2008). Several lemur studies have explored predominantly acoustic coordination mechanisms in solitary and pair-living species that form sleeping groups (golden brown mouse lemurs, Microcebus ravelobensis: Braune et al. 2005; Milne Edwards`sportive lemurs, Lepilemur edwardsi: Rasoloharijaona et al. 2006; red-tailed sportive lemurs, Lepilemur ruficaudatus: Fichtel et al. 2011, this issue). Furthermore, studies on a captive group of brown lemurs (Eulemur fulvus fulvus) provided insights into leadership and decision-making during collective movements (Jacobs et al. 2008, 2011). Finally, two short-term studies have focused on coordination processes during movements of free-ranging groups (Milne-Edwards`sifaka, Propithecus edwardsi and red-fronted lemurs: Erhart and Overdorff 1999; Verreaux's sifakas, Propithecus verreauxi: Trillmich et al. 2004). However, studies exploring coordination in group-living lemurs throughout different socio-ecological seasons are entirely lacking. Therefore, our study also provides comparative data on the convergence of coordination in primate groups in their natural habitat from an evolutionary perspective.

\section{Methods}

\section{Study site and subjects}

We conducted this study at the field station of the German Primate Center (DPZ) at Kirindy Forest, a dry deciduous forest located ca. $60 \mathrm{~km}$ north of Morondava, western Madagascar (Sorg et al. 2003), managed within a forestry concession operated by the Centre National de Formation, d'Etudes et de Recherche en Environnement et Foresterie (CNFEREF). The habitat is characterised by pronounced seasonality with a hot rainy season between November and March and a cooler dry season between April and October (Sorg and Rohner 1996). During the dry season, groups of red-fronted lemurs make daily forays of up to $2 \mathrm{~km}$ to reach ephemeral water holes in the Kirindy river (Scholz and Kappeler 2004; Fig. 1). Reproduction of the species is seasonal, with a 4-week mating season in May-June and a birth season in September-October followed by a 4 month lactation period (Ostner and Kappeler 1999; Barthold et al. 2009). Red-fronted 
lemurs face a number of predators at Kirindy, including the fossa (Cryptoprocta ferox), harrier hawk (Polyboroides radiatus), stray dogs (Canis familiaris) and Malagasy boas (Rasoloarison et al. 1995; Fichtel and Kappeler 2002; Pyritz and Andrianjanahary 2010). The study population inhabits a 60-ha area within the forest featuring a grid system of narrow foot trails with intersections every $25 \mathrm{~m}$ or $50 \mathrm{~m}$, respectively (Fig. 1). Individuals of all study groups have been regularly captured, marked with unique nylon or radio collars and observed since 1996 (Wimmer and Kappeler 2002; Ostner and Kappeler 2004). Infants were marked with nylon collars five to six months after birth and counted as independent group members only from then on (Table 1). Therefore, we were able to identify all study animals rapidly and reliably during observations in the field. Furthermore, individual life histories and (maternal) relatedness were known for most individuals in our focal groups (Table 1).

\section{Data collection}

Four groups of red-fronted lemurs (A: 8-13 individuals; B: 9-12 individuals; F: 9-11 individuals; J: 7-9 individuals) were observed continuously between March 2008 and March 2010 covering all ecological and reproductive seasons (total observation time: $A=484 h ; B=617$ h; F=361 h; J=436 h; all groups=1898 h). For details on group composition and fluctuations within and between groups see Table 1. Observations were conducted between approximately 7:00-10:00h and 14:00-17:00h each day. Each group was observed every second day, alternating between the morning and the afternoon, respectively.

When the group was stationary, we conducted group scans (Altmann 1974) every 15 min (approximately 7600 scans in total) to note (i) the proportion of group members (in \%) within a $10 \mathrm{~m}$ radius around the oldest female of the group and (ii) whether all group members could be detected within a $20 \mathrm{~m}$ radius or not. We randomly selected 80 scans (=approximately $1 \%$ of the total scan number) from different groups for the rainy (November-March) and the dry season (April-October), respectively, for further analyses. Because these results were a prerequisite for the calculation of followership described below, we already present them here. The proportion of group members within a $10 \mathrm{~m}$ radius around the oldest female of the group was slightly higher in the dry $(n=80$; $58.1 \pm 33.5 \%)$ compared to the rainy season $(n=80 ; 57.1 \pm 34.7)$ but the difference was not 
significant (Mann-Whitney $U$ test: $U=3170.5, z=-0.1, p=0.92$ ). There was no difference in the proportion of scans in which we detected all group members within a $20 \mathrm{~m}$ radius between the $\operatorname{dry}(n=80 ; 90.0 \%)$ and the rainy season $(n=80 ; 87.5 \%$; binary logistic regression, code: $1=$ all individuals detected; $0=$ not all individuals detected: $F=0.25$, $p=0.62$ ), either. Given the high degree of group cohesion throughout the year suggested by these results and because red-fronted lemurs constantly produce contact calls while foraging or locomoting (Pereira and Kappeler 1997; Pflüger et al., unpub. data), we assumed individuals of our study groups to be in permanent visual or acoustic contact with each other.

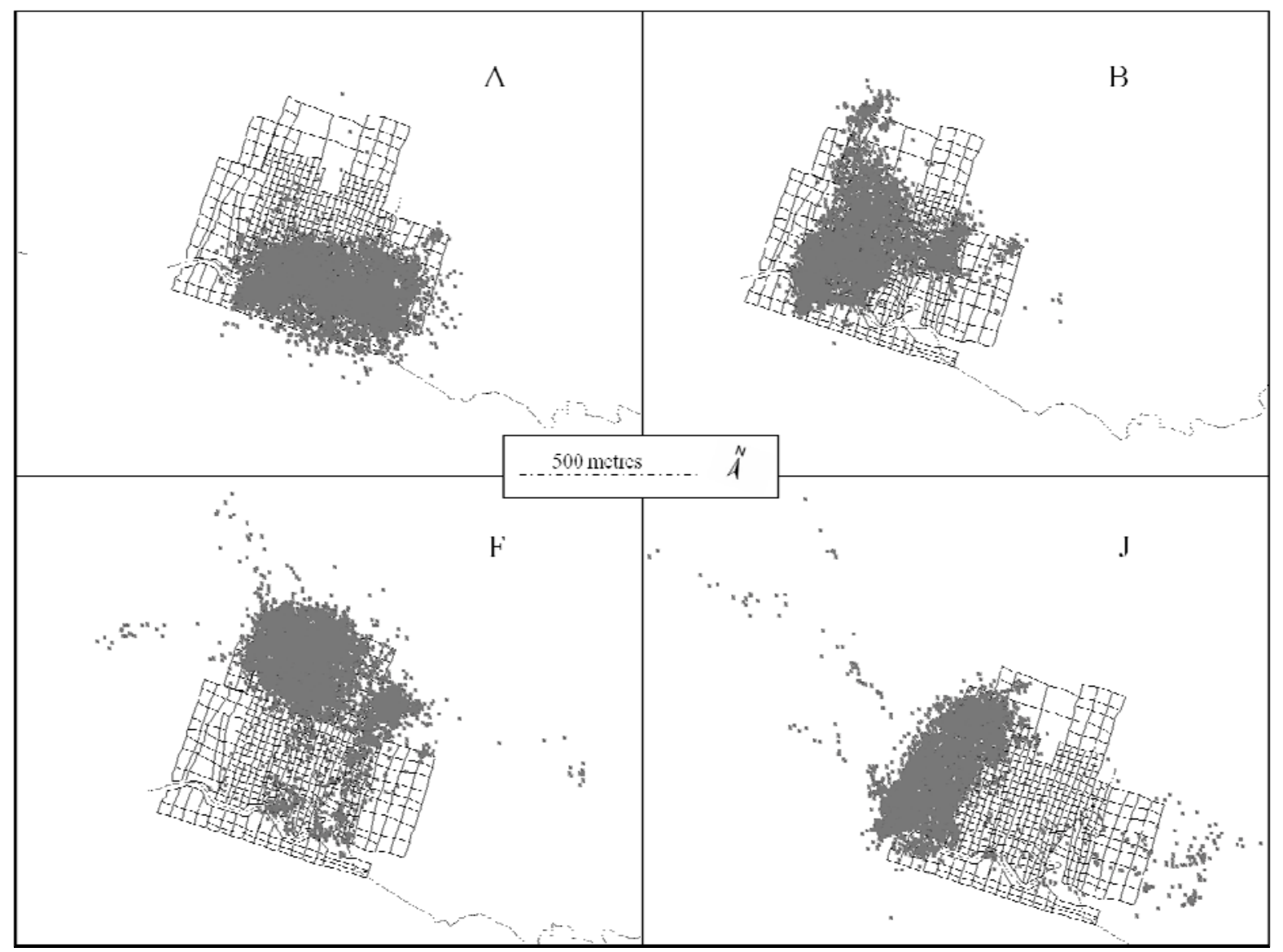

Fig. 1: GPS-fixes of the four study groups (A, B, F, J) collected between March 2008 and September 2009 on a map of the grid system at Kirindy forest. The Kirindy River is depicted by the dotted line to the south of the study site. We present raw GPS data instead of MCP or kernel estimates in order to highlight single excursions of the groups that lead to pronounced differences among different seasons and estimates (Table 1). 
When a group was on the move, we used a previously established operational definition to identify the initiation and termination of a group movement as well as followers and overtaking events. Principally, it has been argued that definitions of group movements should account for a number of taxon-specific characteristics, e.g., different travelling types (directed movements versus 'feed-as-you-go', amoeboid-like movements that do not necessarily require an initiator or coordination among group members, e.g., bonobos, Pan paniscus: Wrangham 2000), mean travel distances, latencies between movements and distances between group members (Pyritz et al., in press). Therefore, we defined group movements based on empirical data from the same groups collected during a pilot study (Pyritz et al. 2010): We observed all group members as focal animals for two hours and recorded any movement of more than three body lengths that occurred during this time, specifying the total distance covered, the spatial distance to the next neighbour after the end of the movement and the latency between two subsequent movements. Based on meaningful breaks in the frequency distributions of these data, a group movement was defined as follows: An individual (= the initiator) that was stationary for at least $4 \mathrm{~min}$ moved at least $15 \mathrm{~m}$ away from its group members in a directed manner without pausing. Other group members were termed followers if their movement diverged no more than 45 degrees from the trajectory of the movement of the initiator and if they arrived in a $6 \mathrm{~m}$-radius around the terminator no more than $10 \mathrm{~min}$ after termination of the movement. That means our definition of followers refers to recruitment success of the initiator measured according to a time and distance threshold. Overtaking occurred if a follower outdistanced the leader, i.e. the individual at the forefront of the group, by more than three body lengths without diverging more than 45 degrees of the initial trajectory of travel. Furthermore, we estimated the distance the group travelled in each movement with the help of the grid cells (Fig. 1) that were additionally subdivided into quarters. A movement was considered as terminated when the leading individual was again stationary for at least $4 \mathrm{~min}$. We did not take into account movements associated with conflict or sexual consort. Because of this definition, we treated all group movements as independent events for statistical purposes.

We used data on all departure attempts to analyse initiatorship, including also movements that we could not follow until termination. All further analyses were conducted using only data of completely observed group movements, including number 
and identity of followers, identity of terminator, potential overtaking events and distances travelled. As in other studies (e.g., Trillmich et al. 2004; Jacobs at al. 2008; Sueur and Petit 2008a), we recorded a successful group movement if the initiator enlisted at least one follower. For calculations on overtaking events and distances of group movements, we did not take unsuccessful attempts (i.e. movements without followers) into account. However, we used them in the analyses of followership because unsuccessful initiations yield information on the social connectivity of initiating individuals (Sueur and Petit 2008a, b). Because the sample size for overtaking events with a subadult initiator was $n=1$, we could not calculate any statistics regarding possible age effects. Since the sizes of our study groups were small and the scans suggested that group cohesion was permanently high, we assumed that each individual had the opportunity to follow each group movement and not only the individuals in a certain radius around the initiator at departure (e.g., Jacobs et al. 2011). Therefore, we measured recruitment success as the proportion of all group members (in \%) following the initiator, corrected for fluctuations of individual group size over time (Table 1).

Behavioural data were collected by LWP (June-August 2008, January-May and September-December 2009); sometimes together with an experienced Malagasy field assistant (March-May and September-December 2008, June-August 2009 and January to March 2010). Group scans were conducted by one observer while groups were stationary. As soon as the group started moving, one observer followed the initiator of a group movement and collected data according to the protocol introduced above. If a second observer was present, he collected data on distances between the initiator and followers and latencies of followers at departure. These data are not included in this paper but are currently analysed for a separate publication on the effects of affiliation and relatedness on follower behaviour. 


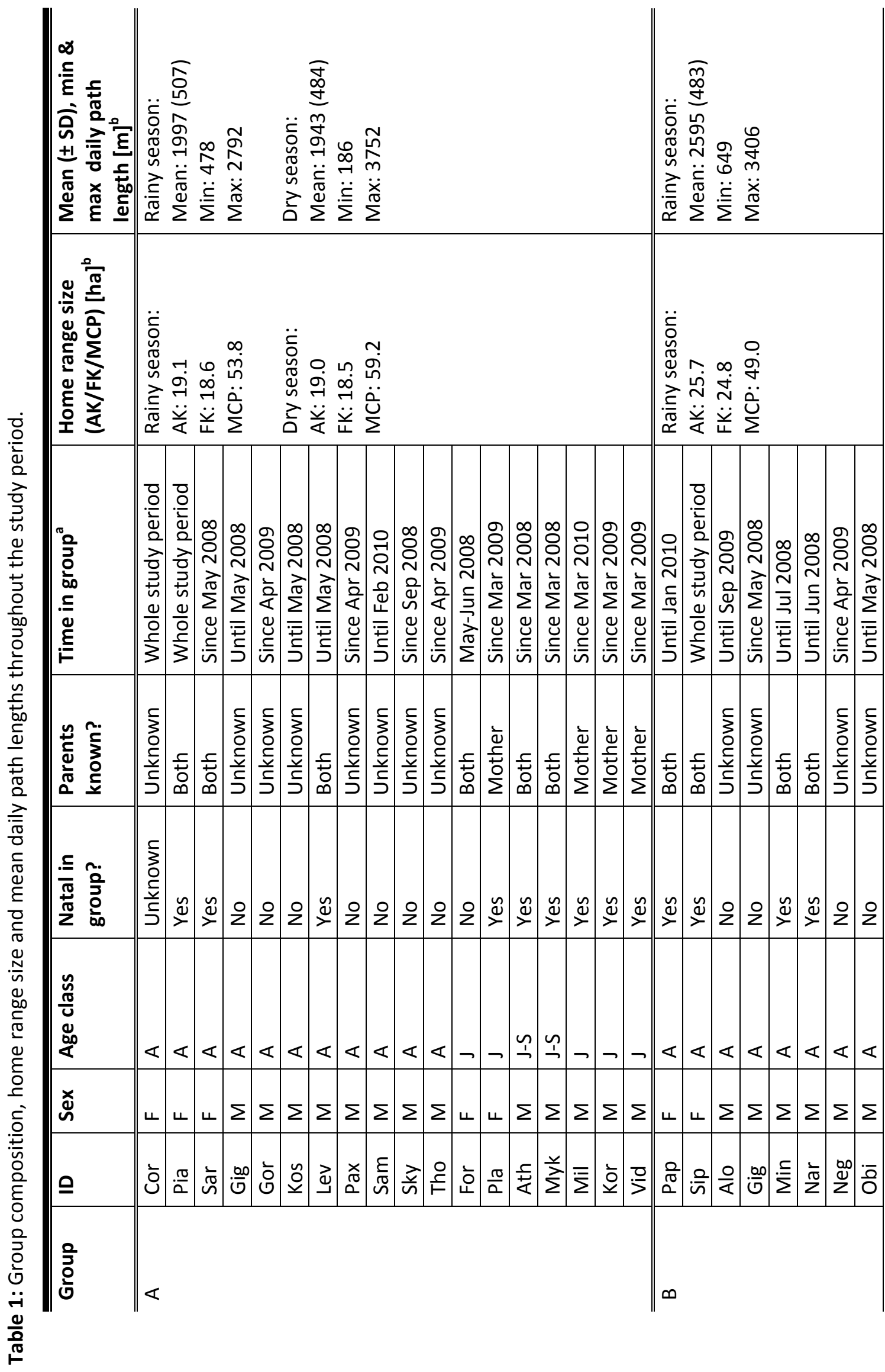




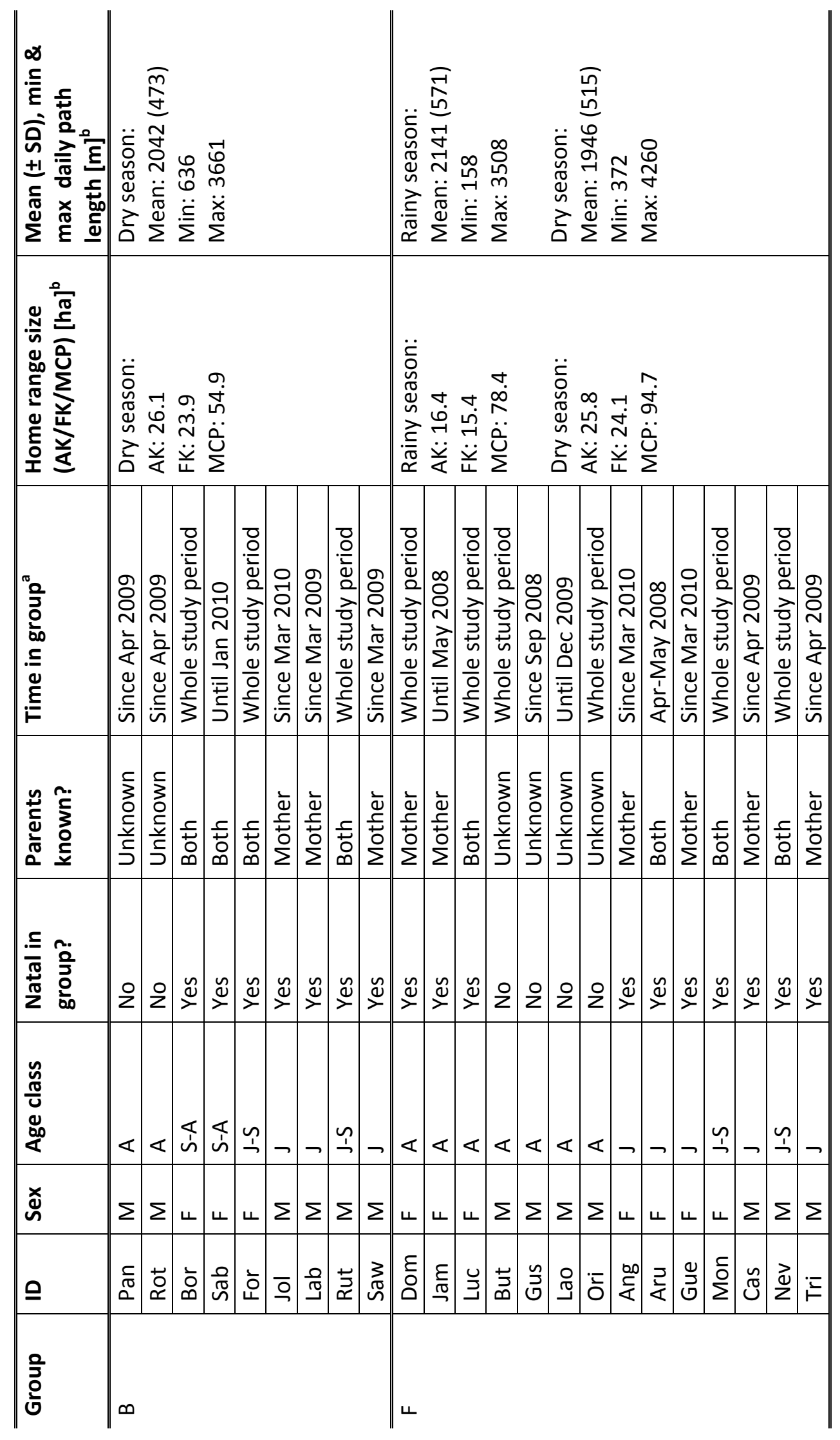




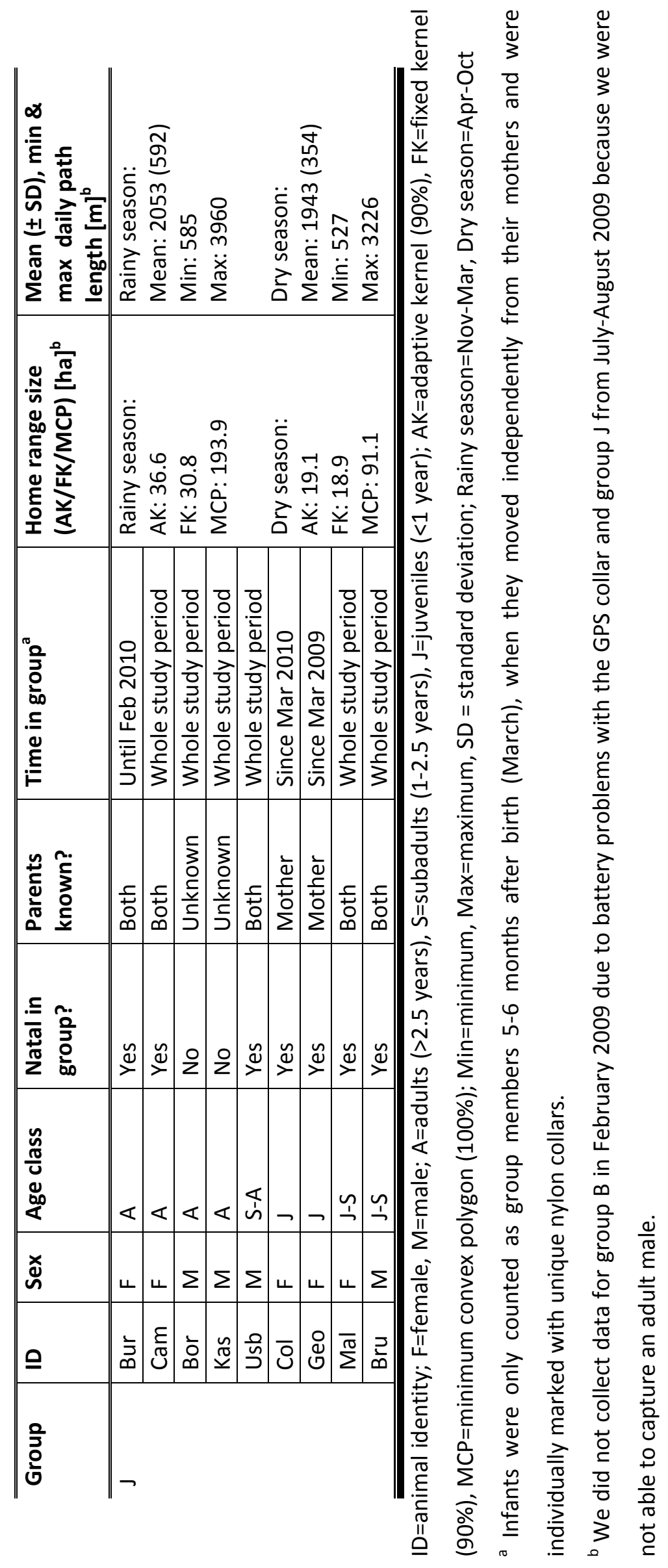


We collected data on ranging behaviour of the groups throughout the study period by fitting one adult male per group with a custom-made GPS-RF tag (for system specification see Kümmeth and Heidrich 2007) from March-June 2008, SeptemberNovember 2008, December 2008-May 2009 and June-September 2009. Because group cohesion was permanently high, we assumed the movements of one group member to provide a realistic proxy of the movement patterns of the entire group. The tags logged spatial position of the animals every 30 min continuously for 2-4 months. Animals were equipped with GPS collars during brief anaesthesia (induced by applying $0.2 \mathrm{ml} \mathrm{GMIl}$; Rensing 1999), following blow-pipe darting by an experienced Malagasy technician. Anaesthetised individuals recovered within two hours and were returned to their social groups. We analysed the 12-months GPS data according to season (rainy: NovemberMarch; dry: April-October). We calculated daily path lengths for each group by adding the distances calculated between subsequent GPS coordinates for each day and seasonal home range estimates with minimum convex polygon (MCP 100\%), as well as with adaptive and fixed kernel (AK 90\%, FK 90\%) methods to provide a measure of data variability (e.g., Boyle et al. 2009; Table 1). We did not exclude any data points because the impact of autocorrelation on home range estimates has been rated as moderate or negligible, especially for large sample sizes (Swihart and Slade 1997; Blundell et al. 2001). Spatial computations were conducted using Home Range Extension (Rodgers \& Carr 2002) for ArcView GIS 3.3 (Environmental System Research Institute [ESRI] 2007).

\section{Statistical analyses}

In order to analyse potential effects of sex, age and seasonality on initiatorship, overtaking events, followership and travelled distances, we calculated a $\chi^{2}$-test, two generalized linear mixed effects models (GLMM) and two linear mixed effects models (LMM; Faraway 2005; Zuur et al. 2009). For details regarding response variables, fixed and random factors of the models see Table 2. Models were fitted using the $R$ software $(R$ Development Core Team, Vienna, Austria, 2010) with the Ime4- and languageR-packages. We used Akaike's Information Criterion (Akaike 1974) to remove parameters stepwise to select the most parsimonious model with the best fit to the data. Factors were excluded only if this 
Table 2: Parameter estimates for the most parsimonious GLMM and LMM. ID=animal identity, $\mathrm{SE}=$ standard error

\begin{tabular}{|c|c|c|c|c|c|c|}
\hline Model & $\begin{array}{l}\text { Response } \\
\text { variable } \\
\end{array}$ & $\begin{array}{l}\text { Random } \\
\text { factors }\end{array}$ & Fixed factors & Estimate & SE & P-value \\
\hline \multirow{5}{*}{$\begin{array}{l}\text { Initiatorship } \\
\text { (binomial } \\
\text { GLMM) }\end{array}$} & \multirow{5}{*}{$\begin{array}{l}\text { Initiation } \\
\text { ("yes"or "no" } \\
\text { for each } \\
\text { individual } \\
\text { in each group } \\
\text { movement) }\end{array}$} & \multirow{5}{*}{$\begin{array}{l}\text { ID nested } \\
\text { in group }\end{array}$} & Intercept & -0.65 & 0.34 & 0.05 \\
\hline & & & $\begin{array}{l}\text { Sex (females > } \\
\text { males) }\end{array}$ & -2.18 & 0.36 & $<0.001 * * *$ \\
\hline & & & $\begin{array}{l}\text { Age (adults > } \\
\text { subadults) }\end{array}$ & -4.42 & 0.39 & $<0.001 * * *$ \\
\hline & & & $\begin{array}{l}\text { Ecological } \\
\text { season }^{a} \text { :sex }\end{array}$ & 0.31 & 0.21 & 0.15 \\
\hline & & & $\begin{array}{l}\text { Reproductive } \\
\text { season }^{\mathrm{b}} \text { :sex }\end{array}$ & -0.29 & 0.50 & 0.69 \\
\hline \multirow{2}{*}{$\begin{array}{l}\text { Overtaking } \\
\text { (binomial } \\
\text { GLMM) }\end{array}$} & \multirow[b]{2}{*}{$\begin{array}{l}\text { Initiator } \\
\text { overtaken } \\
\text { ("yes" or "no" } \\
\text { for each } \\
\text { group } \\
\text { movement) } \\
\end{array}$} & \multirow{2}{*}{$\begin{array}{l}\text { ID nested } \\
\text { in group; } \\
\text { month }\end{array}$} & Intercept & -2.71 & 0.25 & $<0.001 * * *$ \\
\hline & & & $\begin{array}{l}\text { Sex (males > } \\
\text { females) }\end{array}$ & 1.19 & 0.29 & $<0.001 * * *$ \\
\hline \multirow{4}{*}{$\begin{array}{l}\text { Followership } \\
\text { (LMM) }\end{array}$} & \multirow{4}{*}{$\begin{array}{l}\text { Followers } \\
\text { (\% of total } \\
\text { group size) }\end{array}$} & \multirow{4}{*}{$\begin{array}{l}\text { ID nested } \\
\text { in group }\end{array}$} & Intercept & 67.77 & 3.65 & $<0.001^{* * *}$ \\
\hline & & & Sex & -1.51 & 2.85 & 0.60 \\
\hline & & & $\begin{array}{l}\text { Age (adults > } \\
\text { subadults) }\end{array}$ & -21.57 & 9.69 & $<0.05^{*}$ \\
\hline & & & $\begin{array}{l}\text { Season (dry > } \\
\text { other months) }\end{array}$ & -14.82 & 2.75 & $<0.01^{* *}$ \\
\hline \multirow{4}{*}{$\begin{array}{l}\text { Distances } \\
\text { (LMM) }\end{array}$} & \multirow{4}{*}{$\begin{array}{l}\text { Distance } \\
(\mathrm{m})\end{array}$} & \multirow{4}{*}{$\begin{array}{l}\text { ID nested } \\
\text { in group }\end{array}$} & Intercept & 63.50 & 7.63 & $<0.001^{* * *}$ \\
\hline & & & Sex & 8.30 & 5.65 & 0.14 \\
\hline & & & Age & -17.14 & 19.51 & 0.38 \\
\hline & & & Month & 8.32 & 9.80 & 0.52 \\
\hline
\end{tabular}

\footnotetext{
a 2 categories: 1=Nov-Mar (Rainy season); 2=Apr-Oct (Dry season); ${ }^{\mathrm{b}} 4$ categories: 1=May (Mating
} season); 2=Jun-Sep (Gestation); 3=Oct-Feb (Lactation); 4=Mar-Apr (Offspring weaned)

improved the model fit by $>2$ AIC units (Mundry and Nunn 2009). We used maximum likelihood ratio tests to test whether a fixed factor explained a significant amount of the variance and to test the final model with fixed factors against the null model including only the random factors (Faraway 2006). For the LMM, p-values for single fixed factors were not displayed in the model summary. We used Markov chain Monte Carlo methods to generate $p$-values for these parameters instead (Bates and Sarkar 2007). We used a Mann-Whitney $U$ (MWU) test to compare daily travel paths between different ecological seasons. The significance level was set at $\alpha=0.05$ for all tests. In order to be able to graphically present the results of the binomial GLMM on initiations, we calculated mean individual initiation rates per hour for each month (Fig. 2). 


\section{Results}

\section{General ranging patterns}

GPS-tracking revealed that daily path length of a group ranged from $158 \mathrm{~m}$ to $4260 \mathrm{~m}$ $(n=1107)$ with an overall mean ( \pm standard deviation) of $2047( \pm 129)$ m. Mean daily path length during the rainy season $(2196 \pm 272 \mathrm{~m} ; \mathrm{n}=238)$ was significantly longer than during the dry season (1968 $\pm 49 \mathrm{~m} ; \mathrm{n}=869$; MWU test: $U=78891.0, z=-5.5, p<0.001$ ). Home range size did not vary between ecological seasons for group A and B but did vary considerably between ecological seasons for groups $\mathrm{F}$ and $\mathrm{J}$, and numerical estimates varied widely depending on the particular estimator used (Table 1). While kernel estimates (AK and FK combined) ranged from a minimum of 15.4 ha to a maximum of 36.6 ha, with a mean of $22.3 \pm 4.8$ ha, MCP estimates ranged between 49.0 ha and 193.9 ha with a mean of $84.4 \pm 47.6$ ha.

\section{Initiatorship}

We observed a total of 721 initiation attempts by adults (females=564; males=139) and subadults (females=6; males=12). Females did initiate group movements most often throughout the year, irrespective of reproductive or ecological season (Fig. 2; Table 2). The final binomial GLMM with single initiation events as depended variable ("yes" for the initiator "no" for every other group member for each group movement to control for sexand aged-biased group composition and fluctuations over time), sex and age as fixed factors and animal identity nested in group as random factor performed significantly better than the null model $\left(n=721 ; G L M M: \chi^{2}=163.9, d f=2, p<0.001\right)$. Further analyses were conducted using only data of completely observed group movements $(A=202$; $B=251 ; F=106 ; J=141$; total=700), of which 660 (94.3\%) were successful, i.e. enlisted at least one follower. There was no difference concerning sex of unsuccessful initiators (females=21; males=19; $\chi^{2}$-test: $\chi^{2}=0, d f=1, p=1$ ) and unsuccessful attempts occurred year-round in both sexes without seasonal patterns. 

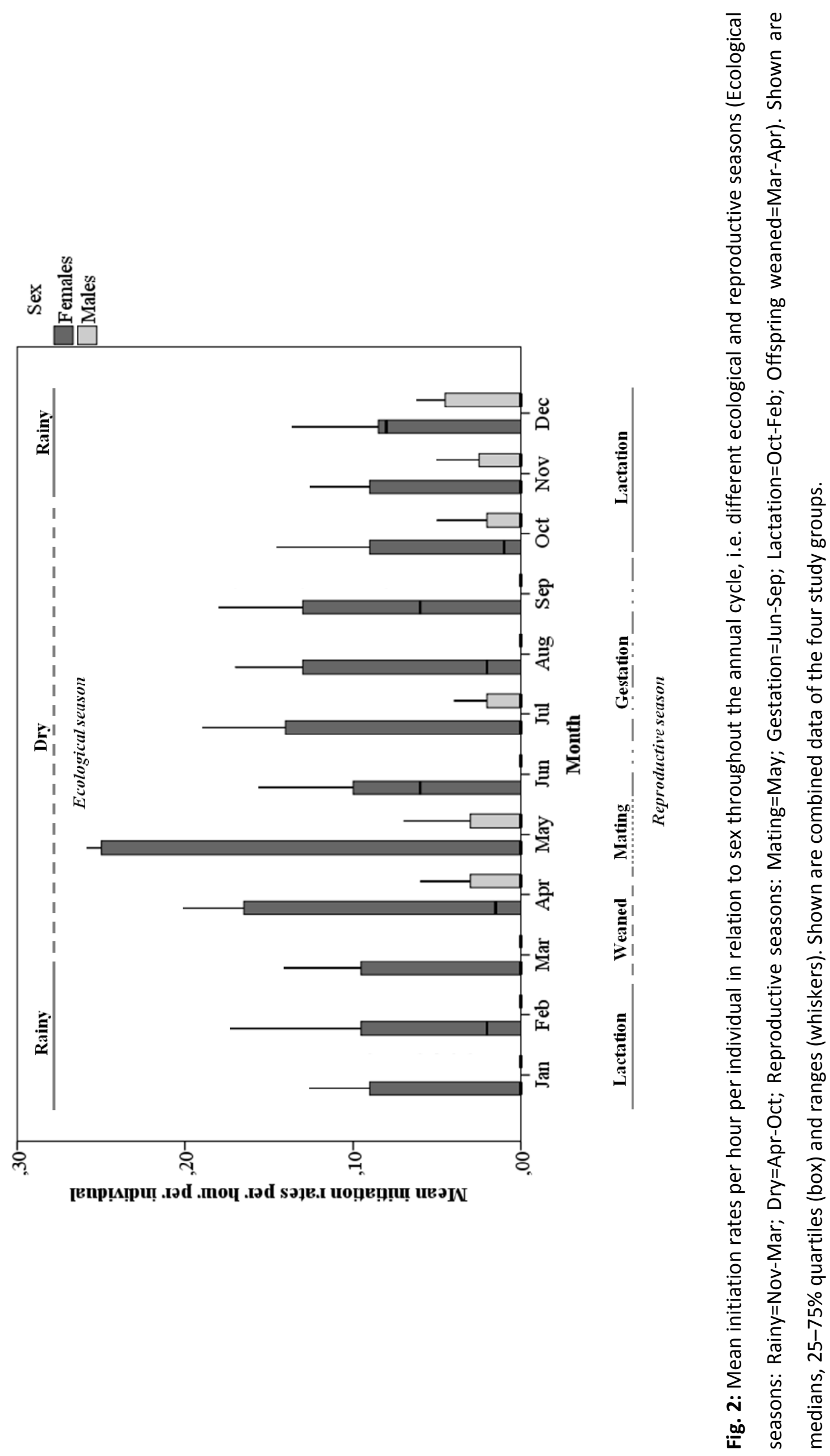


\section{Overtaking}

In general, overtaking events were relatively rare $(n=62)$; i.e., the occurred during only $9.4 \%$ of 660 successful movements. Hence, the initiator of a group movement was also the terminator in the vast majority of all movements. The model including sex as fixed factor and month and individual identity nested within group as random factors explained significantly more variance regarding overtaking events than the null model $(n=660$; GLMM: $\left.\chi^{2}=10.8, d f=1, p<0.001\right)$ : Male initiators were overtaken more often than female initiators (females: 7.0\%; males: 20.2\%; Fig. 3; Table 2). Overtaking occurred without aggression in $100 \%$ of observed cases.

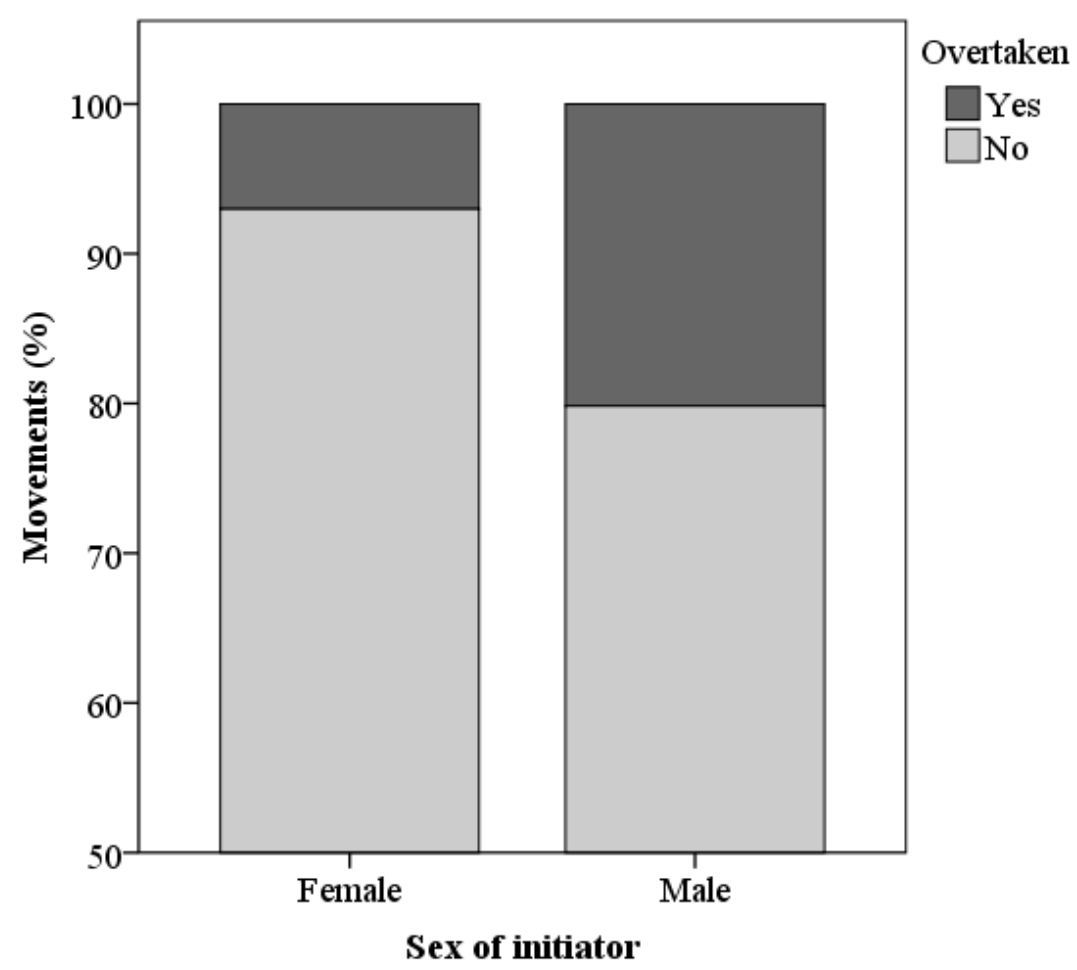

Fig. 3: Proportion of overtaking events in relation to sex of the initiator. Shown are percents of group movements. $N_{\text {movements (total/overtaken) }}=541 / 38$ for females; $N_{\text {movements (total/overtaken) }}=119 / 24$ for males. Note that the ordinate starts at $50 \%$. Shown are combined data of the four study groups.

\section{Followership}

On average, adults were followed by a higher percentage of group members $(n=691$; $55.8 \pm 29.8 \%)$ than subadults $(n=9 ; 31.7 \pm 21.5 \%)$ when leading a group movement (Fig. 4). Furthermore, initiators had more followers during the peak dry season between September and November $(n=207 ; 67.0 \pm 31.7 \%)$ compared to other months/seasons 
( $n=493 ; 50.7 \pm 27.7 \%$; Fig. 5). In contrast, sex had no effect on the number of recruited group members (females: $n=562 ; 55.6 \pm 28.8 \%$ followers; males: $n=138 ; 55.1 \pm 33.7 \%$ followers). The respective model performed significantly better than the null model ( $n=700$ LMM: $\chi^{2}=44.9, d f=3, p<0.001 ;$ Table 2).

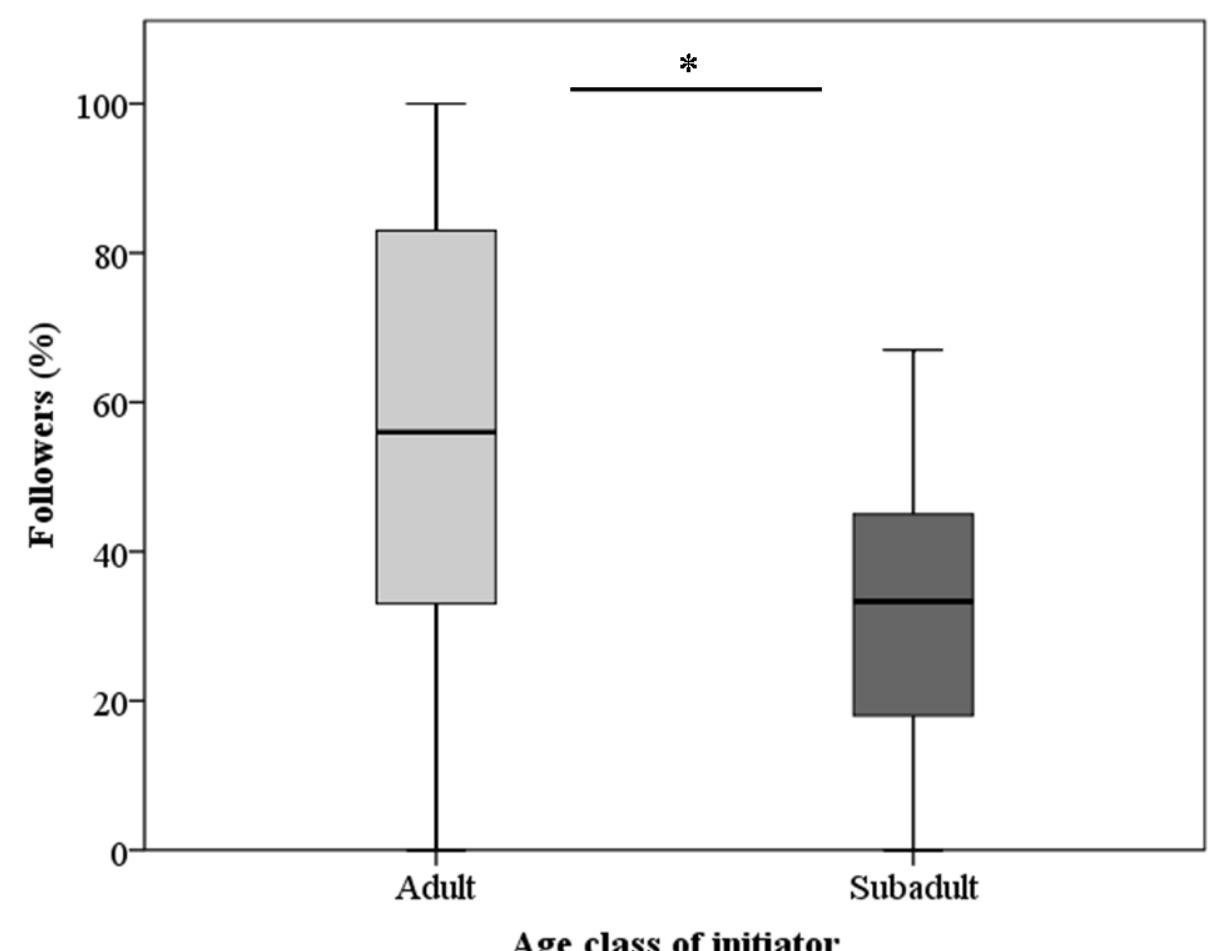

Fig. 4: Percent followers during group movements in relation to age class of the initiator. Shown are medians, $25-75 \%$ quartiles (box) and ranges (whiskers). $\mathrm{N}=691$ for adults; $\mathrm{N}=9$ for subadults. * indicates $p<0.05$. Shown are combined data of the four study groups.

\section{Distances of single group movements}

Distances of single group movements ranged from $15 \mathrm{~m}$ to $580 \mathrm{~m}$, with a median of 60.0 $\mathrm{m}$. Neither sex nor age of the initiator affected distances covered in a single movement, i.e. group movements that were led by a female $(n=562 ; 71.2 \pm 53.1 \mathrm{~m})$ or adult $(n=691$; $72.8 \pm 54.5 \mathrm{~m}$ ) did not cover significantly larger distances than those led by males $(n=138$; $77.8 \pm 58.8 \mathrm{~m}$ ) or subadults $(\mathrm{n}=9 ; 55.0 \pm 28.5 \mathrm{~m})$. Furthermore, distances of observed group movements did not vary significantly among months. Accordingly, the respective model did not perform better than the null model ( $n=660$ LMM: $\chi^{2}=15.0, d f=13, p=0.31$; Table 2). 


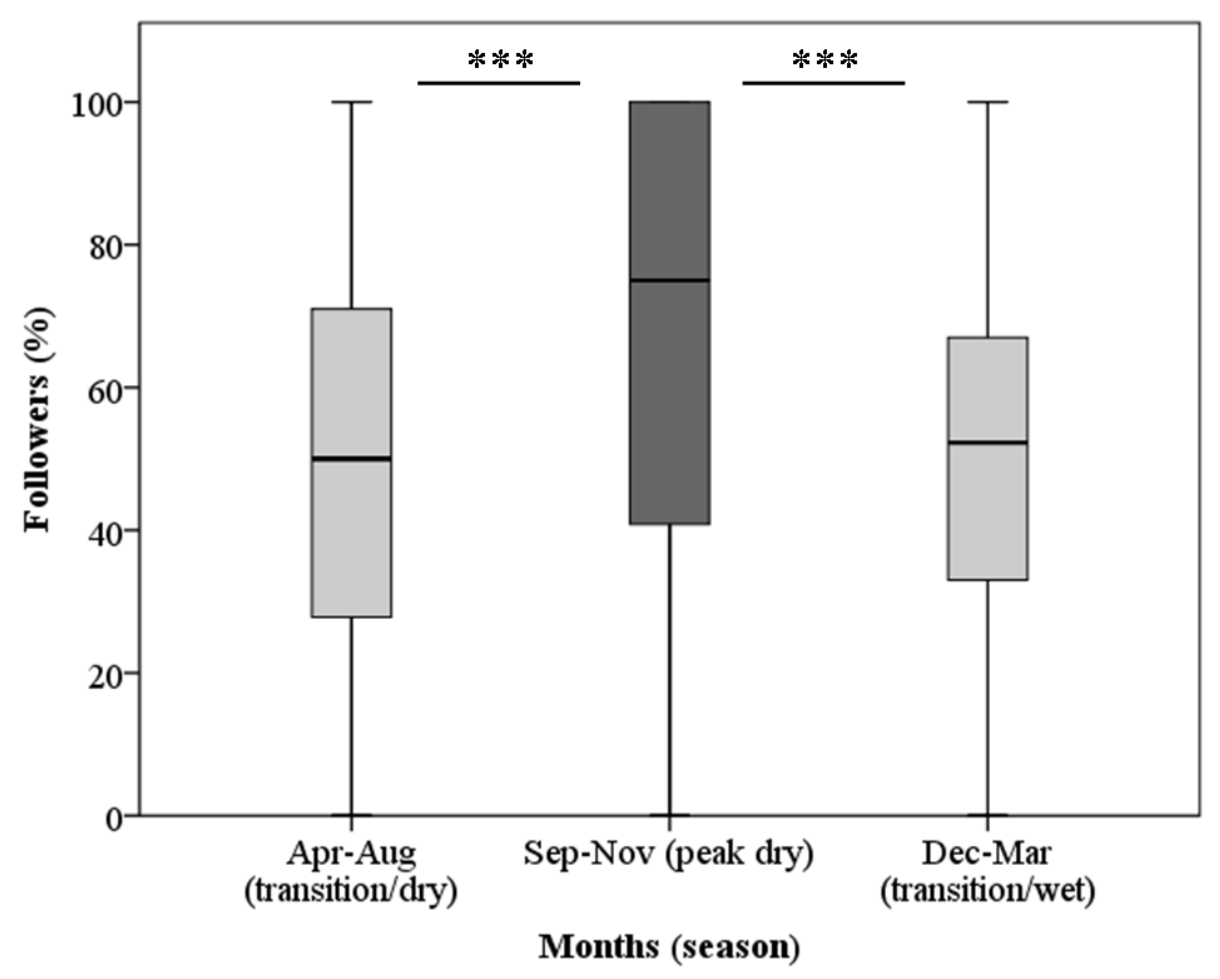

Fig. 5: Percent followers during group movements in relation to ecological season. Shown are medians, 25-75\% quartiles (box) and ranges (whiskers). N=299 for (transition to) dry season (April-August); $\mathrm{N}=207$ for peak dry season (September-November); $\mathrm{N}=194$ for (transition to) rainy season (December-March). ${ }^{* * *}$ indicates $p<0.001$. Shown are combined data of the four study groups.

\section{Discussion}

We analysed ranging patterns and processes of group movements in free-ranging redfronted lemurs throughout different seasons. Mean daily path length was longer during the rainy, compared to the dry season. Home range size estimates varied strongly in two groups depending on season $(F, J)$ and estimator (all groups). Females and males of different age classes successfully initiated group movements but adult females did so most often, irrespective of ecological and reproductive season. However, female initiators did not enlist more followers than males. Adult individuals had higher recruitment success. Furthermore, recruitment success was higher at the peak of the dry season compared to other months. Neither sex nor age of the initiator nor season did affect distances of single movements. We discuss each of these findings in more detail below. 


\subsection{General ranging patterns}

Home range use throughout different seasons

Home range size did not differ across seasons in group $A$ and $B$, which might be due to the fact that their home ranges are close to the river, so that daily excursions to water holes as in group $\mathrm{F}$ were not necessary. In contrast, home range estimates differed considerably with season, especially in groups $F$ and J (Table 1). In group F, this difference might be due to daily excursions to water holes during the dry season because their home range is furthest away from the Kirindy river (see also Scholz and Kappeler 2004). Furthermore, group $\mathrm{F}$ conducted migrations out of the regular home range on February 7 and March 17, 2009. Group J conducted a similar migration to a far-off area between March 16 and 28, 2009 (see also Kappeler 2000). Unfortunately, we were not able to observe these migrations directly. However, subsequent surveys suggested that they lead to patches of preferred feeding trees of the lemurs with short flowering or fruiting intervals between January and March, including Astrocassine pleurostyloides, Strychnos decussata and Berchemia discolour (see Sorg and Rohner 1996).

Similar observations of migrations to remote resources were reported from redfronted lemurs in Ranomafana, which left their usual home range during a time of low fruit availability to feed in a plantation of Chinese guava (Psidium cattleyanum) (Overdorff 1993). Pronounced seasonal home range changes based on detailed ranging data have been described for many anthropoid species as well (e.g., red colobus, Colobus badius tephrosceles: Clutton-Brock 1975; chacma baboons: Anderson 1981; Sichuan snub-nosed monkey, Rhinopithecus roxellana: Li et al. 2000; white-faced capuchin monkeys: Baker 2001). However, none of these studies has looked at the effects of seasonality on coordination processes at the same time so that a comparative discussion of our findings in this context is currently not possible. 


\section{Daily path length}

In contrast to our expectation, mean daily path length was larger in the rainy, compared to the dry season in all groups (Table 1). This could be due to longer daily travel paths of groups $\mathrm{F}$ and $\mathrm{J}$ during their migrations in February and March (mean daily path length during these migrations: $2464 \pm 746 \mathrm{~m}$ ). Contrarily, daily path length has been reported to increase during the dry season due to longer foraging movements in chacma baboons (Anderson 1981), vervet monkeys (Cercopithecus aethiops: Struhsaker 1967b) and Verreaux`s sifakas (Richard 1978). Thus, it may be that red-fronted lemurs at Kirindy respond to ecological constraints by reducing activity in order to save energy.

\subsection{Coordination of group movements}

Initiation, overtaking and termination

According to our prediction, group members of all sex and age classes initiated group movements across the year. However, adult females did so most often, irrespective of ecological and reproductive season and related seasonal variation in home range use. Overtaking occurred rarely, and female initiators were less often overtaken than male initiators. Hence, females that initiated a group movement also terminated it in almost all cases, suggesting that females do have a strong influence on other group members regarding decisions on when and where to move and where to stop. Interestingly, if overtaking events occurred, they were never accompanied by aggressive behaviour. This could mean that decisions about where to go do not create conflicts that need to be resolved aggressively. However, termination of a group movement might already be predetermined by a goal-directed departure (Sueur et al. 2010) or be based on a second consensus decision, so that overtaking events do not necessarily imply that the decision where to stop will be made by the overtaking individual (Pyritz et al., in press). In fact, aggressive behaviour during decision making processes has so far not been reported in any species, indicating that decisions about when and where to go might be based on consensus decisions at departure or destination (see also Conradt \& Roper 2007). 
Leadership in free-ranging and captive groups

In accordance with our prediction, red-fronted lemurs displayed distributed, but highly skewed and stable leadership of adult females, which corresponds to the results of a previous study on red-fronted lemurs in a rainforest habitat (Erhart and Overdorff 1999). Interestingly, however, a study on a captive group of brown lemurs did not reveal any effect of sex or age on coordination of group movements (Jacobs et al. 2008). Because, this group was housed in a 0.2 ha enclosure and did neither experience predation risk nor seasonal (resource) variation, these conditions may promote less skewed and equally shared leadership. Hence, comparative studies in captivity and in the wild can reveal important insights about factors explaining why certain individuals exert more social influence.

\section{Female leadership and physiological demands of reproduction}

Female leadership has been discussed as a non-aggressive strategy to enhance individual foraging efficiency and nutritional intake in order to compensate added energetic costs of gestation and lactation in a number of anthropoid and lemur species (squirrel monkeys, Saimiri oerstedi: Boinski 1988, 1991; diademed sifakas, Propithecus diadema edwardsi and red-fronted lemurs: Erhart and Overdorff 1999; Verreaux`s sifakas: Trillmich et al. 2004). However, reproduction in red-fronted lemurs is seasonal, i.e. mating, gestation and lactation take place between May and February (Barthold et al. 2009). Nevertheless, female leadership occurred year-round without significant differences among months.

Energetic costs of reproduction accrue primarily through lactation and infant carrying (Pond 1977; Oftedal 1985; Tarnaud 2006). Thus, females may continue initiating and leading group movements between the weaning of an infant and the next mating season (February-May) in order to compensate for the physiological depletion of the past months and/or to store fat in advance of the next gestation period, as suggested for ringtailed lemurs (Pereira 1993). However, lactating brown lemurs increased their food intake only during the early lactation period (first 3 months) when the frequency of suckling is highest and infants do not yet consume solid food items independently (Tarnaud 2006). Thus, quantitative energetic demands seem to decrease over the course of the Eulemur lactation period, making compensatory post-weaning leadership of females less likely. 
However, other studies did reveal a strong impact of nutritional provisioning on reproduction in lemurs, i.e. females tended to give birth to twins instead of one single infant when resources were plentiful (ringtailed lemurs: Nunn and Pereira 2000). Thus, detailed data on nutritional intake and quality of consumed food items are required for a better understanding of female energetic demands. For instance, females may lead the group to resources providing them with specific micro-nutrients they lack after lactation during the migrations at the beginning of the rainy season, which coincide with the end of the weaning process. Future studies exploring this sex difference in more detail would ideally also include analyses of sex differences in metabolic rate (e.g., Simmen et al. 2010) or motivation to exploit specific resources (see also King et al. 2009).

\section{Female leadership and philopatry}

An alternative explanation for year-round female leadership might be the fact that females are the predominantly philopatric sex in red-fronted lemurs. Philopatry may involve better knowledge of the home range and more experience regarding (seasonal) resource distribution (e.g., long-tailed macaques, Macaca fascicularis: van Noordwijk \& van Schaik 1987; gorillas: Watts 1994). This could especially account for the extraordinary movements during the rainy season to scattered, ephemeral resources mentioned above. However, females are also the philopatric sex in chacma baboons, but males initiate and lead more group movements in this species - although the sex-specific difference regarding initiatorship is not as pronounced as in red-fronted lemurs (Stueckle and Zinner 2008). Therefore, characteristics of the social structure, e.g., male dominance in chacma baboons (Bulger 1993), might have a greater effect on coordination and leadership than residence patterns, but, clearly, more studies on species with additional combinations of residence and dominance patterns are required to disentangle these factors.

The findings regarding the distances of single group movements do also not support the philopatry hypothesis as a reason for female leadership: Sex and age class of the initiator did not influence distances of single group movements and month did not have an effect, either. Additionally, the mean distance of movements was short (ca. 70 $\mathrm{m})$. Hence, there seems to be a rather small-scale distribution pattern of feeding and resting sites in the home range throughout the year which does not require long 
movements between scattered resources by particularly well informed individuals, even not for the extraordinary migrations of groups during the rainy season.

\section{Social structure, recruitment success and expected decision-type}

Philopatric female red-fronted lemurs are usually closely related to several other members of their group, e.g., sisters or their offspring from previous years, and are well connected in affiliative social networks, whereas males switch groups more often (Wimmer and Kappeler 2002; Ostner and Kappeler 2004). It has been proposed that group members with strong socio-genetic network relations will more often and/or more rapidly be followed when initiating a movement, and thus be more likely to act as leaders (King et al. 2009; King and Sueur, in press). In fact, King et al. (2008) found that close social ties to the male leader mediated consensus costs of follower behaviour in chacma baboons travelling to experimental food patches. Furthermore, Jacobs et al. (2011) showed that affiliative network relations among individuals were the best predictor regarding joining of group movements in a captive group of brown lemurs, i.e. an individual's decision to join a movement depended on the decision taken by its preferred social partners.

However, female red-fronted lemurs were not more successful in recruiting followers than males. Hence, network relations do not seem to be the only determinant of recruitment success. On the other hand, in Verreaux`s sifakas which also display female philopatry (Brockman 1999) and predominantly female leadership, female initiators had a higher recruitment success than males (same operational definition of followership as in this study: Trillmich et al. 2004). This contrast might be related to different dominance patterns: Adult females are socially dominant over males in sifakas (Richard 1987) but not in red-fronted lemurs (Pereira et al. 1990). Hence, social dominance of females seems to have a strong influence on the joining behaviour of conspecifics. The lack of social hierarchy in our study species could also explain the relatively high proportion of successfully led group movements by males (see also Erhart and Overdorff 1999) compared to other primate species with pronounced female dominance (squirrel monkeys: Boinski 1991; ringtailed lemurs: Sauther and Sussman 1993; diademed sifakas: Erhart and Overdorff 1999; but see Verreaux`s sifakas: Trillmich et al. 2004). 
The joining behaviour of group members, i.e. the decision-making process on the individual level, can also be linked to social relationships among individuals. For instance, an egalitarian social structure characterised by tolerant relations among group members coincided with equally shared decision-making involving a voting process (quorum) in Tonkean macaques (Macaca tonkeana) whereas rhesus macaques (Macaca mulatta) with their more pronounced dominance hierarchy exhibited only partially shared consensus decisions (Sueur and Petit 2008a, b; Sueur et al. 2010). Furthermore, Jacobs et al. (2011) showed that joining behaviour in brown lemurs depended on previous actions of the preferred social partner, i.e. the decision was not made by a single leader but rather selforganized with each individual being able to influence the decision outcome. Given the egalitarian social structure of our study species, we would also expect shared consensus decision-making in red-fronted lemurs during departure, i.e. the actions of several group members should contribute to the decision outcome.

\section{Age, ecological seasonality and recruitment success}

In many primates, adult and, thus, supposedly more knowledgeable and experienced group members initiate and lead group movements more often than subadults (Japanese macaques, Macaca fuscata: Itani 1963; chimpanzees, Pan troglodytes: Boesch 1991a; squirrel monkeys: Boinski 1991; gorillas: Stewart and Harcourt 1994; white-faced capuchin monkeys: Boinski and Campell 1995). We also observed mostly adult individuals initiating and leading group movements in red-fronted lemurs.

Furthermore, recruitment success seemed to be season-dependent because the number of followers during the peak dry season was significantly higher than in the months before and after. This variability of group cohesion during movements between different seasons might be due to a number of reasons: Low resource abundance during the peak dry season may have resulted in more targeted movements of the whole group to single feeding trees and water holes compared to other months (e.g., Scholz and Kappeler 2004). Second, increased predation risk in the dry season should promote group cohesion, for instance, fossas prey more on lemurs during the dry season at Kirindy when their main rainy season prey (tenrecs) are hibernating (Hawkins and Racey 2008). In fact, predation risk is supposed to be generally higher when the habitat is more open due to defoliated trees (e.g., Janson and Goldsmith 1995). Finally, group cohesion might be 
higher due to increased between-group competition about scarce resources during the dry season (Cheney 1992).

However, why is the suggested difference in group cohesion not reflected in the scan data presented in the method section? Perhaps the groups were only more cohesive during travelling but not during stationary behaviour. Furthermore, the scan data are not directly comparable to the recruitment data because followers could arrive within close proximity to the terminator in a $10 \mathrm{~min}$ time window and did not have to stay there for a certain period. Finally, the resolution of the scan data $(\leq 10 \mathrm{~m}) \mathrm{might}$ be too low to detect seasonal differences in cohesion. Ideally, operational definitions of followership in future studies should take seasonal variation of group cohesion into account by assessing season-specific thresholds, e.g., individuals have to arrive within a $6 \mathrm{~m}$ radius around the terminator during the dry season but only within a $9 \mathrm{~m}$ radius in the rainy season to be termed followers (see also Pyritz et al. in press).

\section{Conclusions}

Our results indicate variation in follower behaviour in a free-ranging lemur species according to the age of the initiator and the ecological context. However, not all features varied with environmental conditions: Female leadership - that has been described for many anthropoid and lemur species - prevailed year-round, perhaps due to special nutritional needs related to gestation and lactation. A comparison among other (lemur) taxa suggested that social dominance patterns seem to influence follower behaviour, i.e. this egalitarian species did not display sex-biased recruitment success in contrast to despotic anthropoid and lemur taxa. Our results provide a comprehensive example of group coordination processes in lemurs that evolved group-living independently of anthropoids. In the future, more studies on different primate taxa can build on these data to disentangle the effects of sex, age, dominance relations and residence patterns and illuminate the evolutionary roots of coordination, leadership and decision-making in freeranging primates.

\section{Acknowledgements}

We acknowledge the authorization and support of this study by the Département de Biologie Animale, Université d'Antananarivo, the CAFF of the Direction des Eaux et Forêts 
and the CNFEREF Morondava. For support and assistance in the field we are deeply thankful to Jean-Pierre Tolojanahary, Léonard Razafimanantsoa, Tianasoa Andrianjanahary and the Equipe Kirindy. This manuscript benefited greatly from discussions with Dagmar Clough, Elise Huchard, Andrew King, Cornelia Kraus and Cédric Sueur and comments by two anonymous reviewers. The German Research Foundation (DFG: KA 1082/16-1) and the German Academic Exchange Service (DAAD) kindly provided financial support. We are also grateful to Joanna Setchell for her support during preparing this special issue, and the International Journal of Primatology for funds that contributed towards the travel costs to the XXIII Congress of the International Primatological Society (IPS) in Japan. 


\title{
CHAPTER 4
}

\author{
DeterminANTS AND OUtCOMES OF DeCISION-MAKING, \\ GROUP COORDINATION AND SOCIAL INTERACTIONS DURING A \\ FORAGING EXPERIMENT IN A WILD PRIMATE
} with Claudia Fichtel, Elise Huchard and Peter M. Kappeler 


\begin{abstract}
Social animals have to coordinate joint movements to maintain group cohesion, which is often compromised by diverging individual interests. A widespread behavioural mechanism to achieve coordination is through consensus decision-making that can be either shared or unshared. If consensus costs are high, group fission represents an alternative tactic. Exploring determinants and outcomes of spontaneous group decisions and coordination of free-ranging animals is methodologically challenging. We therefore conducted a foraging experiment with a group of wild red-fronted lemurs (Eulemur rufifrons) to study decision outcomes, coordination of movements, individual foraging benefits and social interactions in response to the presentation of drinking platforms with varying baiting patterns. Behavioral observations were complemented with data from recordings of motion detector cameras installed at the platforms. Observations during the experimental conditions were compared to findings from natural group movements. Unfortunately, we could not determine the type of consensus decision-making because the group visited platforms randomly. As under natural conditions, adult females initiated most group movements, but overtaking by individuals of different age and sex classes occurred in $67 \%$ of movements to platforms, compared to only $18 \%$ during other movements. As a result, individual resource intake did not depend on departure position, age or sex, but on arrival order. The group fissioned during $10.9 \%$ of platform visits and fissioning resulted in more individuals drinking simultaneously. Aggressiveness at the platforms did not affect resource intake, presumably due to low supplanting rates. Our findings highlight the variability of coordination processes and related consequences for individual foraging benefits in a wild primate group.
\end{abstract}

Keywords: consensus decision; group fission; cohesion; drinking platforms; red-fronted lemurs

\title{
1 Introduction
}

Group-living holds a number of benefits, but also costs for individual group members. Many of the benefits such as shared vigilance or predator confusion are related to reduced per capita predation risk, whereas costs of group-living include increased intragroup feeding competition (Bertram 1978; Krause and Ruxton 2002; Fichtel in press). 
Permanent cohesion is a prerequisite to reap the benefits associated with group-living, and consensus decisions enable group members to coordinate their activities and travel schedules (Conradt and Roper 2005; Conradt and List 2009). However, joint decisionmaking underlying cohesion can be hampered by diverging individual needs due to differences in sex, age, motivation as well as reproductive and physiological state among group members (Rands et al. 2008). Two principal ways have been identified with which group-living animals can reach a consensus decision despite these inter-individual conflicts. In (partially) shared consensus decisions, each individual (or at least a subset of group members) has the opportunity to influence the outcome of a decision in a voting process. In contrast, in unshared consensus decisions, only one individual decides when to change place and activity, irrespective of other individuals' interests, and all group members abide by this decision (Conradt and Roper 2005).

Group fission, i.e. temporary splitting of a group into two or more subgroups, has often been interpreted as the outcome of a failed consensus decision due to incompatible individual interests (Franks et al. 2003; Conradt and Roper 2005). However, fissioning may also allow group members to avoid costly consensus decisions under certain conditions without foregoing the benefits of group-living for a long time (Kerth 2010). For instance, fissioning has been observed in recent studies on group coordination and decision-making in homing groups of domestic pigeons (Columba domestica: Biro et al. 2006) and in Bechstein's bats (Myotis bechsteinii) switching communal roosts (Kerth et al. 2006). In a recent field experiment, temporary splitting into subgroups has also been observed in a foraging troop of chacma baboons (Papio ursinus) visiting small artificial food patches when followers had weak social ties with the dominant male leading the group (King et al. 2008).

Most empirical and theoretical studies explore decision-making and coordination in the context of group movements because they provide a biologically meaningful model and uniform context to study determinants and consequences of relevant coordination processes (e.g., modelling: Rands et al. 2008; rhesus and Tonkean macaques, Macaca mulatta and M. tonkeana: Sueur and Petit 2008a, b; domestic geese, Anser domesticus and heifers, Bos taurus: Ramseyer 2009a,c; meerkats, Suricata suricatta: Bousquet et al. 2010). In order to operationalise and quantify the coordination of group movements, it has proven useful to divide them into successive phases that are defined taxon- 
specifically to determine which individuals initiate, whether the leader is overtaken, who terminates the movement, how many group members follow in which time frame, and how far the group travels (Trillmich et al. 2004; Pyritz et al. 2010). The behaviour of the leader of group movements plays and important role in this context; both conceptually and empirically.

Accordingly, a leader can be defined as an individual that exerts social influence on fellow group members and elicits follower behaviour (King 2010; Petit and Bon 2010; Pyritz et al. in press). Leadership can be distributed over (a subset of) all group members, or one individual can lead the group consistently (Conradt and Roper 2005; King et al. 2008; Stueckle and Zinner 2008). In general, the definition of a leader should not be restricted to its spatial position during a group movement because individuals may also lead from behind, i.e. initiate and terminate a movement without being at the forefront of the group (Kummer 1968; Pyritz et al. in press). However, in this study we use the term leader for the individual moving at the head of the group because we focus on consequences of the spatial position during movements on foraging benefits. Leadership during group movements in this sense is considered to be stable if overtaking occurs rarely during travelling, and unstable if the leading individual often changes within one single travel event (Pyritz et al. in press).

While it is already challenging to observe and analyse coordination processes of animal group movements in their natural habitat, it is even more difficult to determine the instant fitness correlates of the underlying decisions for single group members, such as individual foraging benefits (Pyritz et al. 2010). Some important insights into group foraging decisions and related feeding rates have been revealed through field experiments using artificial feeding platforms with wild capuchin monkeys (Cebus apella nigritus). These studies focused on food detection abilities depending on distance, travel speed and resource size (Janson and Di Bitetti 1997), spatial memory and strategic route planning depending on varying baiting patterns of the platforms (Janson 1998, 2007), or individual foraging benefits through deceptive vocalisation (predator alarm calls) at feeding platforms (Wheeler 2009). Only recently, experimental approaches have also been implemented in studies of coordination and decision-making regarding collective movements in (semi-)free-ranging groups (house-hunting rock ants, Temnothorax albipennis: Franks et al. 2003; honey bees, Apis mellifera: Seeley et al. 2006; sticklebacks, 
Gasterosteus aculeatus: Harcourt et al. 2009; domestic pigeons: Biro et al. 2006; Bechstein's bats: Kerth et al. 2006; white-faced capuchin monkeys, Cebus capucinus: Meunier et al. 2006; chacma baboons: King et al. 2008). In some of these experiments group decisions regarding movements to experimental feeding patches were studied. One of these experiments revealed that the spatial decisions of capuchin monkeys to move towards two mangers installed in different areas of their enclosure were mainly driven by anonymous mimetism, i.e. individuals tended to follow the travel routes previously taken by their group mates (Meunier et al. 2006). The second experiment was conducted on free-ranging chacma baboons and showed that the dominant males initiated and led all group movements to artificial feeding patches and also acquired the greatest foraging benefits. Subordinates followed in most cases despite considerable consensus costs due to social ties to the leading male (King et al. 2008).

In the present study, we experimentally investigated decision-outcomes, coordination processes, individual foraging benefits and the proximate mediation of conflict in a group of wild red-fronted lemurs (Eulemur rufifrons). Red-fronted lemurs provide an interesting model in this context for a number of reasons. First, they live in small egalitarian groups with rather equally distributed resource-holding potential (Kappeler 1991; Pereira and McGlynn 1997), providing an interesting contrast in traits influencing leadership and decision-making to taxa with larger groups or with pronounced dominance hierarchies (e.g., dwarf mongooses, Helogale parvula: Rasa 1987; plains zebras, Equus burchellii: Fischhoff et al. 2007; chacma baboons: King et al. 2008). Second, our study group is free-ranging and co-resides with a number of different predator species (Rasoloarison et al. 1995; Pyritz and Andrianjanahary 2010). This natural predation risk should favour group cohesion and consensus decision-making (van Schaik and van Noordwijk 1985). Finally, group-living evolved at least twice independently among the primates of Madagascar, compared to only once among the ancestral anthropoids (Horvath et al. 2008), on which most current coordination studies have been conducted (summarized in Fichtel et al. 2011). Thus, lemurs can provide important comparative information on the convergence of group coordination in primates from an evolutionary perspective.

We pursued two main goals in this study: (1) to measure the extent to which decisions are shared, and (2) to explore the determinants and efficiency of three potential 
tactics to decrease the consensus costs of unshared or partially shared decisions, including (i) group fission during group movement, (ii) overtaking during group movement, and (iii) aggressive supplants once the final destination is reached.

We first explored to which extent decisions were shared. To do so, we set up drinking platforms and created conflicts of interest of varying degrees by modifying baiting patterns of the platforms in two different designs, i.e. providing either several group members or only a single individual with drinking opportunities. For baiting, we used five or one bottle filled with varying amounts of flavoured water. Our predictions regarding the type of consensus decision-making at departure referred to the decision outcomes, i.e. the observed distribution of the group between the experimental platforms (Table 1): We hypothesised that unshared consensus decision-making would predominantly result in one individual leading the whole group to a platform that guarantees maximum resource intake for itself, i.e. at least one bottle filled with a maximum of flavoured water, irrespective of foraging opportunities (number of additional bottles) for followers. In contrast, shared decision-making was expected to result in the group preferably visiting platforms containing several bottles (with smaller volumes of flavoured water) instead of a platform baited with only one bottle in order to allow several individuals to drink.

Next, we explored the circumstances favouring group fissioning, predicting group fission rates - as a means to avoid costly consensus decisions in the first place by foraging in independent subgroups - (i) to decrease when the number of baited platforms was lowered in design 2 compared to design 1, and (ii) to increase after the group visited a poorly baited platform. The efficiency of group fission in decreasing consensus costs was measured by comparing the number of individuals accessing foraging rewards simultaneously in the presence versus in the absence of group fission. We expected group fission (iii) to result in more individuals drinking at the same time.

Third, we explored individual and ecological factors favouring overtaking during group movements. Our study species is characterized by stable leadership of several adult females per group under natural conditions (Erhart and Overdorff 1999; Pyritz et al. in prep.). We therefore expected adult females to predominantly initiate and lead group movements and to have the highest resource intake at the drinking platforms. In addition, we expected overtaking (=change of leadership) rate to be higher during movements 
towards one-bottle-platforms, because they only reward the first individual arriving (here considered as the leader). The efficiency of overtaking in compensating consensus costs was investigated by measuring the impact of departure and arrival order on individual resource intake at the platforms.

Finally, we explored the circumstances favouring aggressiveness at the platforms as a way to increase foraging benefits irrespective of coordination during group movements -, expecting its frequency to be negatively correlated with the number of baited platforms, which is here supposed to reflect overall resource abundance and thus the intensity of within-group feeding competition (Koenig 2002). The efficiency of aggressiveness in compensating consensus costs was measured by comparing individual resource intake after emission of aggression versus in the absence of aggression.

\section{Methods}

Study site and subjects

Data were collected at the field station of the German Primate Center (DPZ) at Kirindy Forest, a dry deciduous forest located about $60 \mathrm{~km}$ north of Morondava, western Madagascar (Sorg et al. 2003). The site is managed within a forestry concession operated by the Centre National de Formation, d'Etudes et de Recherche en Environnement et Foresterie (CNFEREF), Morondava. The forest is characterised by a pronounced seasonality with a hot wet season between December and March and a cooler dry season between April and November (Sorg and Rohner 1996). Red-fronted lemurs at Kirindy face a number of predators, such as the fossa (Cryptoprocta ferox), the harrier hawk (Polyboroides radiatus), stray dogs (Canis familiaris) and Malagasy boas (Acranthrophis ssp.) (Rasoloarison et al. 1995; Pyritz and Andrianjanahary 2010).

One group of red-fronted lemurs ("group B") regularly visited the drinking platforms. It consisted of 12 individuals ( 3 adult females, 5 adult males, 1 subadult female, 1 subadult male, 2 juvenile males; adults >2.5 years, subadults 1-2.5 years, juveniles $<1$ year). The study group is part of a habituated population inhabiting a 60 -ha area within Kirindy forest. The study area features a grid system of foot trails with intersections every $25 \mathrm{~m}$ (Fig. 1). The members of the group have been regularly captured, individually marked and observed since 1996 (e.g., Ostner and Kappeler 2004) and could therefore be distinguished easily. Relatedness of the individuals was known for 
all dyads (Wimmer and Kappeler 2002; Kappeler and Port 2008; Kappeler et al., unpub. data) except those involving 3 adult male immigrants from 2008 (BMNeg, BMPan, BMRot).

Data were collected during the peak dry season (August-September) in 2009, when most trees had shed their leaves and only a few small water holes remained in the nearby Kirindy river bed. During this time of year, several groups of red-fronted lemurs make daily forays of up to $2 \mathrm{~km}$ to reach these water holes (Scholz and Kappeler 2004), demonstrating that water represents a high-value resource for these cat-sized primates. Red-fronted lemur reproduction is highly seasonal, with matings occurring in May/June and births in September/October (Barthold et al. 2009). The adult females were therefore pregnant during this experiment.

\section{Experimental setup and designs}

We tested the experimental setup in a pilot study from July-August 2009 with a group of red-fronted lemurs that regularly visited the research camp. We attached 1 to 5 small drinking bottles (maximum volume per bottle $=250 \mathrm{ml}$ ) in bushes in the camp area in close proximity to each other (approx. $15 \mathrm{~cm}$ ). The bottles contained different volumes (10-100 $\mathrm{ml}$ ) of sweet grenadine syrup and water in a 1:10 ratio and had a light red colour. As soon as the lemurs had realized that the bottles provided drinking opportunities, they immediately moved towards them at high velocity and started drinking, indicating the high value of this experimental resource for them. During the pilot study we observed that (i) red-fronted lemurs distinguished between different syrup volumes within minutes and that they preferred bottles containing larger volumes, (ii) single individuals did not monopolize several bottles that were positioned in close proximity, and (iii) individuals did not usually displace conspecifics from other bottles after depleting their own one. We found that single lemurs always emptied volumes of $10 \mathrm{ml}$. Bottles containing $30 \mathrm{ml}$ were depleted in approx. $70 \%$ of cases. In contrast, bottles containing $\geq 75 \mathrm{ml}$ were only emptied in approx. $50 \%$ of cases. Thus, we considered $10 \mathrm{ml}$ as small, $30 \mathrm{ml}$ as medium and $75 \mathrm{ml}$ as large volumes in the experiment (Table 1).

For the actual data collection, we set up 4 drinking platforms placed $75 \mathrm{~m}$ apart from each other in the core area of group B`s home range (Fig. 1). In non-experimental observations of movements in a total of 4 groups of red-fronted lemurs between 2007 
and 2010 , all members of a group were usually detected within a $20 \mathrm{~m}$ radius $(88.8 \%$ of approx. 7600 scans of spatial group distribution). Usually, $57.6 \%$ of group members even gathered in a $10 \mathrm{~m}$ radius (Pyritz et al. 2010; Pyritz et al. in prep.). Thus, the distance of 75 $\mathrm{m}$ between the platforms was clearly more than the usual group spread, and the group had to fission in order to exploit two platforms simultaneously. Each foraging station consisted of a wooden platform with a maximum of 5 drinking bottles attached to it. Each drinking platform (including the surrounding area with a radius of approx. $3 \mathrm{~m}$ ) was monitored constantly by a custom-made surveillance system including a motion detector camera connected to a digital recorder in a waterproof box (Neumann, Ettlingen, Germany; Fig. 1). Footage of lemur visits at the platforms could be downloaded in the forest with a portable monitor and remote control.

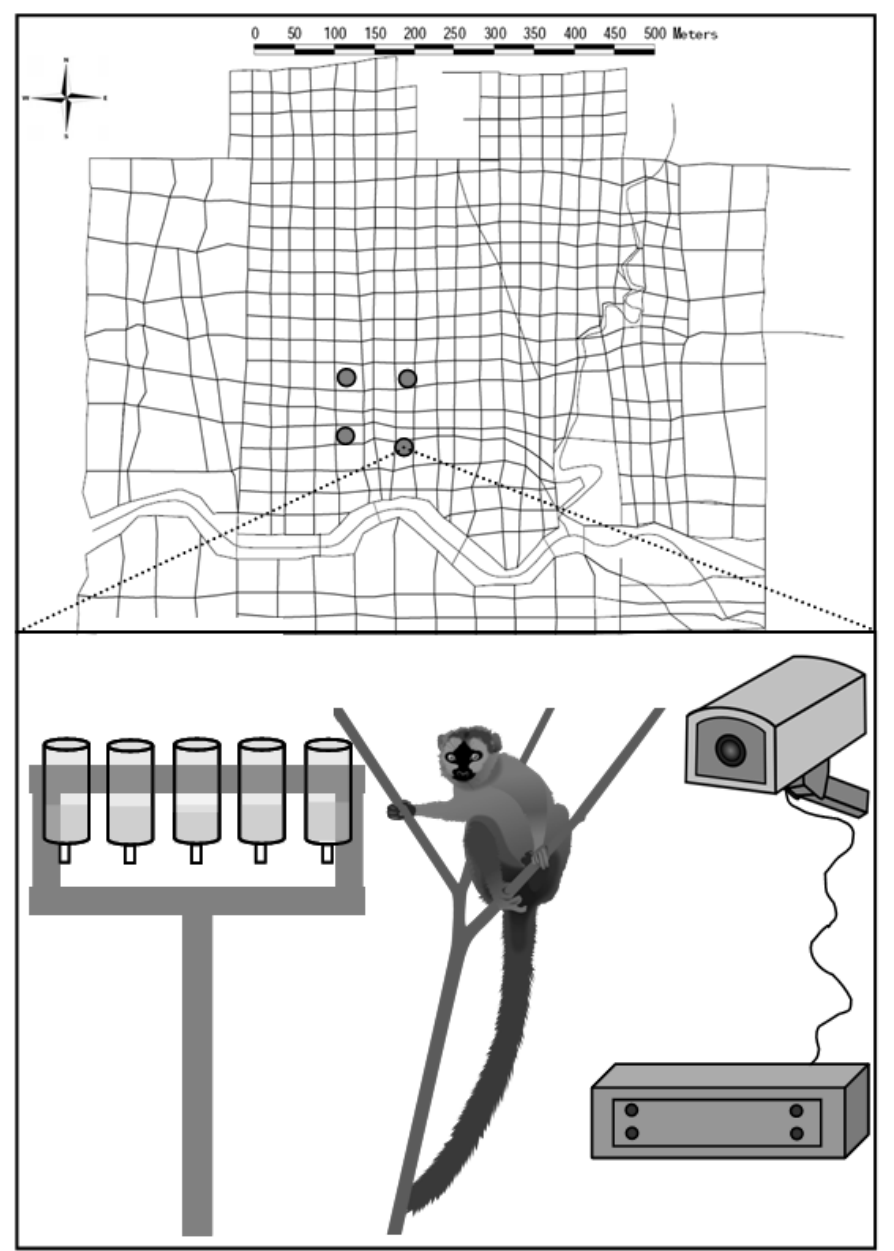

Fig. 1: Trail system of the study area at Kirindy Forest with locations of the 4 drinking platforms (above). Experimental setup: Platform with (a maximum of) 5 drinking bottles, monitored by a motion detector camera connected to a digital recorder (below). 
After a 2-week habituation phase in which all platforms were provided with 5 bottles (each containing $75 \mathrm{ml}$ ) every day, the study group visited all 4 platforms regularly, and we started data collection using the camera systems (Table 1). In the first condition of the experiment, we did not change the amount of water per bottle but provided only one bottle on platforms 1,2 and 4 in order to habituate the group to varying numbers of bottles. Data collected during this condition were not used to explore the decision type because unshared decision-making was not expected to lead to particular platforms due to an equal amount of resource in all bottles. However, data collected in condition 1 were included in tests of resource intake, group fission and aggressiveness.

Design 1 started with condition 2 (Table 1). Here, we additionally reduced the amount of water in the 5 bottles on platform 3 to $30 \mathrm{ml}$. Under these conditions, we predicted shared decision-making to result in more frequent visits to platform 3 than expected because it offers foraging opportunities for 5 group members. Conversely, unshared decision-making should bias visit rates towards the other platforms, baited each with one bottle containing $75 \mathrm{ml}$. Condition 3 equalled condition 2 but baiting patterns of platforms 2 and 3 were reversed to exclude habituation effects to platform 3.

Condition 4 marked the beginning of design 2 . On the one hand, we reduced the number of baited platforms from 4 to 2 in order to explore the consequences for group fissioning and aggressiveness at the platforms. Additionally, we reduced the resource amount at the 5-bottle-platform from medium $(30 \mathrm{ml})$ to small $(10 \mathrm{ml})$ and at the remaining 1-bottle-platform from large $(75 \mathrm{ml})$ to medium $(30 \mathrm{ml})$. We conducted these changes because we observed in the pilot study that only half of the individuals depleted $75 \mathrm{ml}$ at once, i.e. a platform baited with only 1 bottle containing $75 \mathrm{ml}$ could possibly offer foraging opportunities for at least 2 individuals, whereas bottles containing $30 \mathrm{ml}$ were depleted by 1 individual in 70\% of cases. Thus, we aimed at sharpening the conflict the group faced when making consensus decisions. As in design 1, we expected shared decision-making to result in preferred visits to the 5-bottle-platform and unshared decision-making to result in visits to the 1-bottle-platform. Condition 5 was a control equivalent to condition 3.

Each condition lasted for 1 week. However, data were recorded only during the last 4 days of each condition to give the group time to habituate to the new baiting 
pattern. During the experiment, all platforms were controlled every $30 \mathrm{~min}$. If the focal group depleted the platforms in the morning, bottles were refilled once around $2 \mathrm{pm}$. If a group other than the focal group visited the platform and drank water, bottles were refilled according to the respective experimental condition. We also refilled bottles when the identity of the group that had depleted them before was unknown. Since this unknown group later turned out to be group B quite often, $>2$ visits of the focal group at the same baited platform during one day occurred regularly (Appendix 1).

Table 1: Experimental designs and respective predictions for different types of consensus decision-making.

\begin{tabular}{|c|c|c|c|c|}
\hline Condition & Platform & $\begin{array}{l}\text { No. of } \\
\text { bottles }\end{array}$ & $\begin{array}{l}\text { Vol. per } \\
\text { bottle } \\
(\mathrm{ml})\end{array}$ & $\begin{array}{l}\text { Expected decision types for different decision } \\
\text { outcomes }\end{array}$ \\
\hline \multicolumn{4}{|c|}{ Start of data collection (Design 0 ) } & \multirow{5}{*}{$\begin{array}{l}\text { - Habituation } \\
\text { - Data included in tests on resource intake, } \\
\text { group fissioning and aggressiveness }\end{array}$} \\
\hline \multirow{4}{*}{$\begin{array}{l}\text { Condition 1: } \\
\text { Aug 4-7 }\end{array}$} & 1 & 1 & 75 & \\
\hline & 2 & 1 & 75 & \\
\hline & 3 & 5 & 75 & \\
\hline & 4 & 1 & 75 & \\
\hline \multicolumn{4}{|l|}{ Design 1} & \multirow{9}{*}{$\begin{array}{l}\text { - Shared consensus decision-making: } \\
\text { Group visits platforms baited with } 5 \text { bottles } \\
\text { more often than expected randomly. } \\
\text { - Unshared consensus decision-making: } \\
\text { Group visits platforms baited with } 1 \text { bottle more } \\
\text { often than expected randomly. }\end{array}$} \\
\hline \multirow{4}{*}{$\begin{array}{l}\text { Condition 2: } \\
\text { Aug 11-14 }\end{array}$} & 1 & 1 & 75 & \\
\hline & 2 & 1 & 75 & \\
\hline & 3 & 5 & 30 & \\
\hline & 4 & 1 & 75 & \\
\hline \multirow{4}{*}{$\begin{array}{l}\text { Condition 3: } \\
\text { Aug 18-21 }\end{array}$} & 1 & 1 & 75 & \\
\hline & 2 & 5 & 30 & \\
\hline & 3 & 1 & 75 & \\
\hline & 4 & 1 & 75 & \\
\hline \multicolumn{4}{|l|}{ Design 2} & \multirow{9}{*}{$\begin{array}{l}\text { - Shared consensus decision-making: } \\
\text { Group visits platform baited with } 5 \text { bottles more } \\
\text { often than expected randomly. } \\
\text { - Unshared consensus decision-making: } \\
\text { Group visits platform baited with } 1 \text { bottle more } \\
\text { often than expected randomly. } \\
\text { - Test effects of the number of baited platforms } \\
\text { on group fissioning and aggressiveness }\end{array}$} \\
\hline \multirow{4}{*}{$\begin{array}{l}\text { Condition 4: } \\
\text { Aug 25-28 }\end{array}$} & 1 & - & - & \\
\hline & 2 & 5 & 10 & \\
\hline & 3 & 1 & 30 & \\
\hline & 4 & - & - & \\
\hline \multirow{4}{*}{$\begin{array}{l}\text { Condition 5: } \\
\text { Sep 1-4 }\end{array}$} & 1 & - & - & \\
\hline & 2 & 1 & 30 & \\
\hline & 3 & 5 & 10 & \\
\hline & 4 & - & - & \\
\hline
\end{tabular}

\section{Data collection}

Data were collected between August 4 and September 4 (Table 1), with the camera system operating throughout the daylight period, from 6:15-17:00, on each day of the experiment. All visits of the study group recorded during this time $(n=110$; approximate 
total duration: $10 \mathrm{~h}$ ) were analysed using EverFocus Player MFC Application 2008 (EverFocus Electronics Corporation, Emmerich am Rhein, Germany). Data extracted from the videos included number of daily visits per platform, total number of individuals visiting the platform and individual length of stay, arrival order, individual resource intake and agonistic interactions at the platforms. Individual resource intake could be assessed accurately from the videos due to the light red colour of the flavoured water and highlighted measuring lines on the drinking bottles. For agonistic interactions (bite, chase $>2$ m, cuff, grab: Pereira and Kappeler 1997), each event was counted separately because one bite or cuff could possibly supplant an opponent from a bottle and offer a drinking possibility. A fission event was counted if 1 or more individuals of the study group were recorded at different platforms at the same time.

Behavioural observations were also conducted during 17 days (85\%) of the experiment total of $81 \mathrm{~h}$ ). The observer recorded every group movement according to an operational definition developed during a pilot study (Pyritz et al. 2010; Fichtel et al. 2011). We recorded data on initiation, followership and overtaking in a total of 25 movements that terminated at a drinking platform. However, these movements often proceeded fast and with multiple change of leadership, making it difficult to protocol each detail. Therefore, we assessed overtaking events during most movements retrospectively by checking if the initiator of a movement also arrived first at the drinking platform on the corresponding video. If the initiator arrived $\geq 5 \sec$ (usually corresponding to several body lengths) after the first individual at the platform, this was counted as an overtaking event.

\section{Data analyses}

We used a Mann-Whitney U-test (MWU) to explore whether the number of bottles per platform ( 1 or 5 ) affected the number of individuals per visit, i.e. whether more bottles indeed provided more individuals with a foraging opportunity. Whether group fissioning was beneficial in terms of more individuals drinking simultaneously was also evaluated with a MWU test. In order to identify the type of consensus decision-making, we calculated Pearson`s chi-squared tests with Yate`s continuity correction (corrected for the different proportions of 1- and 5-bottle-platforms in designs 1 and 2) to compare the number of non-fission visits to platforms baited with 5 and 1 bottle, respectively. We used 
Fisher's exact tests to assess whether the study group preferred particular platforms, irrespective of baiting patterns in design 1 and 2. We used Fisher's exact and a chisquared tests to determine whether overtaking increased with the number of bottles on the platform the movement was directed to (corrected for proportions of observed group movements towards platforms baited with 1 bottle and 5 bottles, respectively) and to compare overtaking rates during the experiment to observations of the same group from non-experimental contexts (Pyritz et al. in prep.), respectively. Impacts of baiting patterns on fissioning were analyzed using a chi-squared and Fisher's exact test, too. All tests and figures were generated with PASW Statistics 18 (SPSS Inc., Chicago, IL, 2009).

Table 2: Parameter estimates for the most parsimonious linear mixed models (LMM) on determinants of resource intake.

\begin{tabular}{|c|c|c|c|c|c|c|}
\hline Model & $\begin{array}{l}\text { Response } \\
\text { variable }\end{array}$ & $\begin{array}{l}\text { Random } \\
\text { factors }\end{array}$ & Fixed factors & Estimate & $\overline{\mathrm{SE}}$ & P-value \\
\hline \multirow[b]{2}{*}{$\begin{array}{l}\text { At departure } \\
\text { LMM } \\
\text { (intakes of } \\
\text { individuals } \\
\text { observed during } \\
\text { movements to } \\
\text { platforms; } n=31 \text { ) }\end{array}$} & \multirow[b]{2}{*}{$\begin{array}{l}\text { Individual } \\
\text { resource } \\
\text { intake at } \\
\text { platforms } \\
\text { (sqrt \%) }\end{array}$} & \multirow{2}{*}{$\begin{array}{l}\text { Animal ID, } \\
\text { number of } \\
\text { bottles per } \\
\text { platform }\end{array}$} & Intercept & 3.40 & 1.16 & $<0.01^{*}$ \\
\hline & & & $\begin{array}{l}\text { Departure position } \\
\text { (leader, follower) }\end{array}$ & -0.10 & 1.39 & 0.94 \\
\hline \multirow{5}{*}{$\begin{array}{l}\text { At arrival } \\
\text { LMM } \\
\text { (intakes of all } \\
\text { individuals } \\
\text { arriving at } \\
\text { platforms; } \\
n=204 \text { ) }\end{array}$} & \multirow{5}{*}{$\begin{array}{l}\text { Individual } \\
\text { resource } \\
\text { intake at } \\
\text { platforms } \\
\text { (sqrt \%) }\end{array}$} & \multirow{5}{*}{$\begin{array}{l}\text { Animal ID, } \\
\text { number of } \\
\text { bottles per } \\
\text { platform }\end{array}$} & Intercept & 5.18 & 1.53 & $<0.001 * * *$ \\
\hline & & & Arrival order & -1.04 & 0.12 & $<0.001^{* * *}$ \\
\hline & & & $\begin{array}{l}\text { Visit number per } \\
\text { platform per day }\end{array}$ & & & No effect \\
\hline & & & $\begin{array}{l}\text { Age } \\
\text { (adult, subadult) }\end{array}$ & & & No effect \\
\hline & & & $\begin{array}{l}\text { Sex } \\
\text { (female, male) }\end{array}$ & & & No effect \\
\hline
\end{tabular}

Significance level is at 0.05. sqrt=square-root-transformed data, ID=identity, SE=standard error, No effect=factor tested but not part of the model with the best fit

In order to analyse determinants of resource intake and aggressiveness, we calculated three linear mixed models (LMM) and one generalized linear mixed model (GLMM; Zuur et al. 2009). We chose these models instead of simpler statistics because they allowed exploring influences of several explanatory variables simultaneously while controlling for repeated observations of the same animals, as well as for artificial variance resulting from a variable number of bottles per platform. Individual identity and number of bottles per platform were fitted as crossed random factors. We chose number of 
bottles as random factor rather than total resource volume per platform because bottles represented the monopolisable units for the individuals.

Table 3: Parameter estimates for the most parsimonious (generalized) linear mixed model (GLMM and LMM) on determinants and benefits of aggressiveness.

\begin{tabular}{|c|c|c|c|c|c|c|}
\hline Model & $\begin{array}{l}\text { Response } \\
\text { variable }\end{array}$ & $\begin{array}{l}\text { Random } \\
\text { factors }\end{array}$ & Fixed factors & Estimate & SE & P-value \\
\hline \multirow{7}{*}{$\begin{array}{l}\text { Determinants of } \\
\text { aggressiveness } \\
\text { Quasi-poisson } \\
\text { GLMM ( } \geq 2 \\
\text { individuals } \\
\text { per platform; } \\
n=147 \text { ) }\end{array}$} & \multirow{7}{*}{$\begin{array}{l}\text { Counts of } \\
\text { aggressions } \\
\text { emitted }\end{array}$} & \multirow{7}{*}{$\begin{array}{l}\text { Animal ID, } \\
\text { number of } \\
\text { bottles per } \\
\text { platform }\end{array}$} & Intercept & -5.72 & 0.41 & $<0.001^{* * *}$ \\
\hline & & & $\begin{array}{l}\text { Number of } \\
\text { individuals } \\
\text { on platform }^{1}\end{array}$ & 0.48 & 0.03 & $<0.001^{* * *}$ \\
\hline & & & $\begin{array}{l}\text { Time on platform } \\
\text { (log sec) }\end{array}$ & 1.50 & 0.13 & $<0.001^{* * *}$ \\
\hline & & & $\begin{array}{l}\text { Age (adult, } \\
\text { subadult) }\end{array}$ & -1.36 & 0.25 & $<0.05^{*}$ \\
\hline & & & $\begin{array}{l}\text { Visit number per } \\
\text { platform per day }\end{array}$ & & & No effect \\
\hline & & & $\begin{array}{l}\text { Sex (female, } \\
\text { male) }\end{array}$ & & & No effect \\
\hline & & & $\begin{array}{l}\text { Number of } \\
\text { baited platforms }\end{array}$ & & & No effect \\
\hline \multirow{5}{*}{$\begin{array}{l}\text { Benefits of } \\
\text { aggressiveness } \\
\text { LMM } \\
\text { ( } \geq 2 \text { individuals } \\
\text { per platform; } \\
n=147 \text { ) }\end{array}$} & \multirow{5}{*}{$\begin{array}{l}\text { Individual } \\
\text { resource } \\
\text { Intake at } \\
\text { platforms } \\
\text { (sqrt \%) }\end{array}$} & \multirow{5}{*}{$\begin{array}{l}\text { Animal ID, } \\
\text { number } \\
\text { of bottles } \\
\text { per } \\
\text { platform }\end{array}$} & Intercept & 3.17 & 0.59 & $<<0.001^{* * *}$ \\
\hline & & & $\begin{array}{l}\text { Individual } \\
\text { aggression } \\
\text { rates at platforms } \\
\text { (events/hour) }\end{array}$ & -0.00 & 0.01 & 0.58 \\
\hline & & & $\begin{array}{l}\text { Visit number per } \\
\text { platform per day }\end{array}$ & & & No effect \\
\hline & & & $\begin{array}{l}\text { Sex (female, } \\
\text { male) }\end{array}$ & & & No effect \\
\hline & & & $\begin{array}{l}\text { Age (adult, } \\
\text { subadult) }\end{array}$ & & & No effect \\
\hline
\end{tabular}

Significance level is at 0.05. log=log-transformed data, sqrt=square-root-transformed data, $I D=i d e n t i t y, S E=s t a n d a r d$ error, No effect=factor tested but not part of the model with the best fit

${ }^{1}$ Number of group members that were present on the platform $\geq 50 \%$ of the time with the individual. Times that an individual spent on the platform alone were excluded from the analysis.

Furthermore, number of bottles and total resource volume per platform were highly correlated (Pearson correlation coefficient: $n=20 ; r=0.62, p<0.01$ ). Tables 2 and 3 provide an overview of data distribution, error structure and explanatory variables of the models. The first model in Table 2 explored whether departure order explained resource intake at a platform and was based on the observations of group movements towards the 
platforms. The second model was based on data from the video recordings and assessed the impact of arrival order, sex and age on resource intake, including visits where only one individual had been recorded at a platform. Additionally, we included visit number per day as fixed factor to account for repeated visits. In the first model in Table 3, we analysed which factors (sex, age, number of individuals, visit number, number of baited platforms) influenced aggressiveness. We used count data of emitted aggressive interactions (Poisson distribution with overdispersion, fitted with a quasi-Poisson GLMM) as response variable and controlled for the time spent on the platform as a fixed factor. The second model explored whether individual resource intake at the platforms was affected by the individual aggression rates, again taking individual age, sex and number of visits at the same platform per day into consideration. For both models on aggressiveness, we used only visits where $\geq 2$ individuals were present at a platform.

Models were fitted using R software (R Development Core Team, Vienna, Austria, 2010) with the Ime4-package. For models with normal error structure, we used Akaike's Information Criterion (Akaike 1974) to remove parameters in a step-wise fashion in order to select the most parsimonious model with the best fit. Factors were excluded only if this improved the model fit by $>2$ AIC units (Mundry and Nunn 2009). We used maximum likelihood ratio tests to test whether a fixed factor explained a significant amount of the variance in the presence of the other factors and to test the final model with fixed factors against the null model including only the random factors (Faraway 2006). In case of overdispersion, we corrected standard errors using a quasi-GLM-model (Zuur et al. 2009). Because in this model the AIC is not defined, we used chi-squared tests with the deviances of two models differing in only one fixed factor to test the goodness of fit and select the most parsimonious model (Faraway 2006). We used square-root and logtransformation on some variables to improve the model fit (Zuur et al. 2009). The significance level was set at 0.05 .

\section{Results}

General responses to the experimental setup

All baited platforms were visited frequently throughout the experiment with individual platforms being visited 0-4 times per day (Appendix: Table A1). Each group member visited the four platforms and drank more than 10 times in total, except for 2 adult males 
(BMGig: 3 visits; BMNeg: 8 visits) and 1 subadult male (BMRut: 9 visits). Individuals arriving at a platform emptied at once the $75 \mathrm{ml}$-bottles in 44\%, the $30 \mathrm{ml}$-bottles in $84 \%$ and the $10 \mathrm{ml}$-bottles in $100 \%$ of visits. Only rarely were more than $25-50 \%$ of group members visible at the platforms on the video recordings (Fig. 2). However, the number of individuals recorded at a platform increased significantly with the number of bottles per platform (MWU test: 1 bottle: $n=60,2.3 \pm 1.5$; 5 bottles: $n=40,3.7 \pm 2.6$; $U=768.0, z=-$ $3.78, p<0.01)$.

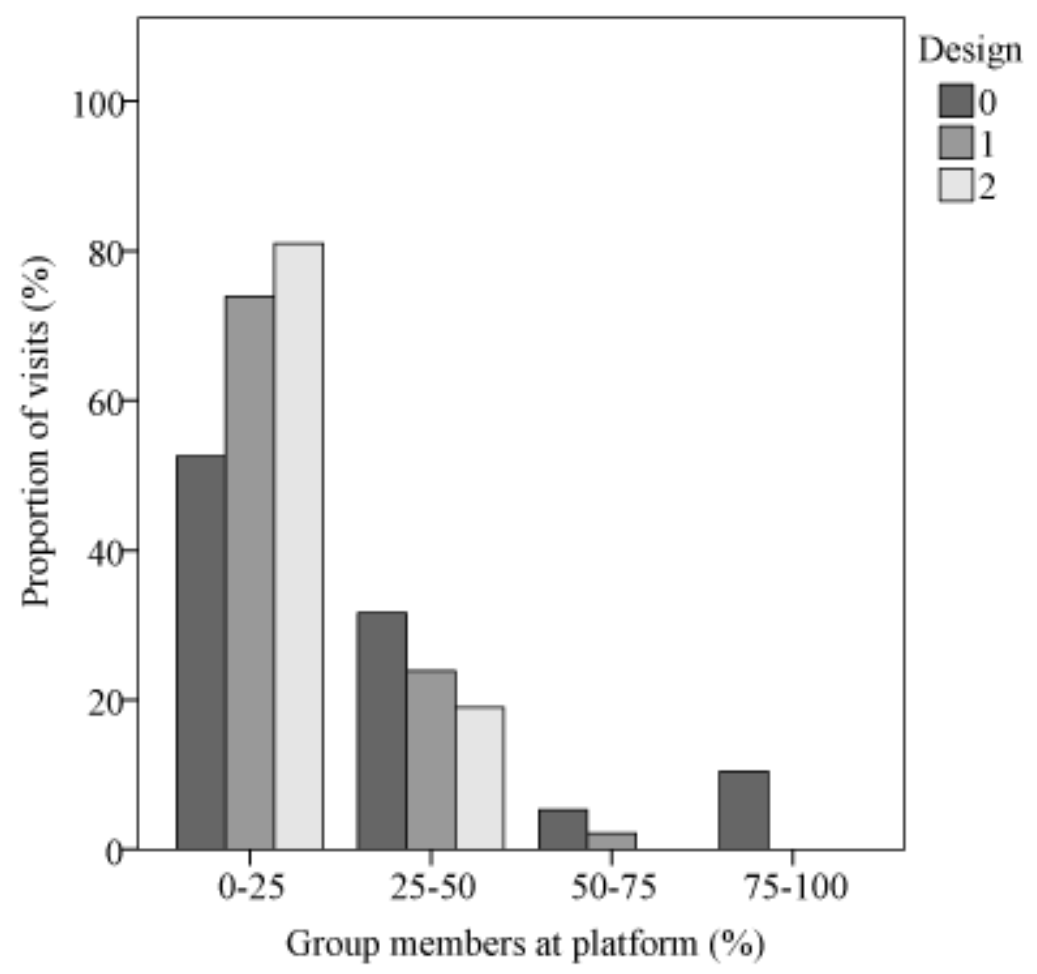

Fig. 2: Proportion of group members (in percent) that was visible on the video recordings during platform visits throughout different experimental designs $(N=110)$.

\section{Consensus decision-making}

The number of non-fission visits to platforms baited with 5 bottles compared to platforms baited with only 1 bottle did not differ from random expectations in either design (chisquared tests: design $1: \mathrm{n}=27 ; \chi^{2}=0.11, \mathrm{df}=1, \mathrm{p}=0.74 ;$ design $2: \mathrm{n}=37 ; \chi^{2}=1.32, \mathrm{df}=1$, $p=0.25$ ). Therefore, we were not able to assess whether consensus decision-making was shared or unshared based on the decision outcomes observed during the experiment. In fact, the group visited platform 3 more often than the other platforms in each condition, i.e. independent of baiting patterns (non-fission visits to baited platforms). However, 
differences between the visit numbers at platforms were not significant compared to random expectations (condition 1-3: 25\% visits per platform expected; conditions 4-5: $50 \%$ visits for platforms 2 and 3 expected; Fisher's exact tests: condition $1: n=15 ; p=0.11$; condition 2 : $n=11 ; p=0.30$; condition $3: n=17 ; p=0.49$; condition $4: n=12 ; p=1.00$; condition 5: $n=16 ; p=0.72$ ).

\section{Group fissions}

The group split into subgroups during 12 out of 110 visits recorded at the platforms (10.9\%). Ten times, 2 subgroups were recorded at different platforms, two times 3 subgroups. Subgroup size ranged from 1 to 8 , with a mean of $2.3 \pm 1.7$ individuals. The proportion of group fissions compared to non-fission visits was larger when 4 platforms were baited (17.6\%) instead of only 2 platforms (7.5\%) but the difference was not significant (Fisher's exact test: $n=96$, fissions counted as one event; $p=0.22$ ). The group did not split into subgroups more often after visiting a 1-compared to a 5-bottleplatform, either (chi-squared test: $n=86$, fission visits and visits to non-baited platforms excluded; $\chi^{2}=0.06, d f=1, p=0.81$; controlled for total number of visits to platforms baited with 1 and 5 bottles, respectively). However, when the group fissioned, on average more individuals drank simultaneously $(2.0 \pm 0.8)$ than during non-fission visits $(1.3 \pm 1.4$; MWU: $\mathrm{n}_{\text {fission }}=12, \mathrm{n}_{\text {non-fission }}=84 ; \mathrm{U}=337.5, \mathrm{z}=-2.78, \mathrm{p}<0.01$ ).

\section{Group coordination, overtaking and resource intake}

Observed movements towards drinking stations $\left(n_{\text {total }}=25 ; n_{\text {with followers }}=15\right)$ were initiated by adults of both sexes and by one subadult male, with the oldest female of the group being responsible for $72 \%$ of all initiations. This high proportion of female initiatorship does not differ from observations of natural group movements of the same study group (Pyritz et al. in prep.; chi-squared test: $n_{\text {exp }}=25, n_{\text {other }}=251 ; \chi^{2}=0.11, d f=1, p=0.74$ ). However, overtaking rates during the experiment (67\%) were significantly higher than expected from movements not directed at foraging platforms of the same group during the same time period ( $18 \%$; chi-squared test: $n_{\exp }=15, n_{\text {other }}=57 ; \chi^{2}=47.1, d f=1, p<0.001$; Fig. 3). We observed individuals of all sex and age classes overtaking throughout all conditions. However, overtaking rate did not decrease with the number of bottles on the platform visited (Fisher's exact test: $n=10 ; p=1.00$ ). Departure position, i.e. whether an 
individual initiated or followed a movement, did not affect individual resource intake (Table 2) since the full model did not explain significantly more variance than the null model ( $L M M: n=31 ; \chi^{2}=2.20, d f=2, p=0.33$ ). In contrast, arriving first was - in general advantageous in terms of foraging benefits (Table 2; Fig. 4) and the model differed significantly from the null model ( $\left.L M M: n=204 ; \chi^{2}=56.33, d f=1, p<0.001\right)$. Visit number per platform per day, sex and age did not contribute significantly to the final model (Table 2).

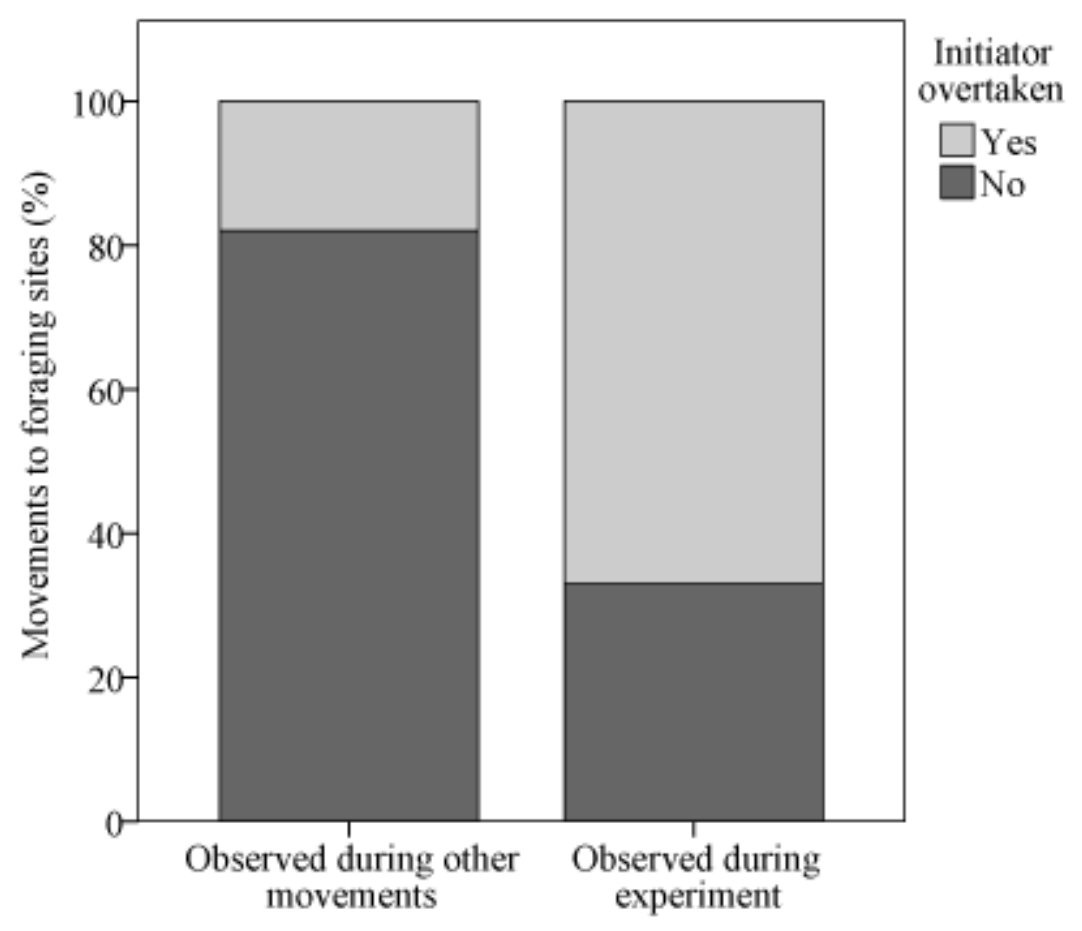

Fig. 3: Overtaking events (in percent) during movements towards drinking platforms compared to other foraging movements of the group observed during the same time period. $N=15$ for experiment, $N=57$ for other observations

\section{Social interactions at the platforms}

Agonistic interactions (total number observed on the video recordings=111) occurred during 30 out of 54 visits of $\geq 2$ individuals at a baited platform (55.6\%). In 8 cases (7.2\%), a single agonistic action (bite, cuff, grab) resulted in the direct displacement of a conspecific from a bottle and offered a foraging opportunity for the aggressor. Individuals that had previously overtaken the leader were never displaced from a bottle. Aggressive interactions were more likely to happen when more individuals were present at a platform and when an individual spent more time at the platform. Furthermore, adults 
showed more aggressive behaviour than subadults. In contrast, sex, visit number per day and the number of baited platforms did not contribute to the most parsimonious model (Table 3). The final model performed significantly better than the null model (quasipoisson GLMM: $n=147$ visits with $\geq 2$ individuals; $\chi^{2}=55.45, d f=4, p<0.001$ ). However, a higher aggression rate did not result in increased resource intake. Visit number, age or sex did not contribute to the model with the best fit and the final model did not have a better fit than the null model (LMM: $n=147$ visits with $\geq 2$ individuals; $\chi^{2}=0.31, d f=1$, $\mathrm{p}=0.58)$.

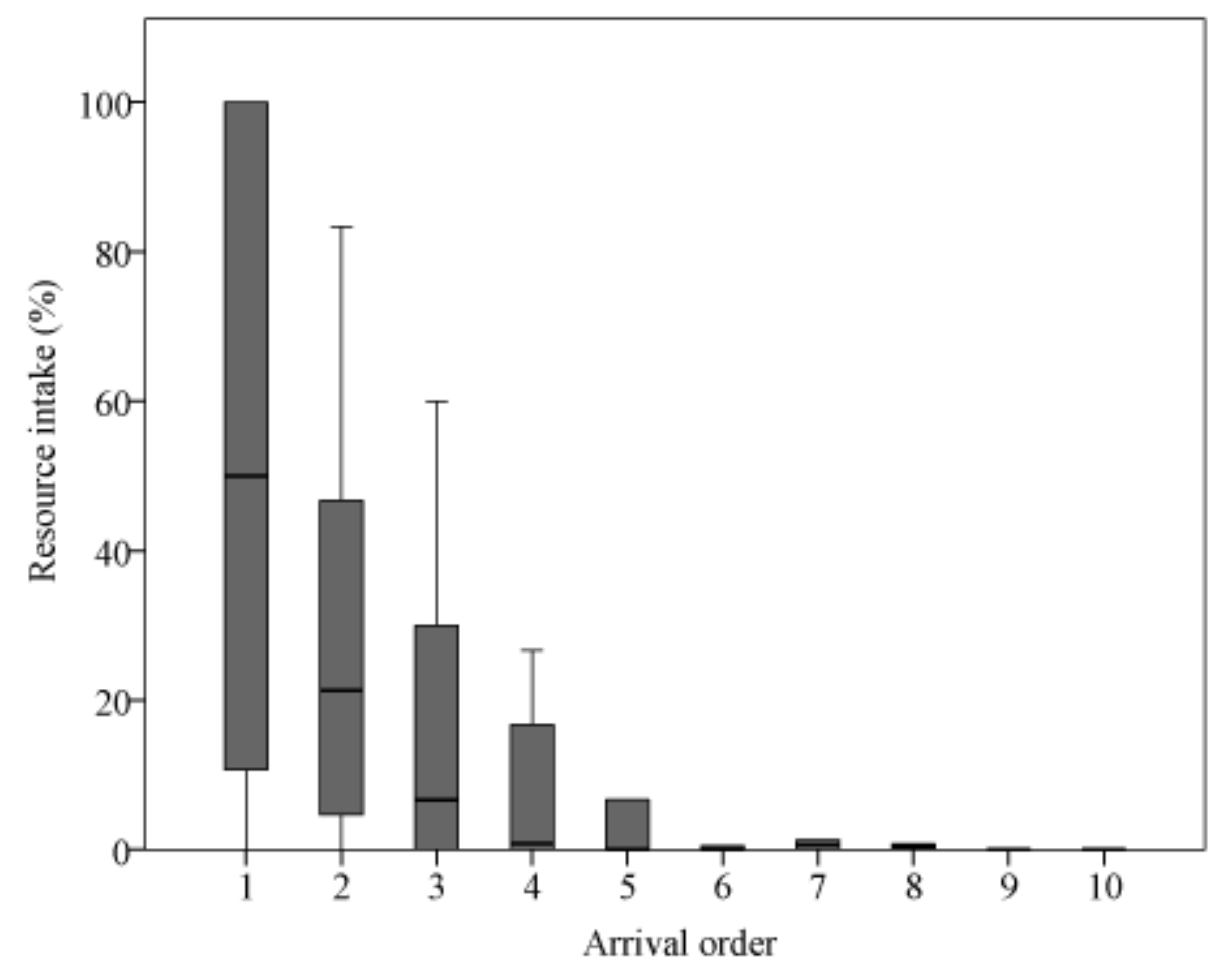

Fig. 4: Individual resource intake (in percent of total volume available at the platform) in relation to arrival order. Shown are medians, $25-75 \%$ quartiles (box) and ranges (whiskers). $N=204$

\section{Discussion}

Although these data are based on observations of a single group (see also Janson and Di Bitteti 1997; Janson 2007; Wheeler 2009), our study provides important insights into the variability of coordination processes and related consequences for individual foraging benefits in a free-ranging primate group. Red-fronted lemurs reacted to changes in the experimental design in terms of more individuals visiting the platforms when more bottles were provided. However, the number of visits per platform did not depend on 
baiting patterns. Therefore, we were not able to identify the decision type based on the observed decision outcomes. Adult females initiated most movements towards the platforms. However, in contrast to observations from natural contexts, change of leadership was more frequent. Accordingly, initiators did not have a higher resource intake than followers but foraging benefits depended on arrival order. Group fissions resulted in more individuals drinking simultaneously. Fissioning was not affected by changes in baiting patterns, however. Individual aggression rates did not affect foraging benefits in terms of higher resource intake. We discuss each of these main findings in more detail below.

\section{General responses to the experimental setup}

Most individuals of our study group visited the platforms and depleted the experimental resource regularly, which was a prerequisite for the foraging experiment and signals a high value of the resource. The proportions of different resource volumes depleted by single individuals during the experiment were similar to those assessed for the camp group during the pilot study on which we based our experimental conditions. However, usually only a few group members were visible at the platforms on the video recordings. This could be a result of group fissions during which only one subgroup visited a platform. Unfortunately, we did not capture these fission events with our definition that relied on two subgroups being video-recorded at different platforms at the same time. On the other hand, the number of individuals recorded increased significantly with the number of bottles at the platforms, which might indicate that the group was usually present around the platform and a larger number of group members only jumped onto the platform, i.e. inside the camera frame, when more than one bottle was present. It also shows that more bottles provided indeed more individuals with a foraging opportunity, i.e. single individuals did not monopolise entire platforms.

\section{Consensus decision-making}

We tried to identify whether decision-making in groups of red-fronted lemurs was shared or unshared using platforms that provided either one or several group members with a foraging opportunity. Our predictions were based on observations during the pilot study that included rapid distinguishing between different resource volumes in the bottles and 
high group cohesion. However, the number of group visits to a platform was obviously not affected by baiting patterns, and we were not able to identify the decision type underlying the observed decision outcomes. There are a number of reasons that might have hampered our approach.

1. It was difficult to determine the resource volume that offered a sufficient foraging benefit for exactly one individual. For instance, the random visiting patterns observed in design 2 could be due to unshared decision-making if the decision-maker always drank more than $30 \mathrm{ml}$, i.e. led the group also to the 5-bottle-platform after depleting the 1-bottle-platform. Furthermore, we offered a maximum of 5 foraging opportunities. However, decision-making might be partially shared with only 2-3 individuals contributing to the outcome (e.g., Sueur and Petit 2008a, b). Under these circumstances, a 1-bottle-platform offering $75 \mathrm{ml}$ could already have provided foraging benefits for all individuals involved in the decision-making since they could drink subsequently from the same bottle.

2. The lemurs in the pilot study rapidly distinguished between different volumes of single bottles. However, the final experimental setup comprising four platforms was much more complex. Therefore, the lemurs may have failed to react towards variation in baiting patterns because we changed between different conditions too fast throughout the experimental designs.

3. The concept of discrete, monopolisable resource units (bottles) that have the same size but offer different foraging benefits could lack ecological relevance because redfronted lemurs usually feed on dispersed fruit and leaves under natural conditions (Sussman 1974; Overdorff 1993a).

Alternatively, it is also possible that consensus decision-making does not present the only means to optimize group foraging benefits. For instance, group members can avoid consensus costs through different strategies such as (1) breaking group cohesion, which can presumably maximize the benefits of a majority of individuals (see e.g., Kerth 2010), (2) shifting the benefits of consensus decision-making by overtaking initiators or aggressively displacing group members that arrived earlier at the resource. We tried to identify the determinants and outcomes of each of these possibilities and subsequently discuss the respective results. 


\section{Group fissions}

Regarding determinants of group fissions, we predicted that fission probability would increase with the number of baited platforms and after the group visited a poorly baited platform. None of these hypotheses was supported, suggesting that baiting patterns do not affect group fissions. However, we cannot exclude the possibility that our operational definition of fission, which was constrained by the use of automatic cameras, might have captured only a subset of the real fission events. Unfortunately, we could not compare fission rates during the experiment (= inter-individual distances of $75 \mathrm{~m}$ ) to natural contexts because respective data on inter-individual distances $>20 \mathrm{~m}$ under natural conditions are lacking.

In accordance with our third prediction, fissioning resulted in a significantly higher number of individuals drinking from bottles at the same time. Hence, fissioning apparently allowed group members to avoid costly consensus decisions and provided instant foraging benefits for a higher number of individuals (Kerth 2010). Such temporary splitting into subgroups as a consequence of high consensus costs has been observed in a number of species, including pigeons (Biro et al. 2006), bats (Kerth et al. 2006) and baboons (King et al. 2008). However, there are also potential costs of group fissions. Decreased cohesion should negatively affect shared vigilance and predator confusion and, thus, increase predation risk (Bertram 1978; Fichtel in press). This may be particularly salient during the dry season when we conducted the experiment because during this time Madagascar's largest carnivore, the fossa, mainly feeds on lemurs (Hawkins and Racey 2008), and predation risk is supposed to be high due to defoliated trees (Janson and Goldsmith 1995). Thus, benefits of group fissions in terms of enhanced resource access supposedly outweighed the costs of increased predation risk during the experiment (see also Houston et al. 1993 and Moody et al. 1996 for theoretical overviews on energy-risk trade-offs).

\section{Group coordination, overtaking and resource intake}

We predicted predominantly stable leadership by adult females towards the platforms and, accordingly, highest foraging benefits for these individuals. Consistent with our predictions, adult females initiated most movements towards the platforms. Female initiator- and leadership has been described in a number of mammal species (dwarf 
mongooses: Rasa 1987; Grevy`s zebras, Equus grevyi, and onagers, Equus hemionus: Rubenstein 1994; plains zebras: Fischhoff et al. 2007; primates: summarized in Fichtel et al. 2011) and is usually attributed to the high energetic needs of females during gestation and lactation. Since we conducted the experiment during the gestation period of redfronted lemurs, females might have initiated group movements in order to compensate increased physiological requirements as well.

However, female leadership was not stable. In fact, overtaking by individuals of all age and sex classes occurred during two-thirds of movements throughout all experimental conditions. During natural movements, change of leadership occurred in only $18 \%$ of cases, and no overtaking at all was reported in another study on group movements in red-fronted lemurs by Erhart and Overdorff (1999). As a consequence of overtaking, initiators did not arrive first at the drinking platforms, and arrival instead of departure order predicted individual foraging benefits. Neither sex nor age affected resource intake, and it was not always the same individuals who arrived at a platform first. We expected higher overtaking rates during movements towards 1-bottle-platforms. However, the number of bottles did not determine change of leadership, which is in line with the observation that baiting patterns did not affect visit numbers at platforms, either.

Overtaking rates in the present study were apparently affected by the high value and predictability of the experimental resource compared to natural foraging patches. However, such changes in coordination processes have not been described in comparable studies involving movements to experimental foraging patches by groups of white-faced capuchin monkeys (Meunier et al. 2006) and chacma baboons (King et al. 2008). These species are characterized by clear dominance hierarchies that have been shown to result in asymmetric resource holding potential (Koenig 2002; Vogel 2005). Therefore, the high frequency of overtaking by individuals from all age and sex classes observed here could be facilitated by the egalitarian social relationships among red-fronted lemurs (Kappeler 1991; Pereira and McGlynn 1997). In fact, we never observed an individual that had previously overtaken being punished by other group members after arrival on the platforms. 


\section{Social interactions at the platforms}

Fissioning offered the possibility to avoid costly consensus decision-making in the first place. Overtaking resulted in earlier arrival at the platforms and higher resource intake. A third tactic to optimise individual foraging benefit could be supplanting of group members from bottles through aggressive behaviour at the platforms. As in other studies on red-fronted lemurs (Pereira et al. 1990; Erhart and Overdorff 1999), we observed aggressive behaviour during feeding throughout the experiment. Aggression increased with the number of individuals and the time spent on the platform. Furthermore, adults emitted aggression more often than subadults. However, contrary to our hypothesis, the frequency of agonistic interactions at the platforms was not higher on platforms with fewer bottles.

At first sight, higher aggression levels of adults compared to subadults seem to reflect the proximate importance of physical asymmetry in accessing a food resource (Pereira 1988; Gompper 1996). However, aggression resulted in the displacement of a conspecific from a bottle in only 7.2\%. Therefore, aggression was not a powerful mechanism to obtain a foraging opportunity but rather a consequence of increased agitation or opportunities for cuffs and grabs due to spatial crowding (Erwin and Erwin 1976). The assumption of high social tolerance exhibited by adults towards subadults at the platforms is also in line with another observation: mothers and their offspring were observed to drink simultaneously from the same bottle for prolonged time spans (>5 sec) in 22 cases.

\section{Conclusions}

The skew of foraging opportunities at artificial drinking platforms did not affect the outcomes of consensus decision-making in red-fronted lemurs and the decision type was undetermined. In fact, red-fronted lemurs evaded the foraging costs of group cohesion and stable leadership by regular fissioning into subgroups or overtaking the initiator of a movement, which proved beneficial in terms of both higher collective and individual resource intake. However, aggression at the platforms was not a useful tactic to augment individual foraging benefits. Our study also permits a number of more general conclusions regarding the relationships among coordination, foraging behaviour and social structure in animal groups. First, costs of a temporary lack of coordinated cohesion can be 
outweighed by high resource quality and predictability, even under supposedly high predation risk. Second, coordination in animal groups characterised by an egalitarian social structure can be highly flexible, allowing the use of alternative behavioural tactics that translate into individual foraging benefits irrespective of sex, age or social status.

\section{Acknowledgements}

We acknowledge the authorization of this study by the Département de Biologie Animale, Universite d'Antananarivo, the CAFF of the Direction des Eaux et Forêts and the CNFEREF Morondava. For assistance in the field we are deeply grateful to Jean-Pierre Tolojanahary, Patrick de Beroboka, Tianasoa Andrianjanahary and the other members of the Equipe Kirindy. Lothar Neumann and Manfred Eberle helped realising the study with their technical expertise. This manuscript benefited greatly from comments by Dagmar Clough, Cornelia Kraus, Cédric Sueur and discussions with the participants of the symposium "Where next? Coordination and decision making in primate groups" at the XXIII Congress of the International Primatological Society (IPS). The German Research Foundation (DFG: KA 1082/16-1) provided financial support. 


\title{
CHAPTER 5
}

Collective Mobbing of a BoA by a GRoup of Red-Fronted LeMURS (EULEMUR RUFIFRONS)

\author{
with Tianasoa Andrianjanahary
}

Lemur News 15:15-17 (2010) 


\section{Introduction}

Collective anti-predator behaviour is one of the principal advantages of group-living (for mammals, e.g., Janzen 1970; van Schaik 1983). It can be broadly divided into two strategies and tactics employed before and after predator encounters (Caro 2005; Rahlfs and Fichtel 2010; Fichtel, in press). While the former include predator-sensitive foraging and increased vigilance, mobbing occurs in several mammal species after detecting a predator (e.g., Tamura 1989). Why animals engage in mobbing and who benefits from it in which way remains an unresolved question in animal behaviour (for reviews see Curio et al. 1978; Shields 1984). Until today, published field observations of group-living lemurs mobbing a predator are rare (summarised in Scheumann et al. 2007). Regarding snakes, only three interactions have been described so far (Colquhoun 1993; Rakotondravony 1998; Burney 2002). Here, we report a prolonged mobbing display against a Madagascar ground boa (Acrantophis madagascariensis) by a group of red-fronted lemurs (Eulemur rufifrons) in Kirindy Forest. Observations like this may help to elucidate fundamental mechanisms of collective anti-predator behaviour by contributing to a pool of data on mobbing by particular pairs of prey and predators.

\section{Observations}

The event was observed during regular behavioural observations of red-fronted lemurs in Kirindy Forest, $60 \mathrm{~km}$ north of Morondava. It was the only snake-lemur interaction observed during the entire study period from November 2007 to April 2010, in which four lemur groups were followed daily by one or two observers, respectively $(>4,000 \mathrm{~h}$ of observation data). Red-fronted lemurs live in multi-male-multi-female groups of 5-12 individuals (Pereira and Kappeler 1997; Wimmer and Kappeler 2002). The study group (B) that encountered the boa included 9 individually marked animals at the time $(2$ adult females, 5 adult males, 1 juvenile male, 1 male infant).

On March 1, 2010, at $7.19 \mathrm{~h}$, sudden alarm calls of several red-fronted lemurs were heard in the study area known as CS7. Six individuals ( 2 adult females, 2 adult males, 1 juvenile male, 1 male infant) could be identified after approaching the group to within $10 \mathrm{~m}$. Three of them ( 2 adult females, 1 adult male) emitted "Woofs" and "Huvvs", vocalisations typically uttered during predator encounters (Fichtel and Kappeler 2002). The 5 individuals surrounded an approx. $2 \mathrm{~m}$ long Madagascar ground boa that was lying 
motionless on the ground. The lemurs sat at a height of 1-2 $\mathrm{m}$, each about $3 \mathrm{~m}$ away from the snake, wagging their tails vigorously, except the infant that kept a distance of $5 \mathrm{~m}$ during the entire event and did not display any vocalisations or tail-wagging. During the next $4 \mathrm{~min}$, one of the adult males approached the front end of the boa twice, getting as close as 1-2 $\mathrm{m}$. After $5 \mathrm{~min}$, he left the scene. During this time, one of the adult females also approached the snake up to within $2 \mathrm{~m}$. When the male left, the second female started to quickly circuit the boa for $4 \mathrm{~min}$, maintaining a distance of 2-3 $\mathrm{m}$. After $14 \mathrm{~min}$ of several approaches and continuous alarm calls by 3-5 individuals, the boa moved for the first time, heading slowly away. The remaining adult male approached the moving snake also within $2 \mathrm{~m}$; also at its front end. About 1 min later, the boa had moved $15 \mathrm{~m}$ away, and the lemurs left in the opposite direction, still uttering grunts continuously. Once the boa was out of sight, the mobbing stopped and the lemurs ' behaviour returned to baseline levels.

\section{Discussion}

The mobbing reaction of the group was strong and prolonged and included most of the group members. This is similar to the behaviour of a black lemur (Eulemur macaco macaco) group encountering a Madagascar boa at Ambato Massif, where the group mobbed the snake for $15-20 \mathrm{~min}$, and some individuals approached it as close as $1 \mathrm{~m}$ before finally leaving the location (Colquhoun 1993). It is also noteworthy that females and males mobbed and approached the snake in equal measure as observed in a number of other species (e.g., Tamura 1989; Ferrari and Ferrari 1990; Tello et al. 2002). The infant maintained a larger distance to the boa and did not engage in the mobbing displays, however. Similar infant behaviour has also been reported for other primates (e.g., Ferrari and Ferrari 1990) and might be due to a higher susceptibility to an attack due to smaller body size or a lack of innate experience regarding predator encounters and mobbing strategies (Curio et al. 1978; Fichtel, in press).

The strong mobbing reaction of the lemurs might be explained by the hunting strategy of the snake. Boas are ambush hunters that usually abandon an attack as soon as they have been detected (Montgomery and Rand 1978; Slip and Shine 1988). Therefore, it seems beneficial for prey animals to signal the ambush hunter quickly and distinctly that it has been detected. As boas do not pursue their prey after an unsuccessful attack, it is 
also unsurprising that the lemurs' behaviour returned to baseline levels of anxiety shortly after departing the site of the predator encounter. In contrast, groups of red-fronted lemurs showed increased vigilance behaviour for at least 30-60 min after encountering a fossa (Cryptoprocta ferox; pers. comm. Jean-Pierre Tolojanahary and pers. observation by LP), which is probably due to the higher agility and climbing abilities of the largest mammalian carnivore. Furthermore, fossas have been observed to hunt cooperatively and pursue prey up to 45 min (Lührs and Dammhahn 2009).

There are no quantitative data on predation rates of lemurs by snakes in Kirindy, only opportunistic observations (e.g., Schülke 2001; Eberle and Kappeler 2008) that are biased by several factors, however. The low observation rate of boa-lemur interactions could be due to the reptiles' nocturnal lifestyle (Raxworthy 2003), so that most of the attacks would occur at night when no or only few observers are working in the forest. Furthermore, boas at Kirindy are only active during the rainy season from January to April, when observations are often limited by dense foliage and frequent rainfalls. Five of the 6 individuals taking part in the mobbing were related ( 1 adult female and her 4 offspring from the last 4 years), while it is currently unknown whether the second adult male sired one of the two youngest group members. However, due to the small number of detailed observations of predator encounters it remains impossible to identify the ultimate causes of collective mobbing (kin defence/parental care, self-/group defence or cultural transmission of enemy recognition; Curio et al. 1978) in this species for the time being.

\section{Acknowledgements}

LWP was supported financially by the Deutsche Forschungsgemeinschaft (DFG; KA 1082/16-1, FuE). We thank field assistant Jean-Pierre Tolojanahary for sharing his longterm observation experiences with us. We are also grateful to Peter Kappeler, Claudia Fichtel and Moritz Rahlfs for constructive and helpful comments on earlier drafts of the manuscript. 


\section{GENERAL DISCUSSION}

In this thesis, I identified a number of terminological inconsistencies and conceptual shortcomings in studies on coordination and decision-making in animal groups (Chapter 1) as well as methodological concerns regarding the analysis of group ranging behaviour (Chapter 2) that hamper comparisons among studies and taxa. I will use the general discussion to briefly summarise how these problems could be reduced in future research. Subsequently, I will revisit the major findings from the empirical chapters, compare them to results from other studies, and suggest general patterns that are shared among different species. Finally, I will outline some approaches for future studies that could fill gaps in our current understanding of group coordination and collective decision-making.

\section{Terminology and concepts}

Research on coordination and decision-making has increased considerably throughout the last decade. The expeditious rise in the number of studies also resulted in several different definitions and concepts with only partial overlap (see Conradt and List 2009; Fischer and Zinner 2011 for discussions). Many of the recommendations to solve these problems made in Chapter 1 are straightforward. For instance, I proposed not to use the terms "combined decision" and "democratic/despotic decision" in future research to avoid ambiguity and anthropocentric connotations. A central but highly debated term in decision-making is leadership (Petit and Bon 2010). I recommended using the term leader for an individual that initiates a collective action and elicits following behaviour (without specifying the exact number/proportion of followers), which is similar to other definitions (Krause et al. 2000; King 2010). However, empirical studies may demand species- or context-specific solutions. For instance, King et al. (2008) defined the leader as the animal that arrived first at an artificial feeding patch because they were not able to observe the initiation process during morning departure due to poor light conditions (Guy Cowlishaw, pers. comm.). In any case, the definition used should be reported clearly in each study to facilitate comparison.

The same applies to the definitions of each phase of the coordination process, i.e. initiation, followership and termination, as well as the definition of successful group movements, the latter of which may demand a certain proportion of followers (e.g., Petit 
et al. 2009). Throughout Chapter 1, I argued that these definitions should be taxonspecific in order to collect meaningful data (see also Trillmich et al. 2004). For instance, the distance that an initiator has to cover to count as a starting attempt for a collective movement in a group of meerkats can be expected to differ from that in rhesus macaques, for example, because these species clearly differ in body size and group cohesion. In addition, I stressed the importance of analysing the entire process of collective actions from initiation to termination, in order to gain a complete picture, including potential changes of leadership or a second decision-making process during termination. Another conceptual recommendation relates to the differentiation between leadership and decision-making. Many studies equated consistent leadership with unshared consensus decision-making, and distributed leadership with (partially) shared decisions (King et al. 2008; Stueckle and Zinner 2008; Bonanni et al. 2010). However, a number of recent findings suggest that consensus decisions can be shared despite consistent leadership (Sueur and Petit 2008a; Bourjade et al. 2009). Therefore, characteristics of leadership should be considered separately from investigations of the mechanisms that mediate decision-making among followers (Bourjade and Sueur 2010).

A more general concern relates to the comparability of studies of free-ranging and captive groups (Chapter 1.2). Because in captive settings groups of animals can easily be video-taped and observed continuously, they offer the valuable opportunity to conduct detailed studies on behavioural mechanisms of initiation and followership (Sueur and Petit 2010; Jacobs et al. 2011). However, group decisions in captive groups lack ecological relevance because there is no predation risk or competition over food. Therefore, determinants of leadership in captivity can usually be expected to differ fundamentally from determinants under natural conditions (see also Barrett and Henzi 2005). A second concern raised in Chapter $\mathbf{1 . 2}$ is the relationship between theoretical and empirical studies. Computer models constitute a large part of recent coordination research. While these models are indispensable for our basic understanding of coordination mechanisms (Rands et al. 2003; Couzin et al. 2005), they are often based on a set of variables that can not be assessed in empirical studies. On the one hand, this is an inherent characteristic of models that try to identify basic patterns by simplification. On the other hand, theorists should also try to develop models that are closer to natural conditions and can be tested in empirical studies to control their reliability. In turn, empirical studies should include 
more experiments during which specific variables can be controlled and modified systematically.

Finally, Chapter 2 dealt with the impact of different methods and sampling regimes on spatial analyses of animal group movements. Using red-fronted lemur groups as a test case, I showed that home range estimates varied considerably, depending on the analytical method, sampling parameters and ecological seasonality as well as interactions between variables. As a consequence, I suggest choosing estimators depending on the study taxon and specific objectives, and providing results from different estimators in order to show the degree of variance in the data and facilitate direct comparisons among different taxa and sites.

\section{Coordination processes and decision-making in red-fronted lemurs}

The aim of the empirical work conducted for this thesis was to provide a comprehensive picture of coordination processes and the type of decision-making in free-ranging groups of red-fronted lemurs under varying natural and experimental conditions. Under natural conditions (Chapter 3), group cohesion was high and movements proceded in a coordinated fashion, with changes of leadership occurring in only $9 \%$ of observed movements. Individuals of different sex and age classes initiated movements successfully. However, leadership was highly skewed towards adult females, which elicited $78 \%$ of collective movements and were overtaken less often than male initiators. The prevalent explanation of female leadership relates to the compensation of additional energetic expenditure due to gestation and lactation (Erhart and Overdorff 1999; Fischhoff et al. 2007). However, patterns of leadership in seasonal breeding red-fronted lemurs were not affected by reproductive or ecological seasonality, which makes this explanation unlikely. Instead, I suggested that female philopatry, which results in strong genetic and affiliative network relations within groups, favoured joining behaviour, which in turn positively reinforced female leadership year-round. In order to test this hypothesis, I am currently analysing latencies and distances of joiners during group departures in relation to individual socio-genetic relations with the initiator of the movement.

Field observations also revealed that recruitment success was higher for adult initiators and during the dry season, but that it did not depend on the initiator's sex. The finding of adults leading more often than subadults matches those of numerous other 
studies and may be best explained by age-specific asymmetries in experience and knowledge and juvenile preference for safe spatial positions in the centre of the group (Boesch 1991a; Janson and van Schaik 1993; Boinski and Campbell 1995). Higher predation risk during the lean season (Janson and Goldsmith 1995) might account for higher numbers of followers. However, we did not adjust the operational definition of group movements used for red-fronted lemurs seasonally - including the radius around the terminator in which followers have to arrive. Therefore, higher recruitment success might simply have reflected higher overall group cohesion during the dry season. Finally, male leaders have been shown to have lower numbers of followers in female-dominated lemur species (Sauther ans Sussman 1993; Trillmich 2004). Therefore, the egalitarian structure of red-fronted lemur groups could explain the lack of sex-specific differences regarding recruitment success observed in this thesis.

In contrast to group movements between natural feeding and resting sites, coordination decreased considerably during the field experiment (Chapter 4). Adult females still initiated approx. 70\% of movements towards the platforms. However, the leader was overtaken by individuals of all sex and age classes in $67 \%$ and individual resource intake at the foraging platforms did not depend on departure order, sex or age but exclusively on arrival order. Furthermore, the group fissioned into 2-3 subgroups in $11 \%$ of platform visits which resulted in more individuals depleting the experimental resource simultaneously. I argued that the high value and predictability of the artificial resource did account for the changes in coordination processes between natural and experimental conditions. Thus, the benefits of "egoistically" exploiting the artificial resource must have outweighed a potential increase in predation risk through neglected coordination and temporarily low cohesion (Houston et al. 1993). In addition, individual aggressiveness at the platforms did not result in supplanting of conspecifics from the resource. Such equally distributed resource holding potential presumably reflects again the egalitarian social structure and high social tolerance of red-fronted lemurs (Kappeler 1991).

Due to random patterns of platform visits, I was unable to determine the decision type at departure using the experimental paradigm. In fact, red-fronted lemurs did not seem to rely on consensus decisions during departure towards the platforms but used alternative tactics (overtaking, group fission) to avoid consensus costs and maximise 
individual foraging benefits. However, it is possible to make some predictions regarding decision-making in red-fronted lemurs under natural conditions on the basis of findings of previous studies. For instance, groups of rather egalitarian Tonkean macaques showed equally shared decision-making involving a voting process and affiliative mimetism, whereas more despotic rhesus macaques exhibited consensus decisions that relied on a few old and dominant individuals (Sueur and Petit 2008a; Sueur et al. 2010). In addition, Jacobs et al. (2011) demonstrated that joining behaviour in brown lemurs depended on the actions of closely affiliated group members (selective mimetism). Due to the egalitarian structure of their groups and the close relatedness to brown lemurs, I also expect shared decision-making in red-fronted lemurs, presumably mediated by affiliative mimetism.

As mentioned in the general introduction, mechanisms of group coordination in terms of vocal communication were not part of this project but explored separately in a diploma thesis (Pflüger 2010) that I want to address briefly in order to provide a complete picture of group coordination in red-fronted lemurs. Preliminary results from Pflüger`s work suggest that red-fronted lemurs occasionally use a call combination ("hoo-gruntmeow") to initiate group movements (over large distances). However, systematic playback experiments to test this assumption have not yet been conducted.

\section{Conclusions}

Theoretical considerations presented in this thesis highlight the need for unambiguous terminology, clear research concepts, taxon-specific definitions and carefully designed theoretical models as well as field experiments in order to collect biologically meaningful and comparable data that expand our knowledge on coordination and decision-making in animal groups. While, naturally, any research field requires clear terminology and methodology, particular attention should be dedicated to this issue in studies on group coordination because they heavily rely on definitions of single terms - such as leadership, decision-making, consensus or self-organisation - that have been employed ambiguously in both, colloquial and scientific language for a long time (List and Conradt 2009; Chapter 1.3). Only if definitions and concepts are used consistently and reported clearly, it will be possible to integrate future studies in a coherent framework of animal group coordination. 
The empirical results of this thesis revealed that group coordination in freeranging red-fronted lemurs can yield highly flexible outcomes depending on a number of environmental parameters. Outcomes ranged from well-coordinated movements with stable leadership to "chaotic" movements including frequent change of leadership and splitting into subgroups. Determinants of group coordination included (i) sex and age, (ii) ecological seasonality, and (iii) high value/predictability of artificial foraging patches. Although females led group movements significantly more often than males, there was no sex-specific difference in recruitment success.

These results suggest a number of conclusions regarding the relationships among coordination, food-predation trade-offs and social structure in animal groups on a more general level. First, increased predation risk seems to promote higher cohesion/stronger followership during group movements. On the other hand, neglected coordination and temporal fissioning that can be expected to translate into costs of higher predation risk (Alexander 1974) can be outweighed by the selfish benefits of exploiting a high-value resource. The second conclusion relates to the impact of a species`social structure on group coordination. If a clear dominance hierarchy exists, usually dominant individuals lead and play a prominent role in the decision-making process (Sueur and Petit 2008a; Bonanni et al. 2010; Šárová et al. 2010). In a foraging experiment with baboons, subordinates even followed the dominant leader in most cases despite considerable consensus costs (King et al. 2008). In the absence of dominance, affiliative network relations seem to play the major role in governing group coordination (Sueur et al. 2010; Jacobs et al. 2011; Chapter 3). Furthermore, an egalitarian social structure presumably allows individuals to recruit group members successfully irrespective of sex (Chapter 3 ), and to use alternative behavioural tactics to consensus decisions that translate into individual foraging benefits irrespective of sex, age or social status (Chapter 4). 


\section{Outlook}

Research on group coordination and decision-making progressed immensely in the last decade. Nevertheless, the discussion of results from this thesis in relation to existing studies revealed considerable lacunae in our current knowledge. In particular, I encourage future research in the following areas:

1. Leadership is expected to be determined by a combination of different individual characteristics (King et al. 2009). These determinants can vary depending on the context of the collective action (King et al. 2008; Stueckle and Zinner 2008; Chapter 4). To unravel context-specific determinants, more empirical studies on group coordination of species differing in group composition and social structure during comparable situations, e.g., morning departures, movements between natural foraging patches or artificial feeding sites, are needed.

2. Recently, a number of studies demonstrated that behavioural variation or personality can be associated with leadership (Harcourt et al. 2009; Kurvers et al. 2009; Schuett and Dall 2009; see also Chapter 1). However, the impact of personality on group coordination is still poorly understood in most species and, therefore, represents a promising field for future research.

3. A central argument for leadership is motivation due to physiological requirements (Rands et al. 2003; Fischhoff et al. 2007; Chapter 3). Regular measures of body mass and field metabolic rates of different group members especially during energetically demanding periods like lactation (Scantlebury et al. 2002; Simmen et al. 2010) can help testing this assumption and should be included in upcoming coordination research.

4. Field experiments already proved highly valuable to analyse coordination mechanisms and decision types (Biro et al. 2006; Kerth et al. 2006; Chapter 4). An obvious approach for the future would be to experimentally vary group composition in terms of age, sex, relatedness, physiological condition or individual knowledge (e.g., using vibrating collars to inform particular individuals about a food resource) and to study the impact of these manipulations on coordination processes and mechanisms (Kerth 2010a). In this respect, mixed-species groups might offer a natural experiment because traits such as body size and knowledge 
of resource distribution often differ between the associated taxa (see Heymann 2011).

5. Finally, the need to coordinate is not restricted to collective movements. New and fascinating insights might also be gained by systematically studying coordination in other behavioural contexts, e.g., collective vigilance or joint mobbing of predators (Pays et al. 2007; Chapter 5). 


\section{SUMMARY}

In order to reap the benefits of group-living, gregarious animals have to maintain group cohesion. However, differences among group members in terms of sex, age or physiological constitution lead to different activity budgets and motivations, which, in turn, act as a centrifugal force on group cohesion. In order to stay cohesive despite inter-individual conflicts, groups must coordinate collective actions by making shared or unshared consensus decisions. In this context, single group members often adopt different behavioural roles, i.e. certain individuals emerge as leaders, others as followers. In the last decade, a number of studies explored single aspects of coordinated behaviour in the context of group movements, covering a wide range of taxa. Yet, comparisons among studies are still hampered by ambiguous terminology and inconsistent concepts. In addition, taxon- and condition-specific determinants of coordination processes and decision-making under natural conditions remain poorly understood.

I addressed both of these general issues in my thesis. First, I outlined why group movements emerged as the major paradigm of coordination research and why primates provide an excellent model taxon in this context, followed by a review of several aspects related to primate group movements. With this basis, I shifted the focus towards terminology, methodology and concepts used in (primate) coordination research and made suggestions to improve comparability among future studies. In particular, I encouraged the use of taxon-specific definitions of group movements and a clear differentiation between coordination research on the individual-level process (decision-making) and the group-level outcome (leadership).

In a second part of the thesis, I explored group movements in wild red-fronted lemurs (Eulemur rufifrons) with a comprehensive approach that included (i) analyses of spatio-temporal patterns of ranging behaviour using GPS collars, (ii) observations of coordination processes based on an operational definition in four groups across naturally variable socio-ecological contexts, and (iii) observations and video recordings of coordination and decision outcomes in one group during a field experiment aimed at manipulating the cost/benefit ratio of individual versus group-level decisions through the use of drinking platforms. Analyses of GPS ranging data revealed strong impacts of analytical method, sampling regime and seasonality on home range estimates. Under natural conditions, stable leadership by adult females prevailed year-round, presumably reinforced by close affiliative network relations among philopatric females. However, adult male initiators had the same recruitment success as females - supposedly a consequence of the species' egalitarian social structure. Recruitment success was higher during the lean season, possibly due to a higher predation risk. Adult females also initiated most movements during the experiment. However, leaders were overtaken in $67 \%$ of observed cases. Consequently, individual resource intake at the foraging platforms did not depend on sex, age or departure position but only on arrival order. Moreover, group fission occurred in $11 \%$ of cases, which resulted in more individuals drinking simultaneously but also temporarily low cohesion. The type of decisionmaking (shared or unshared) could not be determined because lemur visits at the platforms during the various patterns of baiting (monopolisable versus non-monopolisable resources) did not meet our predictions, but rather occurred randomly.

In conclusion, these results suggest that coordination processes are highly flexible, depending on environmental variations, such as seasonality and resource quality/predictability. Furthermore, adult recruitment success seems to be equally distributed among sexes, and group members may use alternative behavioural tactics to consensus decisions, such as overtaking or fissioning, in order to optimise individual foraging benefits irrespective of sex, age or social status. The findings in red-fronted lemurs might be representative of gregarious species characterised by egalitarian social relationships. 


\section{ZUSAMMENFASSUNG}

Gruppenlebende Tiere profitieren in vielfältiger Weise von der Vergesellschaftung mit anderen. Um die Gruppenkohäsion trotz individueller Interessenkonflikte zu gewährleisten, müssen die Aktivitäten einzelner Gruppenmitglieder durch gemeinsame Entscheidungen miteinander koordiniert werden. Diese Konsensentscheidungen können auf Grundlage der Interessen einiger weniger oder aller Gruppenmitglieder gefällt werden, wobei oftmals einzelne Individuen als Anführer fungieren, die den Rest der Gruppe rekrutieren. In den letzten Jahren wurden in zahlreichen theoretischen und empirischen Studien einzelne Aspekte der Gruppenkoordination am Beispiel von Gruppenbewegungen untersucht. Allerdings erschweren bislang uneinheitliche Begriffsdefinitionen und teils widersprüchliche Konzepte die Vergleichbarkeit einzelner Forschungsergebnisse. Auch die art- und umweltabhängigen Determinanten von Koordinationsprozessen und gemeinsamer Entscheidungsfindung unter natürlichen Bedingungen sind bislang wenig verstanden.

In der vorliegenden Dissertation habe ich beide der oben genannten Aspekte bearbeitet. Zunächst habe ich dargelegt, warum Bewegungen nicht-menschlicher Primaten ein ausgezeichnetes Modell darstellen, um Gruppenkoordination zu untersuchen, und zusammengefasst, was bislang in diesem Kontext erforscht wurde. Von dieser Datenbasis ausgehend, habe ich den Schwerpunkt auf die Bewertung von Begriffen, Methoden und Konzepten verlagert und aufgezeigt, wie die Vergleichbarkeit zwischen einzelnen Studien in Zukunft erleichtert werden kann. Insbesondere empfehle ich die Verwendung von artspezifischen Gruppenbewegungs-Definitionen und eine klare konzeptionelle Trennung von Studien, die Prozesse auf der Ebene des Individuums (Entscheidungsfindung) bzw. auf der Ebene der Gruppe (Anführerschaft) behandeln.

Im zweiten Teil dieser Arbeit habe ich Gruppenbewegungen bei Rotstirnmakis (Eulemur rufifrons) untersucht. Der Forschungsansatz umfasste (i) die Analyse zeitlich-räumlicher Bewegungsmuster mit Hilfe von GPS-Halsbändern, (ii) die Beobachtung von Koordinationsprozessen in unterschiedlichen sozio-ökologischen Kontexten sowie (iii) Beobachtungen und Videoaufnahmen von Koordinationsprozessen und Konsequenzen von Gruppenentscheidungen im Rahmen eines Feldexperiments mit manipuliertem Konfliktpotential. Die Analyse der GPS-Daten zeigte starke Einflüsse der Berechnungsmethoden sowie der klimatischen Saisonalität auf die Streifgebietsgröße. Unter natürlichen Bedingungen führten ganzjährig überwiegend adulte Weibchen die Gruppen an, vermutlich als Folge enger affiliativer Netzwerk-Beziehungen durch weibliche Philopatrie. Allerdings hatten männliche Initiatoren denselben Rekrutierungserfolg wie weibliche, was als Konsequenz der egalitären Sozialstruktur bei Rotstirnmakis verstanden werden kann. Der Rekrutierungserfolg war generell während der Trockenzeit höher, womöglich eine Folge von erhöhtem Prädationsrisiko. Adulte Weibchen initiierten auch die meisten Bewegungen während des Experiments. Allerdings wurden die Anführer in $67 \%$ der Fälle überholt. Dementsprechend bestimmten weder die Startreihenfolge noch Geschlecht oder Alter die Ressourcenaufnahme auf den Plattformen, sondern allein die Ankunftsreihenfolge. Zudem teilte sich die Gruppe in $11 \%$ der Fälle in Subgruppen auf, wodurch mehr Individuen gleichzeitig von der experimentellen Ressource profitierten. Wie viele Individuen jeweils an den Entscheidungen beteiligt waren, die den Besuchen von unterschiedlich bestückten Plattformen vorausgingen, ließ sich nicht feststellen, da die Besuchsmuster zufällig waren.

Zusammengefasst weisen die Ergebnisse dieser Arbeit darauf hin, dass Koordinationsprozesse innerhalb einer Art in Abhängigkeit von den Umweltbedingungen stark variieren können (ökologische Saisonalität, Vorhersagbarkeit/Qualität der Ressource). Zudem scheint bei Arten mit egalitären Sozialbeziehungen der Rekrutierungserfolg geschlechtsunabhängig zu sein, und Gruppenmitglieder können unabhängig von Alter, Geschlecht oder sozialem Rang alternative Verhaltenstaktiken zu Konsensentscheidungen wählen (Überholen, temporäre Gruppenteilung), um die individuelle Ressourcenaufnahme zu optimieren. 


\section{REFERENCES}

Akaike H (1974) A new look at the statistical model identification. IEEE Trans Autom Con 19:716723

Alberts SC, Altmann J, Wilson ML (1996) Mate guarding constrains foraging activity of male baboons. Anim Behav 51:1269-1277

Alexander RD (1974) The evolution of social behavior. Annu Rev Ecol Syst 5:325-383

Alexander RD (1987) The biology of moral systems. Aldine, London

Altizer S, Nunn CL, Thrall PH, Gittleman JL, Antonovics J, Cunningham AA, Dobson AP, Ezenwa V, Jones KE, Pedersen AB, Poss M, Pulliam JRC (2003) Social organization and parasite risk in mammals: Integrating theory and empirical studies. Annu Rev Ecol Evol Syst 34:517-547

Altmann J (1974) Observational study of behavior: sampling methods. Behaviour 49:227-267

Altmann J (1980) Baboon mothers and infants. Harvard Univ Press, Cambridge

Altmann SA (1979) Baboon progressions: order or chaos? A study of one dimensional group geometry. Anim Behav 27:46-80

Anderson C (1981) Subtrooping in a chacma baboon (Papio ursinus) population. Primates 23:445458

Arthur SM, Schwartz CC (1999) Effects of sample size on accuracy and precision of brown bear home range models. Ursus 11:139-148

Aureli F, Cords M, van Schaik CP (2002) Conflict resolution following aggression in gregarious animals: a predictive framework. Anim Behav 64:325-343

Baker, M. (2001). Variation in ranging patterns and habitat use by capuchin monkeys (Cebus capucinus) in a dry tropical forest. Am J Primatol 54:95

Barrett L, Henzi P (2005) The social nature of primate cognition. Proc R Soc Lond B 272:1865-1875

Barthold J, Fichtel C, Kappeler PM (2009) What is it going to be? Pattern and potential function of natal coat change in sexually dichromatic redfronted lemurs (Eulemur fulvus rufus). Am J Primatol 138:1-10

Bates DM, Sarkar D (2007) Ime4: linear mixed-effects models using S4 classes. In: R package version 0.9975-12, URL http://CRAN.R-project.org/

Beauchamp G (2000) Individual differences in activity and exploration influence leadership in pairs of foraging zebra finches. Behaviour 137:301-314

Benadi G, Fichtel C, Kappeler P (2008) Intergroup relations and home range use in Verreaux's sifaka (Propithecus verreauxi). Am J Primatol 70:956-965

Bercovitch FB (1983) Time budgets and consortships in olive baboons (Papio anubis). Folia Primatol 41:180-190

Bertram BCR (1978) Living in groups: predators and prey. In: Krebs JR, Davies JB (eds) Behavioural ecology, 3rd edn. Blackwell Scientific, Oxford, pp 64-96

Biro D, Sumpter DJT, Meade J, Guilford T (2006) From compromise to leadership in homing pigeons. Curr Biol 16:2123-2128

Black JM (1988) Preflight signaling in swans - a mechanism for group cohesion and flock formation. Ethology 79:143-157

Blundell GM, Maier JAK, Debevec EM (2001) Linear home ranges: effects of smoothing, sample size, and autocorrelation on kernel estimates. Ecol Monogr 71:469-489

Börger L, Franconi N, de Michele G, Gantz A, Meschi F, Manica A, Lovari S, Coulson T (2006) Effects of sampling regime on the mean and variance of home range size estimates. J Anim Ecol 75:1393-1405

Boesch C (1991a) The effects of leopard predation on grouping patterns in forest chimpanzees. Behaviour 117:220-242

Boesch C (1991b) Symbolic communication in wild chimpanzees? Hum Evol 6:81-90

Boinski S (1988) Sex differences in the foraging behavior of squirrel monkeys in a seasonal habitat. Behav Ecol Sociobiol 23:177-186 
Boinski S (1991) The coordination of spatial position: a field study of the vocal behaviour of adult female squirrel monkeys. Anim Behav 41:89-102

Boinski S (1993) Vocal coordination of group movement among white-faced capuchin monkeys, Cebus capucinus. Am J Primatol 30:85-100

Boinski S (2000) Social manipulation within and between troops mediates primate group movement. In: Boinski S, Garber PA (eds) On the move: how and why animals travel in groups. Univ of Chicago Press, Chicago, pp 421-469

Boinski S, Campbell AF (1995) Use of trill vocalisations to coordinate troop movement among white-faced capuchins: a second field test. Behaviour 132:875-901

Boinski S, Cropp S (1999) Disparate data sets resolve squirrel monkey (Saimiri) taxonomy: Implications for behavioral ecology and biomedical usage. Int J Primatol 20:237-256

Boinski S, Garber PA (eds) (2000) On the move: How and why animals travel in groups. Univ of Chicago Press, Chicago

Boinski S, Moraes E, Kleiman D, Dietz J, Baker A (1994) Intra-group vocal behavior in wild golden lion tamarins, Leontopithecus rosalia: Honest communication of individual activity. Behaviour 130:53-76

Bonanni R, Cafazzo S, Valsecchi P, Natoli E (2010) Effect of affiliative and agonistic relationships on leadership behaviour in free-ranging dogs. Anim Behav 79:981-991

Bourjade M, Sueur C (2010) Shared or unshared consensus to move collectively? Towards methodological concerns. Behav Process 84:648-652

Bourjade M, Thierry B, Maumy M, Petit O (2009) Decision-making in Przewalski horses (Equus ferus przewalskii) is driven by the ecological contexts of collective movements. Ethology 115:321-330

Boulanger JG, White GC (1990) A comparison of home-range estimators using Monte Carlo simulation. J Wildl Manage 54:310-315

Bousquet CAH, Sumpter DJT, Manser MB (2010) Moving calls: a vocal mechanism underlying quorum decisions in cohesive groups. Proc R Soc Lond B, doi:10.1098/rspb.2010.1739

Bowman JL, Kochanny CO, Demarais S, Leopold BD (2000) Evaluation of a GPS collar for whitetailed deer. Wildl Soc B 28:141-145

Boyle SA, Lourenço WC, da Silva LR, Smith AT (2009) Home range estimates vary with sample size and method. Folia Primatol 80:33-42

Braune P, Schmidt S, Zimmermann E (2005) Spacing and group coordination in a nocturnal primate, the golden brown mouse lemur (Microcebus ravelobensis): the role of olfactory and acoustic signals. Behav Ecol Sociobiol 58:587-596

Brockman DK (1999) Reproductive behaviour of female Propithecus verreauxi at Beza Mahafaly, Madagascar. Int J Primatol 20:375-398

Brockman DK, van Schaik CP (2004) Primate seasonality: Implications for human evolution. Cambridge Univ Press, Cambridge

Brown JL (1987) Helping and communal breeding in birds: Ecology and evolution. Princeton Univ Press, Princeton

Bulger JB (1993) Dominance rank and access to estrous females in male savanna baboons. Behaviour 127:67-103

Burgman MA, Fox JC (2003) Bias in species range estimates from minimum convex polygons: implications for conservation and options for improved planning. Anim Conserv 6:19-28

Burney DA (2002) Sifaka predation by a large boa. Folia Primatol 73:144-145

Byrne RW (1981) Distance vocalization of Guinea baboons (Papio papio): an analysis of function. Behaviour 78:283-312

Byrne RW (2000) How monkeys find their way: leadership, coordination, and cognitive maps of African baboons. In: Boinski S, Garber P (eds) On the move. Univ of Chicago Press, Chicago, pp 491-518

Cagnacci F, Boitani L, Powell RA, Boyce MS (2010) Animal ecology meets GPS-based radiotelemetry: a perfect storm of opportunities and challenges. Phil Trans R Soc Lond B 365:2157-2162 
Camazine S, Deneubourg JL, Franks NR, Sneyd J, Theraulaz G., Bonabeau E (2001) Selforganization in biological systems. Princeton Univ Press, Princeton

Caro T (1998) The significance of behavioral ecology for conservation biology. In: Caro T (ed) Behavioral Ecology and conservation biology. Oxford Univ Press, New York, pp 3-26

Caro T (2005) Antipredator defenses in birds and mammals. Univ of Chicago Press, Chicago

Cavalcanti SMC, Gese EM (2009) Spatial ecology and social interactions of jaguars (Panthera onca) in the southern Pantanal. Braz J Mammal 90:935-945

Chapman CA (1990) Association patterns of spider monkeys: the influence of ecology and sex on social organization. Behav Ecol Sociobiol 26:409-414

Chapman CA, Wrangham R, Chapman L (1995) Ecological constraints on group size: an analysis of spider monkey and chimpanzee subgroups. Behav Ecol Sociobiol 36:59-70

Cheney DL (1987) Interactions and relationships between groups. In: Smuts BB, Cheney DL, Seyfarth RM, Wrangham RW, Struhsaker TT (eds) Primate societies. Univ of Chicago Press, Chicago, pp 267-281

Cheney DL (1992) Intragroup cohesion and intergroup hostility: the relation between grooming distributions and intergroup competition among female primates. Behav Ecol 3:334-345

Cheney DL, Seyfarth RM (2007) Baboon metaphysics: the evolution of a social mind. Univ of Chicago Press, Chicago

Clutton-Brock TH (1975) Ranging behavior of red colobus (Colobus badius tephrosceles) in the Gombe National Park. Folia Primatol, 19:368-379

Colquhoun IC (1993) The socioecology of Eulemur macaco: A preliminary report. In: Kappeler PM, Ganzhorn JU (eds) Lemur social systems and their ecological basis. Plenum Press, New York, pp 11-23

Conradt L (2011) When it pays to share decisions. Nature 471:40-41

Conradt L, Roper TJ (2003) Group decision making in animals. Nature 421:155-158

Conradt L, Roper TJ (2005) Consensus decision making in animals. Trends Ecol Evol 20:449-456

Conradt L, Roper TJ (2007) Democracy in animals: the evolution of shared group decisions. Proc R Soc Lond B 274:2317-2326

Conradt L, List C (2009) Introduction. Group decisions in humans and animals: a survey. Proc R Soc Lond B 364:719-742

Conradt L, Krause J, Couzin ID, Roper TJ (2009) "Leading according to need" in self-organizing groups. Am Nat 173:304-312

Cornelius C, Boos M (2003) Enhancing mutual understanding in synchronous computer-mediated communication by training: trade-offs in judgmental tasks. Commun Res 30:147-177

Couzin I, Krause J (2003) Self-organization and collective behavior in vertebrates. Adv Stud Behav 32:1-7

Couzin ID, Krause J, James R, Ruxton GD, Franks NR (2002) Collective memory and spatial sorting in animal groups. J Theor Biol 218:1-11

Couzin ID, Krause J, Franks NR, Levin SA (2005) Effective leadership and decision-making in animal groups on the move. Nature 433:513-516

Curio E, Ernst U, Vieth W (1978) Cultural transmission of enemy recognition: one function of mobbing. Science 202:899-901

Deneubourg JL, Goss S (1989) Collective patterns and decision-making. Ethol Ecol Evol1:295-311

Di Orio AP, Callas R, Schaefer RJ (2003) Performance of two GPS telemetry collars under different habitat conditions. Wildl Soc B 31:372-379

Dominy NJ, Duncan B (2001) GPS and GIS methods in an African rain forest: applications to tropical ecology and conservation. Conserv Ecol 5, http://www.consecol.org/vol5/iss2/art6/

Doran D (1997) Influence of seasonality on activity patterns, feeding behavior, ranging, and grouping patterns in Taï chimpanzees. Int J Primatol 18:183-206

Dumont B, Boissy A, Achard C, Sibbald AM, Erhard HW (2005) Consistency of animal order in spontaneous group movements allows the measurement of leadership in a group of grazing heifers. Appl Anim Behav Sci 95:55-66 
Dunbar RIM (1988) Primate social systems. Cornell Univ Press, Ithaca

Dunbar RIM, Dunbar EP (1975) Social dynamics of gelada baboons. Contrib Primatol 6:1-157

Dunbar RIM, Dunbar EP (1988) Maternal time budgets of gelada baboons. Anim Behav 36:970-980

Dunbar RIM, Shultz S (2007) Evolution in the social brain. Science 317:1344-1347

Dyer JRG, Ioannou CC, Morrell L, Croft DP, Couzin ID, Waters DA, Krause J (2008) Consensus decision making in human crowds. Anim Behav 75:461-470

Dyer JRG, Johansson A, Helbing D, Couzin ID, Krause J (2009) Leadership, consensus decision making and collective behavior in humans. Phil Trans R Soc Lond B 364:781-789

Eberle M, Kappeler PM (2008) Mutualism, reciprocity, or kin selection? Cooperative rescue of a conspecific from a boa in a nocturnal solitary forager the gray mouse lemur. Am J Primatol 70:410-414

Eisenberg JF (1981) The mammalian radiations. Univ of Chicago Press, Chicago

Erhart EM, Overdorff DJ (1998) Group leadership and feeding priority in wild Propithecus diadema edwardsi and Eulemur fulvus rufus. Am J Primatol 45:178-179

Erhart EM, Overdorff DJ (1999) Female coordination of group travel in wild Propithecus and Eulemur. Int J Primatol 20:927-941

Erwin N, Erwin J (1976) Social density and aggression in captive groups of pigtail monkeys (Macaca nemestrina). Appl Anim Ethol 2:265-269

Faraway JJ (2005) Linear models with R. Chapman \& Hall, London

Faraway JJ (2006) Extending the linear model with R. Chapman \& Hall, London

Fashing PJ (2001) Male and female strategies during intergroup encounters in guerezas (Colobus guereza): evidence for resource defense mediated through males and a comparison with other primates. Behav Ecol Sociobiol 50:219-230

Feist JD, McCullough DR (1976) Behaviour patterns and communication in feral horses. Z Tierpsychol 41:337-371

Ferrari SF, Ferrari MAL (1990) Predator avoidance behaviour in the buffy-headed marmoset, Callithrix flaviceps. Primates 31:323-338

Fichtel C (in press) Predation on primates. In: Mitani JC, Call J, Kappeler PM, Palombit R, Silk J (eds) The evolution of primate societies. Univ of Chicago Press, Chicago

Fichtel C, Kappeler PM (2002) Anti-predator behaviour of group-living Malagasy primates: mixed evidence for a referential alarm call system. Behav Ecol Sociobiol 51:262-275

Fichtel C, Pyritz L, Kappeler PM (2011) Coordination of group movements in non-human primates. In: Boos M, Kolbe M, Ellwart S, Kappeler PM (eds) Coordination in human and non-human primate groups. Springer, Heidelberg, pp 37-56

Fischer J, Zinner D (2011) Communicative and cognitive underpinnings of animal group movement. In: Boos M, Kolbe M, Kappeler PM, Ellwart T (eds) Coordination in human and primate groups. Springer, Heidelberg, pp 229-244

Fischhoff IR, Sundaresan SR, Cordingley J, Larkin HM, Sellier M-J, Rubenstein DI (2007) Social relationships and reproductive state influence leadership roles in movements of plains zebra, Equus burchellii. Anim Behav 73:825-831

Fortin MJ, Dale MRT (2009) Spatial autocorrelation in ecological studies: a legacy of solutions and myths. Geogr Anal 41:392-397

Franks NR, Pratt SC, Mallon EB, Britton NF, Sumpter DJT (2002) Information flow, opinion polling and collective intelligence in house-hunting social insects. Phil Trans R Soc B 357:15671583

Franks NR, Dornhaus A, Fitzsimmons JP, Stevens M (2003) Speed versus accuracy in collective decision-making. Proc R Soc Lond B: 270:2457-2463

Furrer RD, Kyabulima S, Willems EP, Cant MA, Manser MB (2011) Location and group size influence decisions in simulated intergroup encounters in banded mongooses. Behav Ecol 22:493-500

Ganzhorn JU, Sorg JP (1996) Ecology and economy of a tropical dry forest in Madagascar. Prim Rep 46-1, Göttingen, Goltze 
Ganzhorn JU, Wright P, Ratsimbazafy J (1999) Primate communities: Madagascar. In: Fleagle JG, Janson $\mathrm{CH}$ (eds) Primate communities. Cambridge Univ Press, Cambridge, pp 75-89

Girard I, Ouellet JP, Courtois R, Dussault C, Breton L (2002) Effects of sampling effort based on GPS telemetry on home-range size estimations. J Wildl Manage 66:1290-1300

Gitzen RA, Millspaugh JJ (2003) Comparison of least-squares cross-validation bandwidth options for kernel home-range estimation. Wildl Soc B 31:823-831

Goldizen AW (1987b) Tamarins and marmosets: communal care of offspring. In: Smuts BB, Cheney DL, Seyfarth RM, Wrangham RW, Struhsaker TT (eds) Primate societies. Univ of Chicago Press, Chicago, pp 33-43

Gompper ME (1996) Sociality and asociality in white-nosed coatis (Nasua narica): foraging costs and benefits. Behav Ecol 7:254-265

Goodall J (1968) The behavior of free-living chimpanzees in the Gombe Stream Reserve. Anim Behav Monogr 1:161-311

Grüter CC, Li D, Ren B, Wei F (2009) Choice of analytical method can have dramatic effects on primate home range estimates. Primates 50:81-84

Harcourt JL, Ang TZ, Sweetman G, Johnstone RA, Manica A (2009) Social feedback and the emergence of leaders and followers. Curr Biol 19:248-252

Harris S, Cresswell WJ, Forde PG, Trewhella WJ, Woollard T, Wray S (1990) Home-range analysis using radio-tracking data - a review of problems and techniques particularly as applied to the study of mammals. Mammal Rev 20:97-123

Harris TR, Chapman CA (2007) Variation in diet and ranging of black and white colobus monkeys in Kibale National Park, Uganda. Primates 48:208-221

Hawkins CE, Racey PA (2008) Food habits of an endangered carnivore, Cryptoprocta ferox, in the dry deciduous forests of Western Madagascar. J Mammal 89:64-74

Hemelrijk CK (2002) Understanding social behavior with the help of complexity science. Ethology 108:655-671

Hemson G, Johnson P, South A, Kenward R, Ripley R, MacDonald D (2005) Are kernels the mustard? Data from global positioning system (GPS) collars suggests problems for kernel home-range analyses with least-squares cross-validation. J Anim Ecol 74:455-463

Heymann EW (2011) Coordination in primate mixed-species groups. In: Boos M, Kolbe M, Kappeler PM, Ellwart T (eds) Coordination in human and primate groups. Springer, Heidelberg, pp 263-281

Horvath JE, Weisrock DW, Embry SL, Fiorentino I, Balhoff JP, Kappeler PM, Wray GA, Willard HF, Yoder AD (2008) Development and application of a phylogenomic toolkit: resolving the evolutionary history of Madagascar's lemurs. Genome Res 18:489-499

Hoset KS, Le Galliard JF, Gundersen G, Steen H (2008) Home range size and overlap in female root voles: effects of season and density. Behav Ecol 19:139-145

Houston Al, McNamara JM, Hutchinson JCM (1993) General results concerning the trade-off between gaining energy and avoiding predation. Phil Trans R Soc B 341:375-397

Ingmanson EJ (1996) Tool using behavior in wild Pan paniscus: social and ecological considerations. In: Russon AE, Bard K, Taylor S (eds) Reaching into thought: the minds of the great apes. Cambridge Univ Press, Cambridge, pp 190-210

Irwin MT (2008) Diademed sifaka (Propithecus diadema) ranging and habitat use in continuous and fragmented forest: higher density but lower viability in fragments? Biotropica 40:231240

Isbell LA, Cheney DL, Seyfarth RM (1990) Costs and benefits of home range shifts among vervet monkeys (Cercopithecus aethiops) in Amboseli National Park, Kenya. Behav Ecol Sociobiol 27:351-358

Itani J (1963) Vocal communication of the wild Japanese monkeys. Primates 4:11-66

Jacobs A (2010) Group cohesiveness during collective movements: Travelling apart together. Behav Pocess 84:678-680

Jacobs A, Maumy M, Petit O (2008) The influence of social organisation on leadership in brown lemurs (Eulemur fulvus fulvus) in a controlled environment. Behav Process 79:111-113 
Jacobs A., Sueur C., Deneubourg JL, Petit O (2011) Social network influences decision-making during collective movements in brown lemurs (Eulemur fulvus fulvus). Int J Primatol, doi:10.1007/s10764-011-9497-8

Janson CH (1998) Experimental evidence for spatial memory in foraging wild capuchin monkeys, Cebus apella. Anim Behav 55:1229-1243

Janson CH (2007) Experimental evidence for route integration and strategic planning in wild capuchin monkeys. Anim Cogn 10:341-356

Janson CH, van Schaik CP (1993) Ecological risk aversion in juvenile primates: slow and steady wins the race. In: Pereira ME, Fairbanks LA (eds) Juvenile primates life history, development and behavior. Oxford Univ Press, Oxford, pp 57-74

Janson CH, Goldsmith ML (1995) Predicting group size in primates: foraging costs and predation risk. Behav Ecol 6:326-336

Janson CH, Di Bitteti MS (1997) Experimental analysis of food detection in capuchin monkeys: effects of distance, travel speed, and resource size. Behav Ecol Sociobiol 41:17-24

Janzen D (1970) Altruism by coatis in the face of predation by boa constrictor. J Mammal 51:387389

Jouventin P (1975) Observations sur la socioecologie du mandrill [in French]. Terre Vie 29:493-532

Kappeler PM (1991) Patterns of sexual dimorphism in body weight among prosimian primates. Folia Primatol 57:132-146

Kappeler PM (1999) Convergence and divergence in primate social systems. In: Fleagle JG, Janson $\mathrm{CH}$, Reed K (eds) Primate communities. Cambridge University Press, Cambridge, pp 158170

Kappeler PM (2000) Grouping and movement patterns in Malagasy primates. In: Boinski S, Garber PA (eds) On the move: how and why animals travel in groups. Univ of Chicago Press, Chicago, pp 470-490

Kappeler PM (2011) Primatological approaches to the study of group coordination. In: Boos M, Kolbe M, Kappeler PM, Ellwart T (eds) Coordination in human and primate groups. Springer, Heidelberg, pp 223-228

Kappeler P, Port M (2008) Mutual tolerance or reproductive competition? Patterns of reproductive skew among male redfronted lemurs (Eulemur fulvus rufus). Behav Ecol Sociobiol 62:1477-1488

Kappeler PM, Schäffler L (2008) The lemur syndrome unresolved: Extreme male reproductive skew in sifakas (Propithecus verreauxi), a sexually monomorphic primate with female dominance. Behav Ecol Sociobiol 62:1007-1015

Kar Gupta K (2007) Socioecology and conservation of the slender loris (Loris tardigradus) in Southern India. Tempe, Arizona State Univ, PhD dissertation

Kerth G (2010a) Group decision-making in animal societies. In: Kappeler PM (ed) Behavior: Evolution \& Mechanisms. Springer, Heidelberg, pp 241-265

Kerth $\mathrm{G}(2010 \mathrm{~b})$ Group decision-making in fission-fusion societies. Behav Process 84:662-663

Kerth G, Ebert C, Schmidtke C (2006) Group decision making in fission-fusion societies: evidence from two field-experiments in Bechstein's bats. Proc R Soc Lond B 273:2785-2790

King AJ (2010) Follow me! I'm a leader if you do; I'm a failed initiator if you don't?. Behav Process 84:671-674

King AJ, Cowlishaw G (2009) Leaders, followers and group decision-making. Commun Integr Biol 2:147-150

King AJ, Sueur C (in press) Where next? Group coordination and collective decision-making by primates. Int J Primatol 32 (special issue)

King AJ, Douglas CMS, Huchard E, Isaac NJB, Cowlishaw G (2008) Dominance and affiliation mediate despotism in a social primate. Curr Biol, 18, 1833-1838

King AJ, Johnson DDP, Van Vugt M (2009) The origins and evolution of leadership. Curr Biol 19:911-916

Koenig A (2002) Competition for resources and its behavioral consequences among female primates. Int J Primatol 23:759-783 
Krause J, Ruxton GD (2002) Living in groups. Oxford University Press, New York

Krause J, Reeves P, Hoare D (1998) Positioning behavior in roach shoals: the role of body length and nutritional state. Behaviour 135:1031-1039

Krause J, Hoare D, Krause S, Hemelrijk CK, Rubenstein DI (2000) Leadership in fish shoals. Fish Fish 1:82-89

Kudo $\mathrm{H}$ (1987) The study of vocal communication of wild mandrills in Cameroon in relation to their social structure. Primates 28:289-308

Kümmeth F, Heidrich WA (2007) System specification for BaseStation and GPS-RF-tags, Version 0.2. Grünwald, e-obs GmbH, http://www.e-obs.de/index.html

Kummer H (1968) Social organisation of hamadryas baboons. Univ of Chicago Press, Chicago

Kummer H (1995) In quest of the sacred baboon: a scientist's journey. Princeton Univ Press, Princeton

Kurvers RHJM, Eijkelenkamp B, van Oers K, van Lith B, van Wieren SE, Ydenberg RC, Prins HHT (2009) Personality differences explain leadership in barnacle geese. Anim Behav 78:447453

Leblond C, Reebs SG (2006) Individual leadership and boldness in shoals of golden shiners (Notemigonus crysoleucas). Behaviour 143:1263-1280

Leca JB, Gunst N, Thierry B, Petit O (2003) Distributed leadership in semi-free ranging white-faced capuchin monkeys. Anim Behav 66:1045-1052

Lewis JS, Wartzok D, Heithaus MR (2011) Highly dynamic fission-fusion species can exhibit leadership when travelling. Behav Ecol Sociobiol 65:1061-1069

Li B, Chen C, Ji W, Ren B (2000) Seasonal home range changes of the Sichuan snub-nosed monkey (Rhinopithecus roxellana) in the Qinling Mountains of China. Folia Primatol 71:375-386

Lindburg DG (1987) Seasonality of reproduction in primates. In: Mitchell G, Erwin J (eds) Comparative primate biology, vol. $2 \mathrm{~b}$ : behavior, cognition and motivation. Alan $\mathrm{R}$ Liss, New York. pp 167-218

Lührs ML, Dammhahn M (2009) An unusual case of cooperative hunting in a solitary carnivore. Journal of Ethology, doi:10.1007/s10164-009-0190-8

Markham AC, Altmann J (2008) Remote monitoring of primates using automated GPS technology in open habitats. Am J Primatol 70:1-5

Marler P (1965) Communication in monkeys and apes. In: DeVore I (ed) Primate behavior. Holt Rinehart Winston, New York, pp 544-584

Matsumura S (1999) The evolution of "egalitarian" and "despotic" social systems among macaques. Primates 40:23-31

Maynard Smith J, Szathmáry E (1995) The major transitions in evolution. WHFreeman/Spektrum, Oxford

Mech LD (1970) The wolf: The ecology and behavior of an endangered species. Natural History Press, New York

Mehlman PT (1996) Branch shaking and related displays in wild Barbary macaques. In: Fa JE, Lindburg DG (eds) Evolution and ecology of macaque societies. Cambridge Univ Press, Cambridge, pp 503-526

Menzel CR (1993) Coordination and conflict in Callicebus social groups. In: Mason WA, Mendoza SP (eds) Primate social conflict. State Univ of New York Press, Albany, pp 253-290

Meunier H, Leca JB, Deneubourg JL, Petit O (2006) Group movement decisions in capuchin monkeys: the utility of an experimental study and a mathematical model to explore the relationship between individual and collective behaviours. Behaviour 143:1511-27

Meunier H, Deneubourg JL, Petit O (2008) How many for dinner? Recruitment and monitoring by glances in capuchins. Primates 49:26-31

Milton K (1980) The foraging strategy of howler monkeys: a study in primate economics. Columbia Univ Press, New York

Mitani JC, Call J, Kappeler PM, Palombit R, Silk J (in press) The evolution of primate societies. Univ of Chicago Press, Chicago 
Mitchell CL, Boinski S, van Schaik CP (1991) Competitive regimes and females bonding in two species of squirrel monkey (Saimiri oerstedi and S. sciureus). Behav Ecol Sociobiol 28:5560

Mittermeier R, Ganzhorn J, Konstant W, Glander K, Tattersall I, Groves C, Rylands A, Hapke A, Ratsimbazafy J, Mayor M, Louis E, Rumpler Y, Schwitzer C, Rasoloarison R (2008). Lemur diversity in Madagascar. Int J Primatol 29:1607-1656

Montgomery GG, Rand AS (1978) Movements, body temperature and hunting strategy of a Boa constrictor. Copeia 3:532-533

Moody AL, Houston Al, McNamara JM (1996) Ideal free distributions under predation risk. Behav Ecol Sociobiol 38:131-143

Mundry R, Nunn CL (2009) Stepwise model fitting and statistical inference: turning noise into signal pollution. Am Nat 173:119-123

Neville MK (1968) Ecology and activity of Himalayan foothill rhesus monkeys (Macaca mulatta). Ecology 49:110-123

Norton GW (1986) Leadership decision processes of group movement in yellow baboons. In: Else JG, Lee PC (eds) Primate ecology and conservation. Cambridge Univ Press, Cambridge, pp 145-156

Noser R, Byrne RW (2007a) Travel routes and planning of visits to out-of-sight resources in wild chacma baboons (Papio ursinus). Anim Behav 73:257-266

Noser R, Byrne RW (2007b) Mental maps in chacma baboons (Papio ursinus): Using inter-group encounters as a natural experiment. Anim Cogn 10:331-340

Nunn CL, Pereira ME (2000) Group histories and offspring sex ratios in ringtaild lemurs (Lemur catta). Behav Ecol Sociobiol 48:18-28

Oates JF (1977) The guereza and its food. In: Clutton-Brock TH (ed) Primate ecology. Academic, London, pp 276-321

Obbard ME, Pond BA, Perera A (1998) Preliminary evaluation of GPS collars for analysis of habitat use and activity patterns of black bears. Ursus 10:209-217

Oftedal OT (1985) Pregnancy and lactation. In: Hudson RJ, White RG (eds) The bioenergetics of wild herbivores. CRC Press, Boca Raton, pp 215-238

Osborne PE, Glew L (2011) Geographical information systems and remote sensing. In: Setchell JM, Curtis DJ (eds) Field and laboratory methods in primatology. Cambridge Univ Press, Cambridge, pp 69-89

Ostner J, Kappeler PM (1999) Central males instead of multiple pairs in redfronted lemurs, Eulemur fulvus rufus (Primates, Lemuridae)? Anim Behav 58:1069-1078

Ostner J, Kappeler PM (2004) Male life history and the unusual sex ratios of redfronted lemurs (Eulemur fulvus rufus) groups. Anim Behav 67:249-259

Overdorff DJ (1993a) Similarities, differences, and seasonal patterns in diets of Eulemur rubriventer and Eulemur fulvus rufus in the Ranomafana National Park, Madagascar. Int J Primatol 14:721-753

Overdorff DJ (1993b) Ecological and reproductive correlates to range use in red-bellied lemurs (Eulemur rubriventer) and rufous lemurs (Eulemur fulvus rufus). In: Kappeler PM, Ganzhorn JU (eds) Lemur social systems and their ecological basis. Plenum, New York, pp 167-178

Overdorff DJ (1996) Ecological correlates to activity and habitat use of two prosimian primates Eulemur rubriventer and Eulemur fulvus rufus in Madagascar. Am J Primatol 40:327-342

Overdorff DJ, Merenlender AM, Talata P, Telo A, Forward ZA (1999) Life history of Eulemur fulvus rufus from 1988-1998 in Southeastern Madagascar. Am J Phys Anthro 108:295-310

Parrish JK, Edelstein-Keshet $L$ (1999) Complexity, pattern, and evolutionary trade-offs in animal aggregation. Science 284:99-101

Parrish JK, Viscido SV, Grünbaum D (2002) Self-organized fish schools: an examination of emergent properties. Biol Bull 202:296-305

Pays O, Jarman, Loisel P, Gerard JF (2007) Coordination, independence or synchronization of individual vigilance in eastern grey kangaroo? Anim Behav 73:595-604 
Pereira ME (1988) Agonistic interactions of juvenile savanna baboons. I. Fundamental features. Ethology 79:195-217

Pereira ME (1993) Seasonal adjustment of growth rate and adult body weight in ringtailed lemurs. In: Kappeler PM, Ganzhorn JU (eds) Lemur social systems and their ecological basis. Plenum, New York, pp 205-221

Pereira ME, Kappeler PM (1997) Divergent system of agonistic behaviour in lemurid primates. Behaviour 34:225-274

Pereira ME, McGlynn CA (1997) Special relationships instead of female dominance for redfronted lemurs, Eulemur fulvus rufus. Am J Primatol 43:239-258

Pereira ME, Kaufman R, Kappeler PM, Overdorff DJ (1990) Female dominance does not characterize all of the Lemuridae. Folia Primatol 55:96-103

Petit O, Bon R (2010) Decision-making processes: The case of collective movements. Behav Process 84:635-647

Petit O, Gautrais J, Leca JB, Theraulaz G, Deneubourg JL (2009) Collective decision-making in white-faced capuchins. Proc R Soc Lond B 276:3495-3503

Phillips KA, Elvey CR, Abercrombie CL (1998) Applying GPS to the study of primate ecology: a useful tool? Am J Primatol 46:167-172

Pillot MH, Gautrais J, Gouello J, Michelena P, Sibbald A, Bon R (2010) Moving together: Incidental leaders and naïve followers. Behav Process 83:235-241

Pimley ER, Bearder SK, Dixson AF (2005) Home range analysis of Perodicticus potto edwardsi and Sciurocheirus cameronensis. Inter J Primatol 26:191-205

Piyapong C, Morrell L, Croft DP, Dyer JRG, loannou CC, Krause J (2007) A cost of leadership in human groups. Ethology 113:821-824

Pollock IJ (1997) Female dominance in Indri indri. Folia Primatol 31:143-164

Pond CM (1977) The significance of lactation in the evolution of mammmals. Evolution 31:177199

Poole JH, Payne K, Langbauer WR Jr, Moos CJ (1988) The social contexts of some very low frequency calls of African elephants. Behav Ecol Sociobiol 22:385-392

Powell RA (2000) Animal home ranges and territories and home range estimators. In: Boitani L, Fuller TK (eds) Research techniques in animal ecology: Controversies and consequences. Columbia Univ Press, New York, pp 65-110

Prins HHT (1996) Ecology and behaviour of the African buffalo. London, Chapman and Hall

Pulliam HR, Caraco T (1984) Living in groups: is there an optimal group size? In: Krebs JR, Davies MNO (eds) Behavioural ecology. Blackwell, Oxford, pp 122-147

Pyritz L, Andrianjanahary T (2010) Collective mobbing of a boa by a group of red-fronted lemurs (Eulemur fulvus rufus). Lemur News 15:15-17

Pyritz L, Fichtel C, Kappeler P (2010) Conceptual and methodological issues in the comparative study of collective group movements. Behav Process 84:681-684

Pyritz L, King A, Sueur C, Fichtel C (in press) Reaching a consensus: Terminology used in coordination and decision-making research. Int J Primatol 32 (special issue)

Radford AN (2004) Vocal coordination of group movement by green woodhoopoes (Phoeniculus purpureus). Ethology 110:11-20

Rahlfs M, Fichtel C (2010) Anti-predator behaviour in a nocturnal primate, the grey mouse lemur (Microcebus murinus). Ethology 116:429-439

Rakotondravony D, Goodman SM, Soarimalala V (1998) Predation on Hapalemur griseus griseus by Boa manditra (Boidae) in the Littoral Forest of Eastern Madagascar. Folia Primatol 69:405-408

Ramseyer A, Petit O, Thierry B (2009a) Patterns of group movements in juvenile domestic geese. J Ethol 27:369-375

Ramseyer A, Boissy A, Dumont B, Thierry B (2009b) Decision making in group departures of sheep is a continuous process. Anim Behav 78:71-78

Ramseyer A, Thierry B, Boissy A, Dumont B (2009c) Decision-making processes in group departures of cattle. Ethology 115:948-957 
Rands SA, Cowlishaw G, Pettifor RA, Rowcliffe JM, Johnstone RA (2003) Spontaneous emergence of leaders and followers in foraging pairs. Nature 423:432-434

Rands SA, Cowlishaw G, Pettifor RA, Rowcliffe JM, Johnstone RA (2008) The emergence of leaders and followers in foraging pairs when the qualities of individuals differ. BMC Evol Biol 8:51

Rasa OAE (1987) The dwarf mongoose: a study of behaviour and social structure in relation to ecology in a small, social carnivore. Adv Stud Behav 17:121-163

Rasoloarison RM, Rasolonandrasana BPN, Ganzhorn JU, Goodman SM (1995) Predation on vertebrates in the Kirindy forest, western Madagascar. Ecotropica 1:59-65

Rasoloharijaona S, Randrianambinina R, Braune P, Zimmermann E (2006) Loud calling, spacing, and cohesiveness in a nocturnal primate, the Milne Edwards' sportive lemur (Lepilemur edwardsi). Am J Phys Anthropol 129:591-600

Raxworthy CJ (2003) Boidae, Boas. In Goodman SM, Benstead JP (eds) The natural history of Madagascar. Univ of Chicago Press, Chicago, pp 993-997

Reader SM, Laland KN (2002) Social intelligence, innovation, and enhanced brain size in primates. Proc Natl Acad Sci USA 99:4436-4441

Reebs SG (2000) Can a minority of informed leaders determine the foraging movements of a fish shoal? Anim Behav 59:403-409

Reebs SG (2001) Influence of body size on leadership in shoals of Golden Shiners, Notemigonus crysoleucas. Behaviour 138:797-809

Reinhardt V, Reinhardt A, Houser D (1987) Prompted progression order in a troop of captive rhesus monkeys. Folia Primatol 48:121-124

Rempel RS, Rodgers AR, Abraham KF (1995) Performance of a GPS animal location system under boreal forest canopy. J Wildl Manage 59:543-551

Ren B, Li M, Long Y, Grüter CC, Wei F (2008) Measuring daily ranging distances of Rhinopithecus bieti via a global positioning collar at Jinsichang, China: a methodological consideration. Int J Primatol 29:783-794

Rensing S (1999) Immobilization and anesthesia of nonhuman primates. Prim Rep 55:33-38

Richard A (1978) Behavioral Variation: A case study of a Malagasy lemur. Bucknell Univ Press, Lewisburg

Richard AF (1987) Malagasy prosimians: female dominance. In: Smuts BB, Cheney DL, Seyfarth RM, Wrangham RW, Struhsaker TT (eds) Primate societies. Univ of Chicago Press, Chicago, pp 25-33

Rodgers AR, Carr AP (2002) HRE: Home range extension for ArcView. Ontario Ministry of Natural Resources, Centre for Northern Forest Ecosystem Research, Thunder Bay,

Row JR, Blouin-Demers G (2006) Kernels are not accurate estimators of home-range size for herpetofauna. Copeia 4:797-802

Rowell TE (1969) Intra-sexual behavior and female reproductive cycles of baboons (Papio anubis). Anim Behav 17:159-167

Rowell TE (1972) Female reproductive cycles and social behavior in primates. Adv Stud Behav 4:69-105

Rubenstein RI (1994) Ecology of female social behaviour in horses, zebras and asses. In: Jarman PJ, Rossiter A (eds) Individuals, interactions and organizations. Kyoto Univ Press, Kyoto, pp 13-28

Šárová R, Špinka M, Panamá JLA, Šimeček P (2010) Graded leadership by dominant animals in a herd of female beef cattle. Anim Behav 79:1037-1045

Sauther ML, Sussman RW (1993) A new interpretation of the social organization and mating system of the ring-tailed lemur (Lemur catta). In: Kappeler PM, Ganzhorn JU (eds) Lemur social systems and their ecological basis. Plenum, New York, pp 111-121

Scantlebury, M, Russell AF, Mcllrath GM, Speakman JR, Clutton-Brock TH (2002) The energetics of lactation in cooperatively breeding meerkats Suricata suricatta. Proc R Soc Lond B 269:2147-2153 
Scantlebury M, Bennett NC, Speakman JR, Pillay N, Schradin C (2006) Huddling in groups leads to daily energy savings in free-living African four-striped grass mice, Rhabdomys pumilio. Funct Ecol 20:166-173

Schaller G (1963) The mountain gorilla: Ecology and behavior. Univ of Chicago Press, Chicago

Scheumann M, Rabesandratana A, Zimmermann E (2007) Predation, communication, and cognition in lemurs. In: Gursky S, Nekaris KAl (eds) Primate antipredator strategies. Springer, New York, pp 100-126

Schmelting B, Ehresmann P, Lutermann H, Randrianambinina B, Zimmermann E (2000) Reproduction of two sympatric mouse lemur species (Microcebus murinus and $M$. ravelobensis) in north-west Madagascar: first results of a long-term study. In: Lourenço W, Goodman S (eds) Diversité et endémisme à Madagascar. Paris, Société de Biogéographie, pp 165-175

Scholz F, Kappeler PM (2004) Effects of seasonal water scarcity on the ranging behavior of Eulemur fulvus rufus. Int J Primatol 25:599-613

Schülke O (2001) Social anti-predator behaviour in a nocturnal lemur. Folia Primatol 72:332-334

Schuett W, Dall SRX (2009) Sex differences, social context and personality in zebra finches, Taeniopygia guttata. Anim Behav 77:1041-1050

Schulz-Hardt S, Brodbeck FC, Mojzisch A, Kerschreiter R, Frey D (2006) Group decision making in hidden profile situations: dissent as a facilitator for decision quality. J Pers Soc Psychol 91:1080-1091

Seaman DE, Powell RA (1996) An evaluation of the accuracy of kernel density estimators for home range analysis. Ecology 77:2075-2085

Seaman DE, Millspaugh JJ, Kernohan BJ, Brundige GC, Raedeke KJ, Gitzen RA (1999) Effects of sample size on kernel home range estimates. J Wildl Manage 63:739-747

Seeley TD, Buhrman SC (2001) Nest-site selection in honey bees: how well do swarms implement the 'best-of- $N$ ' decision rule? Behav Ecol Sociobiol 49:416-427

Seeley TD, Visscher PK (2004) Quorum sensing during nest-site selection by honeybee swarms. Behav Ecol Sociobiol 56:594-601

Seeley TD, Visscher PK, Passino KM (2006) Group decision making in honey bee swarms. Am Sci 94:220-229

Sellers WI, Hill RA, Logan BS (2007) An agent-based model of group decision making in baboons. Phil Trans R Soc Lond B 362:1699-1710

Seyfarth RM (1986) Vocal communication and its relation to language. In: Smuts BB, Cheney DL, Seyfarth RM, Wrangham RW, Struhsaker TT (eds) Primate societies. Chicago Univ Press, Chicago, pp 440-451

Shields W (1984) Barn swallow mobbing: self-defence, collateral kin defence, group defence, or parental care? Anim Behav 32:132-148

Shultz S, Dunbar RIM (2007) The evolution of the social brain: Anthropoid primates contrast with other vertebrates. Proc R Soc Lond B 274:2429-2436

Sigg H, Stolba A (1981) Home range and daily march in a hamadryas baboon troop. Folia Primatol 36:40-75

Silk JB, Alberts SC, Altmann J (2003) Social bonds of female baboons enhance infant survival. Science 302:1231-1234

Simmen B, Bayart F, Rasamimanana H, Zahariev A, Blanc S, Pasquet P (2010) Total energy expenditure and body composition in two free-living sympatric lemurs. PLoS ONE 5: e9860. doi:10.1371/journal.pone.0009860

Sirot $E$, Touzalin $F$ (2009) Coordination and synchronization of vigilance in groups of prey: the role of collective detection and predators' preference for stragglers. Am Nat 173:47-59

Slip D, Shine R (1988) Feeding habits of the diamond python, Morelia s. spilota: ambush predation by a Boid snake. J Herpetol 22:323-330

Smuts BB, Cheney DL, Seyfarth RM, Wrangham RW, Struhsaker TT (1986) Primate societies. Univ of Chicago Press, Chicago 
Soini P (1981) The pygmy marmoset, genus Cebuella. In: Mittermeier RA, Rylands AB, CoimbraFilho AF (eds) Ecology and behavior of Neotropical primates. Academia Brasileira de Ciencias, Rio de Janeiro, pp 79-129

Sorg JP, Rohner U (1996) Climate and tree phenology of the dry deciduous forest of the Kirindy Forest. In: Ganzhorn, Sorg JP (eds) Ecology and economy of a tropical dry forest in Madagascar. Erich Goltze, Göttingen, pp. 57-80

Sorg JP, Ganzhorn JU, Kappeler PM (2003) Forestry and research in the Kirindy Forest/Centre de Formation Professionnelle Forestière. In: Goodman SM, Benstead JP (eds) The natural history of Madagascar. Univ of Chicago Press, Chicago, pp 1512-1519

Spong G (2002) Space use in lions, Panthera leo, in the Selous Game Reserve: social and ecological factors. Behav Ecol Sociobiol 52:303-307

Sprague DS, Kabaya H, Hagihara K (2004) Field testing a global system (GPS) collar on a Japanese monkey: Reliability of automatic GPS positioning in a Japanese forest. Primates 45:151154

Stanford CB (1990) Colobine socioecology and female-bonded models of primate social structure. Kroeber Anthropol Soc Pap 71-72:21-28

Sterck EHM, Watts DP, van Schaik CP (1997) The evolution of female social relationships in nonhuman primates. Behav Ecol Sociobiol 41:291-309

Stewart KJ, Harcourt AH (1994) Gorillas' vocalisations during rest periods: signals of impending departure? Behaviour 130:29-40

Stolba A (1979) Entscheidungsfindung in Verbänden von Papio hamadryas (in German). Dissertation. Univ of Zurich, Zurich

Struhsaker TT (1967a) Auditory communication among vervet monkeys (Cercopithecus aethiops). In: Altmann SA (ed) Social communication among primates. Univ of Chicago Press, Chicago, pp 281-324

Struhsaker TT (1967b) Ecology of vervet monkeys (Cercopithecus aethiops) in the Masai-Amboseli Game Reserve, Kenya. Ecology 48:891-904

Struhsaker TT (1975) The red colobus monkey. Univ of Chicago Press, Chicago

Stueckle S, Zinner D (2008) To follow or not to follow: decision making and leadership during the morning departure in chacma baboons. Anim Behav 75:1995-2004

Sueur C, Petit O (2008a) Shared or unshared consensus decision in macaques? Behav Process 78:84-92

Sueur C, Petit O (2008b) Organization of group members at departure is driven by social structure in Macaca. Int J Primatol 29:1085-1098

Sueur C, Petit O (2010) Signals use by leaders in Macaca tonkeana and Macaca mulatta: groupmate recruitment and behaviour monitoring. Anim Cog 13:239-248

Sueur C, Deneubourg JL (2011) Self-organization in primates: Understanding the rules underlying collective movements. Int J Primatol, doi:10.1007/s10764-011-9520-0

Sueur C, Petit O, Deneubourg JL (2009) Selective mimetism at departure in collective movements of Macaca tonkeana: an experimental and theoretical combination of mimetic rules in macaques. Anim Behav 78:1087-1095

Sueur C, Deneubourg JL, Petit O (2010) From the first intention movement to the last joiner: macaques combine mimetic rules to optimize their collective decisions. Proc R Soc Lond B, doi:10.1098/rspb.2010.2084

Sumpter DJT (2006) The principles of collective animal behaviour. Philos Trans R Soc Lond B 361:522

Sussman RW (1974) Ecological distinctions of sympatric species of Lemur. In: Martin RD, Doyle GA, Walker AC (eds.) Prosimian Biology. Duckworth, London

Sussman RW (1975) A preliminary study of the behavior and ecology of Lemur fulvus rufus Audebert 1800. In: Tattersall I, Sussman RW (eds) Lemur biology. Plenum Press, New York, pp 237-258

Sutherland WJ (1998) The importance of behavioural studies in conservation biology. Anim Behav 56:801-809 
Swihart RK, Slade NA (1985) Influence of sampling interval on estimates of home-range size. J Wildl Manage 49:1019-1025

Swihart RK, Slade NA (1997) On testing for independence of animal movements. J Agric Biol Envir S 2:48-63

Takenoshita Y, Sprague D, Iwasaki N (2004) Factors affecting success rate and accuracy of GPS collar positioning for free-ranging Japanese macaques. Prim Res 21:107-119

Tamura N (1989) Snake-directed mobbing by the Formosan squirrel Callosciurus erythraeus thaiwanensis. Behav Ecol Sociobiol 24:175-180

Tarnaud L (2006) Feeding behavior of lactating brown lemur females (Eulemur fulvus) in Mayotte: Influence of infant age and plant phenology. Am J Primatol 68:966-977

Tello NS, Huck M, Heymann EW (2002) Boa constrictor attack and successful group defence in moustached tamarins, Saguinus mystax. Folia Primatol 73:146-148

Tomasello M, Zuberbüler K (2002) Primate vocal and gestural communication. In: Bekoff M, Allen $C$, Burghardt $\mathrm{G}$ (eds) The cognitive animal: Empirical and theoretical perspecitives on animal cognition. MIT Press, Cambridge, Massachusetts

Tomkiewicz, S. M., Fuller, M. R., Kie, J. G., \& Bates, K. K. (2010). Global positioning system and associated technologies in animal behaviour and ecological research. Phil Trans $\mathrm{R}$ Soc Lond B 365:2163-2176

Trillmich J, Fichtel C, Kappeler PM (2004) Coordination of group movements in wild Verreaux`s sifakas (Propithecus verreauxi). Behaviour 141:1103-1120

van Nordwijk MA, van Schaik CP (1987) Competition among female long-tailed macaques, Macaca fascicularis. Anim Behav 35:577-589

van Nordwijk MA, Hemelrijk C, Herremans L, Sterck E (1993) Spatial position and behavioral sex differences in juvenile long-tailed macaques. In: Pereira $M$, Fairbanks $L$ (eds) Juvenile primates. Oxford Univ Press, New York, pp 77-85

van Schaik C (1983) Why are diurnal primates living in groups? Behaviour 88:120-143

van Schaik CP (1989) The ecology of social relationships amongst female primates. In: Standen V, Foley RA (eds) Comparative socioecology. The behavioral ecology of humans and other mammals. Blackwell, Oxford, pp 195-218

van Schaik CP, van Noordwijk MA (1985) Evolutionary effect of the absence of felids on the social organization of the macaques on the island of Simeulue (Macaca fascicularis, Miller 1903). Folia Primatol 44:138-147

Van Vugt M (2006) Evolutionary origins of leadership and followership. Pers Soc Psychol Rev 10:354-371

Vogel C (1973) Acoustical communication among free-ranging common Indian langurs (Presbytis entellus) in two different habitats of North India. Am J Phys Anthropol 38:469-480

Vogel ER (2005) Rank differences in energy intake rates in white-faced capuchin monkeys, Cebus capucinus: the effects of contest competition. Behav Ecol Sociobiol 58:333-344

Ward AJW, Thomas P, Hart PJB, Krause J (2004) Correlates of boldness in three-spined sticklebacks (Gasterosteus aculeatus). Behav Ecol Sociobiol 55:561-568

Ward AJW, Sumpter DJT, Couzin ID, Hart PJB, Krause J (2008) Quorum decision-making facilitates information transfer in fish shoals. Proc Natl Acad Sci USA: 105:6948-6953

Ward AJW, Herbert-Read JE, Sumpter DJT, Krause J (2011) Fast and accurate decisions through collective vigilance in fish shoals. Proc Natl Acad Sci USA 108:2312-2315

Warren RD, Crompton RH (1997) A comparative study of the ranging behaviour, activity rhythms and sociality of Lepilemur edwardsi (Primates, Lepilemuridae) and Avahi occidentalis (Primates, Indriidae) at Ampijora, Madagascar. J Zool 243:397-415

Watts DP (1994) The influence of male mating tactics on habitat use in mountain gorillas (Gorilla gorilla berengei). Primates 35:35-47

Wheeler BC (2009) Monkeys crying wolf? Tufted capuchin monkeys use anti-predator calls to usurp resources from conspecifics. Proc R Soc B 276: 3013-3018

White G, Garrott R (1990) Analysis of wildlife radio tracking data. Academic, San Diego 
Whitehead JM (1989) The effect of the location of a simulated intruder on responses to long distance vocalizations of mantled howling monkeys, Alouatta palliata palliata. Anim Behav 108:73-103

Williams SC, DeNicola AJ, Ortega IM (2008) Behavioral responses of white-tailed deer subjected to lethal management. Can J Zoolog 86:1358-1366

Wimmer B, Kappeler PM (2002) The effects of sexual selection and life history on the genetic structure of redfronted lemur, Eulemur fulvus rufus, groups. Anim Behav 64:557-68

Wolfheim JH (1983) Primates of the world: Distribution, abundance and conservation. Univ of Washington Press, Seattle

Worton BJ (1989) Kernel methods for estimating the utilization distribution in home-range studies. Ecology 70:164-168

Worton BJ (1995a) Using Monte Carlo simulation to evaluate kernel-based home range estimators. J Wildl Manage 59:794-800

Worton BJ (1995b) A convex hull-based estimator of home range size. Biometrics 51:1206-1215

Wrangham RW (1980) An ecological model of female-bonded primate groups. Behaviour 75:262300

Wrangham RW (2000) Why are male chimpanzees more gregarious than mothers? A scramble competition hypothesis. In: Kappeler PM (ed) Primate males. Causes and consequences of variation in group composition. Cambridge Univ Press, Cambridge, pp 248-258

Wright J, Stone RE, Brown N (2003) Communal roosts as structured information centres in the raven, Corvus corax. J Anim Ecol 72:1003-101

Zeller AC (1986) Communication by sight and smell. In: Smuts BB, Cheney DL, Seyfarth RM, Wrangham RW, Struhsaker TT (eds) Primate societies. Univ of Chicago Press, Chicago, pp 433-439

Zemel A, Lubin Y (1995) Inter-group competition and stable group sizes. Anim Behav 50:485-488

Zinner D, Hilgartner RD, Kappeler PM, Pietsch T, Ganzhorn JU (2003) Social organization of Lepilemur ruficaudatus. Int J Primatol 24:869-888

Zuur AF, leno EN, Walker NJ, Saveliev AA, Smith GM (2009) Mixed effects models and extensions in ecology with R. Springer, New York 


\section{ACKNOWLEDGEMENTS}

I would like to thank my supervisor Peter Kappeler for giving me the opportunity to explore something as fascinating and exotic as group coordination in lemurs on the even more exotic island of Madagascar. I am grateful for his support and constructive discussions in every stage of this project and for the opportunity to be part of an inspiring scientific environment. I am also particularly grateful to Claudia Fichtel who co-supervised this work from beginning to end: Thank you for the frequent discussions, scientific guidance and dedicated support throughout the thesis. I also thank Eckhard Heymann for his effort and support as a referee of this dissertation and for giving me valuable advice on my diploma thesis that brought me into contact with the German Primate Center (DPZ) in the first place.

The field work in Madagascar would not have been possible without the support of the great "Equipe Kirindy" including all field assistants, the kitchen staff and Samy`s familiy in Morondava. Isaorako eram-po ireo mpiara-miasa tao Kirindy noho ny fifanampiana tamin'ny ny asa rehetra natao tany Madagasikara, ny hirahira sy kalo niarahana, ny tsikitsiky sy hehy nifampizàrana, ny fiarahana nahafinaritra. Tsy hanadino anareo aho.

I am particularly grateful to Jean-Pierre Tolojanahary who collected a large proportion of the data presented in this thesis, and who was the best companion I can imagine during the long forest hours that we spent together. Furthermore, I thank Rémy d’Ampataka, Tianasoa Andrianjanahary, Patrick de Beroboka, Rodin Rasoloarison and Léonard Razafimanantsoa for assistance in the field, logistic support and splendid evenings at Sam Suffy's. I also thank the following Malagasy institutions for their kind authorization and support of this study: Département de Biologie Animale, Université d'Antananarivo; the CAFF of the Direction des Eaux et Forêts and the CNFEREF Morondava.

For the great times in Germany, Madagascar and during conferences throughout the last years I am grateful to all my colleagues from the DPZ unit and University department. In particular, I would like to thank Dagmar Clough, Melanie Dammhahn, Elise Huchard, Conny Kraus, Yvan Lledo-Ferrer, Mia-Lana Lührs, Matthias Markolf, Femke Pflüger, Markus Port, Moritz Rahlfs and Susanne Schliehe-Diecks for their support. I would 
also like to thank friends and colleagues from other departments of the DPZ and the Courant Research Centre "Evolution of Social Behaviour" as well as Jörg Ganzhorn, Andrew King, Charles Nunn and Cédric Sueur for inspiring discussions on group coordination. Greg Bubnis, Michael Hellriegel, Stephanie Plehm and Moritz Rahlfs provided valuable comments on earlier versions of this manuscript and polished the English in some parts.

I am thankful for the logistic support provided by Anja Engelke, Christina Oberdieck, Ulrike Walbaum and Guni Wilz. For excellent technical support I thank Manfred Eberle, Franz Kümmeth (e-obs GmbH Grünwald, München), Henning Lahmann as well as Lothar Neumann and his team in Ettlingen.

The German Research Foundation (DFG: KA 1082/16-1) and the German Academic Exchange Service (DAAD) kindly provided financial support. I am also grateful to Joanna Setchell and the International Journal of Primatology for funds that contributed towards the travel costs to the XXIII Congress of the International Primatological Society (IPS) in Japan and for supporting the special issue on group coordination and decision-making.

I am deeply thankful to my parents, family and friends for their support, the zoological enthusiasm of the "Swedish Lemur League" and the care packages and encouraging mails and letters I received in Madagascar. At this point, I would also like to thank Mr Wilm who was my biology teacher back in high school for promoting my fascination for biology and for the postcards he sent to me from his various ornithological excursions all over the world after his retirement.

Finally: Thank you, Anike, for staying by my side even during the long times we were on different continents - and for everything else. 


\section{APPENDIX}

Table A1 (Chapter 4): Number of visits on single platforms per study day throughout different conditions/designs.

\begin{tabular}{|c|c|c|c|c|c|c|}
\hline \multirow[t]{2}{*}{ Design } & \multirow[t]{2}{*}{ Condition } & \multirow{2}{*}{$\begin{array}{l}\text { Day of } \\
\text { experiment }\end{array}$} & \multicolumn{4}{|c|}{ Platform number } \\
\hline & & & 1 & 2 & 3 & 4 \\
\hline \multirow[t]{4}{*}{0} & \multirow[t]{4}{*}{1} & 1 & 4 & 3 & 4 & 0 \\
\hline & & 2 & 0 & 1 & 0 & 0 \\
\hline & & 3 & 1 & 1 & 3 & 0 \\
\hline & & 4 & 0 & 0 & 2 & 0 \\
\hline \multirow[t]{8}{*}{1} & \multirow[t]{4}{*}{2} & 5 & 1 & 1 & 2 & 1 \\
\hline & & 6 & 2 & 0 & 1 & 0 \\
\hline & & 7 & 2 & 1 & 3 & 0 \\
\hline & & 8 & 2 & 1 & 2 & 1 \\
\hline & \multirow[t]{4}{*}{3} & 9 & 0 & 1 & 3 & 0 \\
\hline & & 10 & 2 & 1 & 4 & 3 \\
\hline & & 11 & 2 & 1 & 3 & 4 \\
\hline & & 12 & 1 & 1 & 1 & 1 \\
\hline \multirow[t]{8}{*}{2} & \multirow[t]{4}{*}{4} & 13 & 0 & 1 & 2 & 2 \\
\hline & & 14 & 2 & 3 & 2 & 0 \\
\hline & & 15 & 0 & 1 & 1 & 1 \\
\hline & & 16 & 0 & 1 & 3 & 0 \\
\hline & \multirow[t]{4}{*}{5} & 17 & 1 & 3 & 2 & 0 \\
\hline & & 18 & 1 & 0 & 4 & 1 \\
\hline & & 19 & 0 & 3 & 4 & 2 \\
\hline & & 20 & 0 & 1 & 2 & 0 \\
\hline
\end{tabular}




\section{CURRICULUM VITAE}

\section{Personal information}

\begin{tabular}{ll}
\hline Name & Lennart Wolfgang Pyritz \\
Date of birth & April 20,1979 \\
Place of birth & Bielefeld \\
Citizenship & German
\end{tabular}

\section{Education}

since 2009 Member of the Courant Research Centre Evolution of Social Behaviour

since 2007 Ph.D. project, Behavioral Ecology and Sociobiology Unit, German Primate Center (supervised by Dr. Claudia Fichtel, Prof. Dr. Peter Kappeler and Prof. Dr. Eckhard Heymann)

M.Sc. (Dipl.-Biol.), University of Göttingen, Germany

Thesis (supervised by PD Dr. Michael Kessler): "Effects of forest fragmentation on diversity and abundance of birds and primates in tropical deciduous forests in Santa Cruz, Bolivia"

2000 - 2006 Studies in Biology, University of Göttingen, Germany

$1999-2000$ Studies in Philosophy, University of Bielefeld, Germany

1998

Abitur, Gymnasium Verl, Germany

\section{Work experience}

\begin{tabular}{ll}
\hline $2007-2010$ & $\begin{array}{l}\text { 14 months of field work, Kirindy Research Station, Madagascar } \\
2006-2007\end{array}$ \\
$\begin{array}{l}\text { Research assistant, Institute of Zoology and Anthropology, } \\
\text { University of Göttingen, Germany }\end{array}$ \\
$2003-2004 \quad \begin{array}{l}\text { Practical semester in Santa Cruz, Bolivia: Research assistant in } \\
\text { ornithological field study at Los Volcanes Research Station }\end{array}$ \\
$2001-2005$ & $\begin{array}{l}\text { Member of the Primate Working Group, Cologne Zoo, Germany } \\
2000-2001\end{array}$ \\
& $\begin{array}{l}\text { Research assistant, Ethologische Station Sennickerode, } \\
\text { University of Göttingen, Germany }\end{array}$
\end{tabular}

